\title{
METHODOLOGY FOR COLLISION RISK ASSESSMENT OF AN AIRSPACE FLOW CORRIDOR CONCEPT
}

by

Yimin Zhang:

A Dissertation

Submitted to the

Graduate Faculty of

George Mason University

in Partial Fulfillment of

The Requirements for the Degree

of

Doctor of Philosophy

Systems Engineering and Operations Research

Committee:

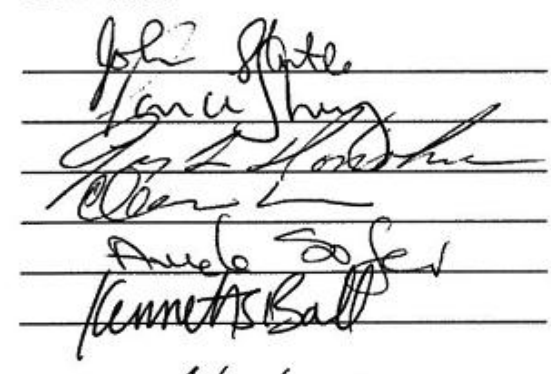

Date: $4 / 30 / 2014$

Dr. John Shortle, Dissertation Director

Dr. Lance Sherry, Dissertation Co-director

Dr. George Donohue, Committee Member

Dr. Chi-Hyon Lee, Committee Member

Dr. Ariela Sofer, Department Chair

Dr.Kenneth S. Ball, Dean, Volgenau School of Engineering

Spring Semester 2014

George Mason University

Fairfax, VA 
Methodology for Collision Risk Assessment of an Airspace Flow Corridor Concept

A Dissertation submitted in partial fulfillment of the requirements for the degree of Doctor of Philosophy at George Mason University

$$
\text { by }
$$

\author{
Yimin Zhang \\ Bachelor of Since \\ Harbin Engineering University, 2007
}

Director: John Shortle, Professor

Department of Systems Engineering and Operations Research

Spring Semester 2014

George Mason University

Fairfax, VA 


\section{(c) (i) $=$}

This work is licensed under a creative commons attribution-noderivs 3.0 unported license. 


\section{ACKNOWLEDGEMENTS}

I would like to thank the many friends, relatives, and supporters who have made this happen. My loving wife, Yawen, supported me all the way in my research. Drs. Shortle, Sherry, Donohue and Lee were of invaluable help. Finally, thanks go out to the Fenwick Library for providing a clean, quiet, and well-equipped repository in which to work.

This work was sponsored in part by NASA Ames Research Center (NRA number NNH08ZEA001N). The opinions and results in this paper are solely those of the author. 


\section{TABLE OF CONTENTS}

Page

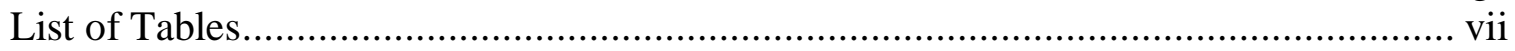

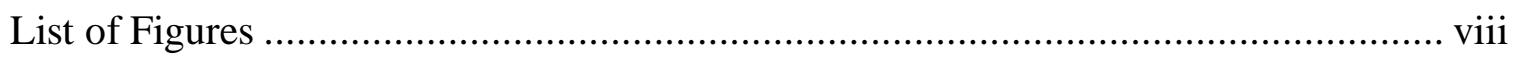

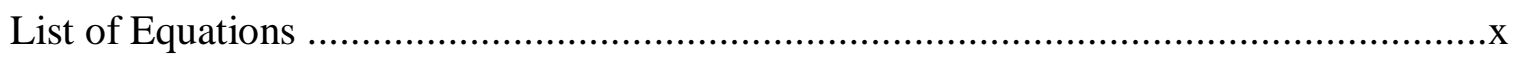

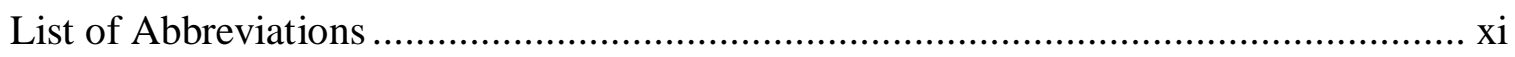

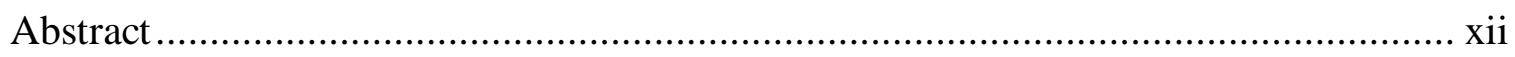

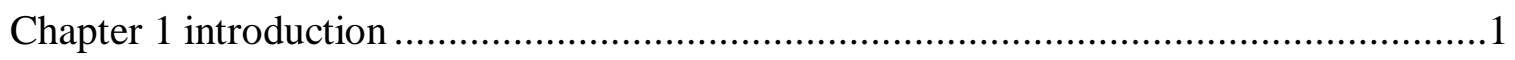

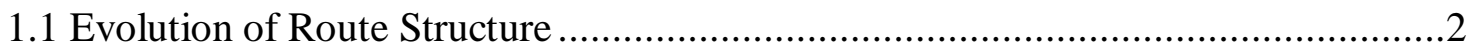

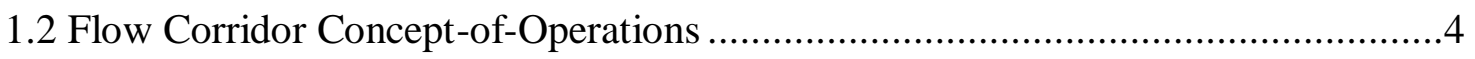

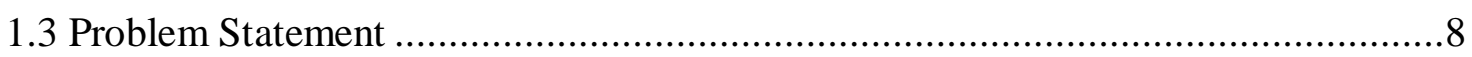

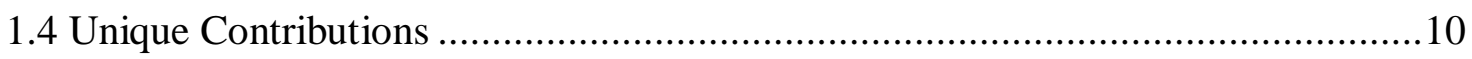

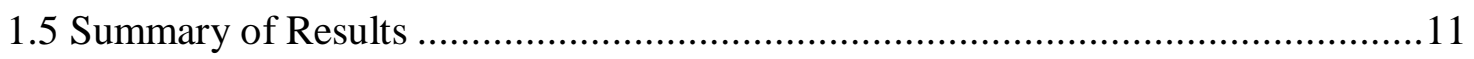

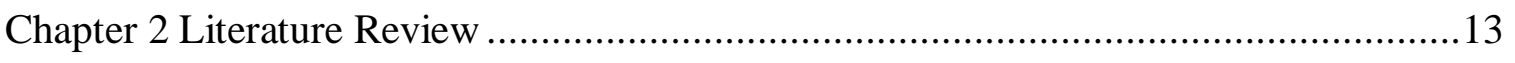

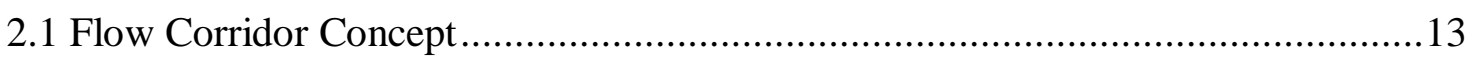

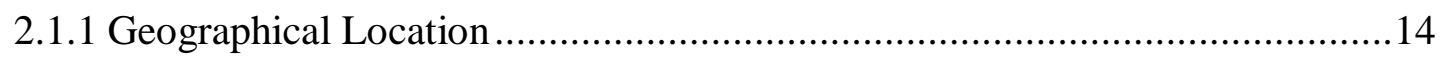

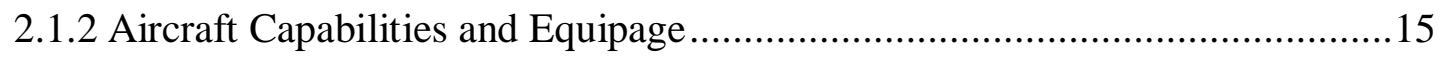

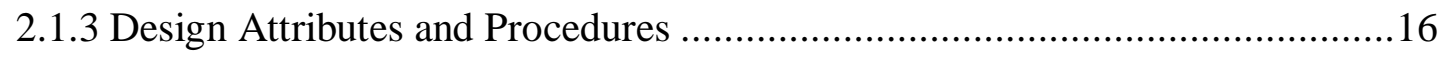

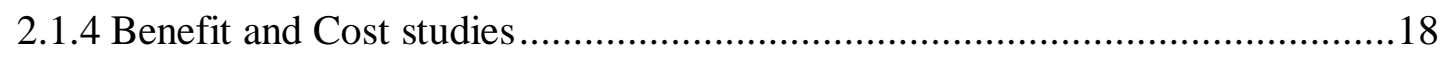

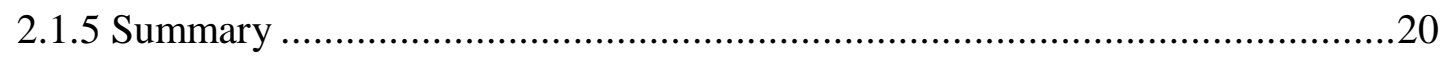

2.2 Collision Risk Models for Parallel Routes …………......................................20

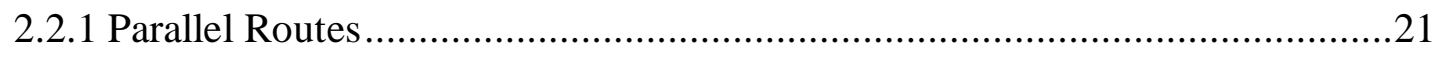

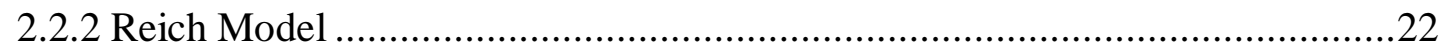

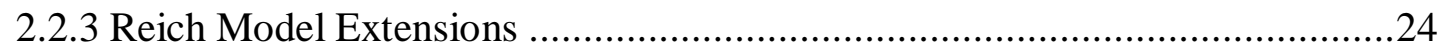

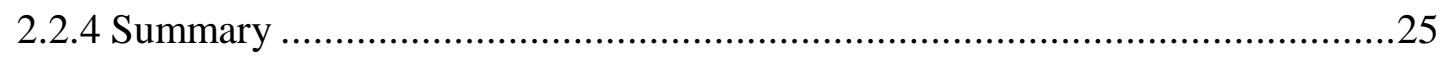

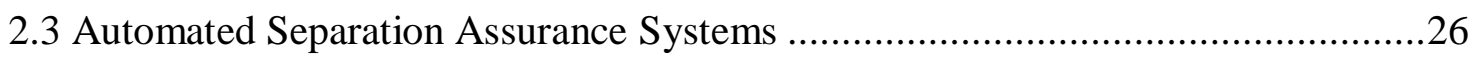

2.3.1 Autonomous Flight Management Concept ................................................26

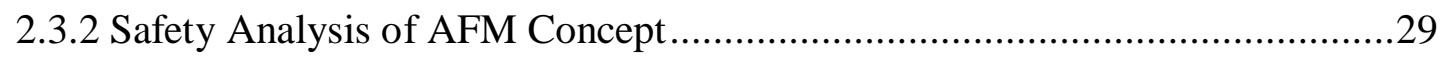


2.3.3 Conflict Detection and Resolution Algorithms ..............................................

2.4 Traffic Collision Avoidance System .................................................................

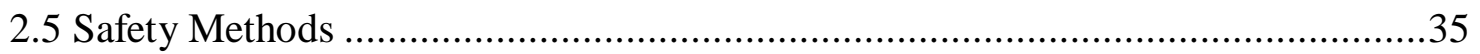

Chapter 3 Methodology for Collision probability estimation of flow corridors................38

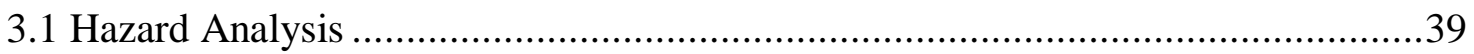

3.2 Methodology and Framework to Estimate Collision Probability ...........................42

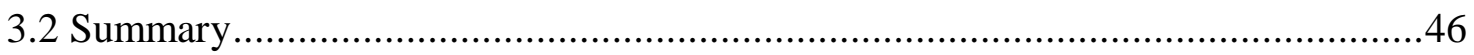

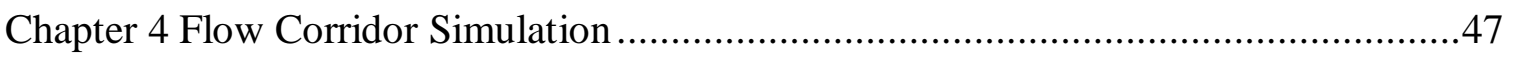

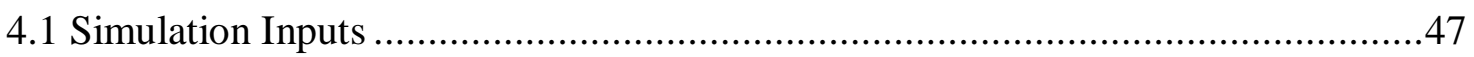

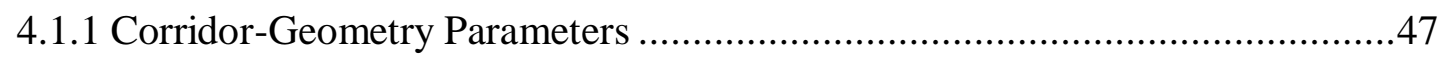

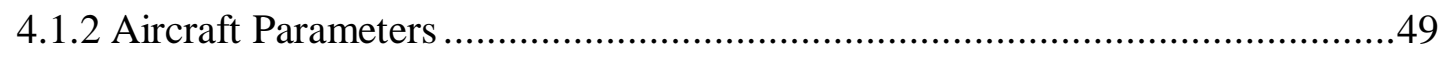

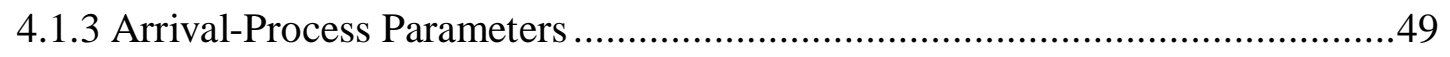

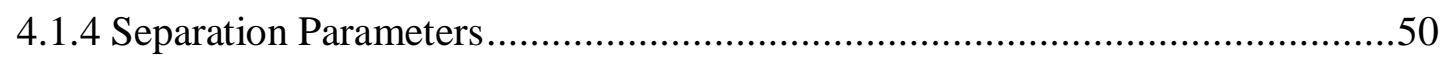

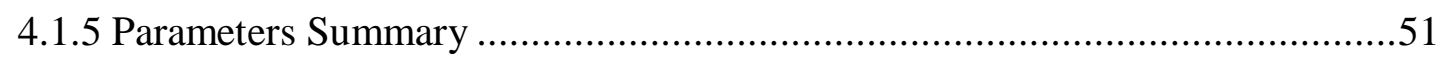

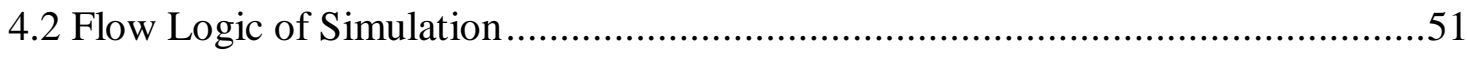

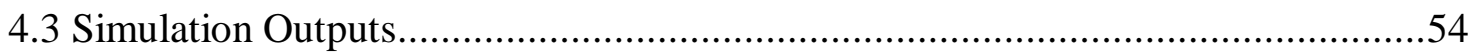

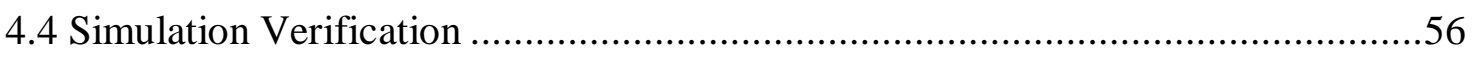

Chapter 5 Dynamic event tree and fault tree analysis ................................................60

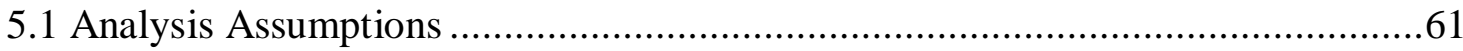

5.2 Events Leading to Actual NMAC Trajectory ……............................................62

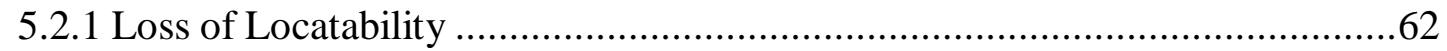

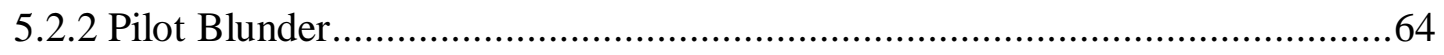

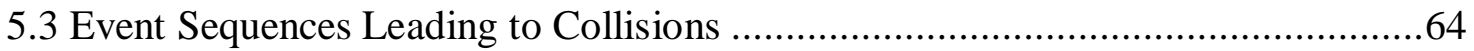

5.4 Difference between Events Sequence Leading to Collisions ..................................71

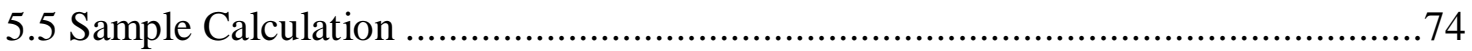

Chapter 6 RESULTS FOR SIMULATION AND DYNAMIC EVENT TREE

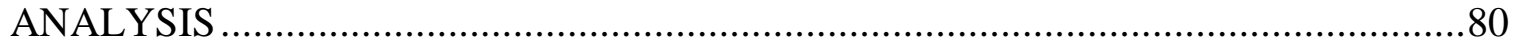

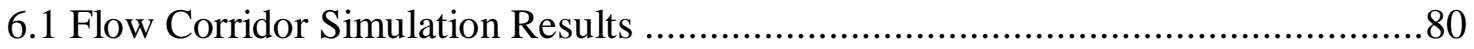

6.2 Results of Dynamic Event Tree Analysis .........................................................94

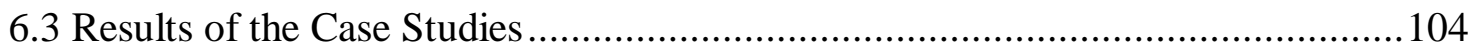

6.4 Trade-off between Throughput and Collision Probability ....................................106

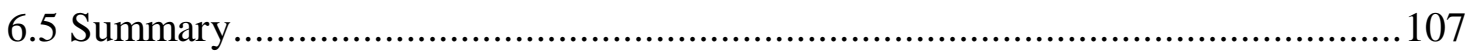

Chapter 7: Conclusions and Recommendations for Future Research ...........................109 


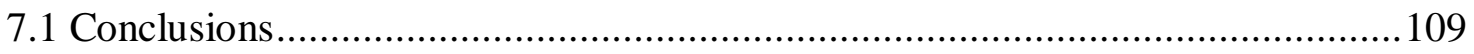

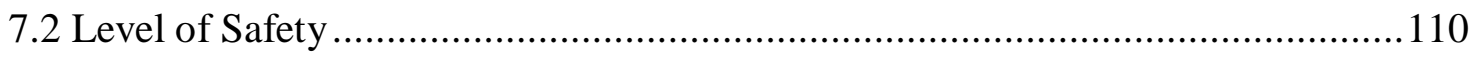

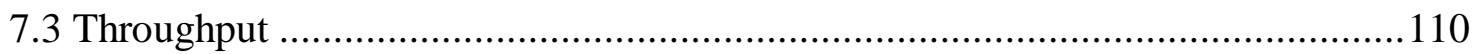

7.4 Key Safety Vulnerabilities ........................................................................ 110

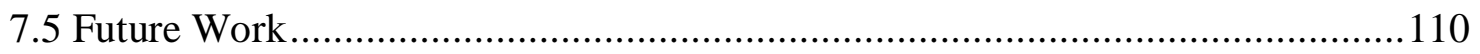

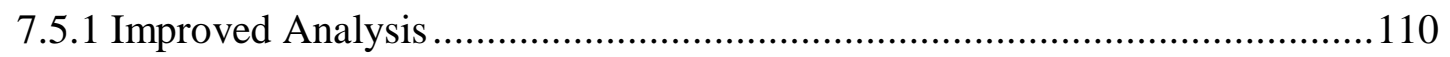

7.5.2 Improved Modeling ..................................................................... 110

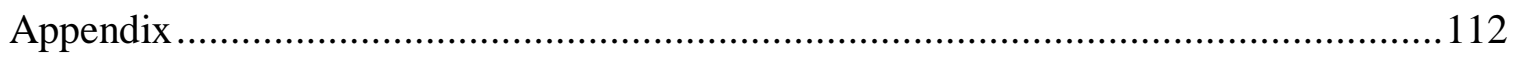

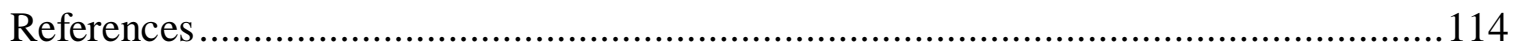




\section{LIST OF TABLES}

Table $\quad$ Page

Table 1 En-route delays with 2X demand [FAA 2007] ...............................................

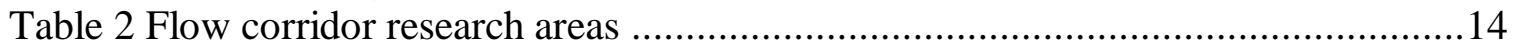

Table 3 Corridor design attributes and alternatives [Wing et al. 2008] .........................17

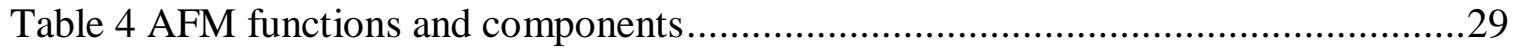

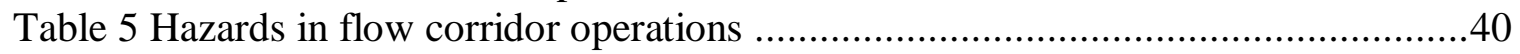

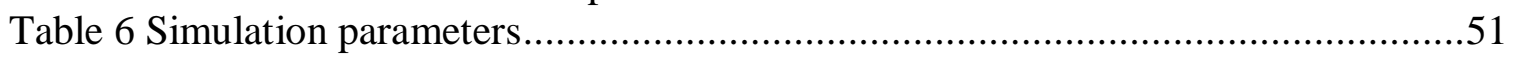

Table 7 Starting functions for collision scenarios ...................................................... 72

Table 8 Relationship between reliability diagrams and event trees, for one aircraft $(1=$

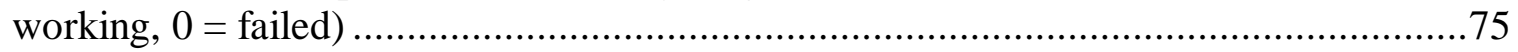

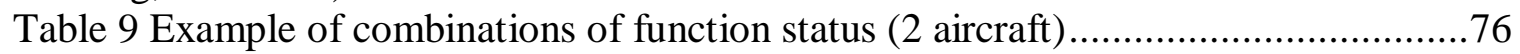

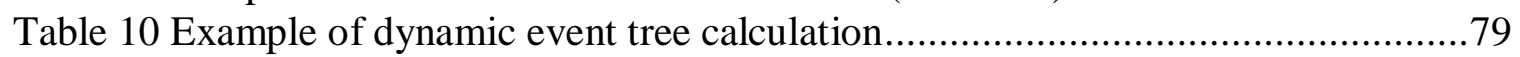

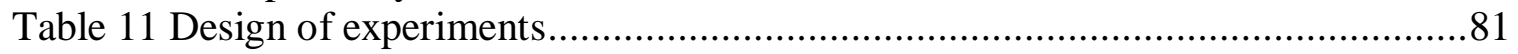

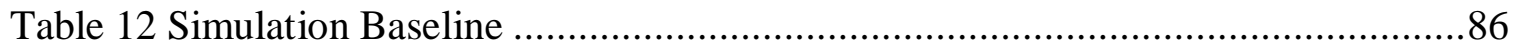

Table 13 Conditional collision probability of overtaking scenario due to pilot blunder...96 Table 14 Conditional collision probability of lane change scenario due to pilot blunder 98 Table 15 Conditional collision probability of simultaneous lane change scenario due to pilot blunder

Table 16 Conditional collision probability of lane change and overtaking scenario due to

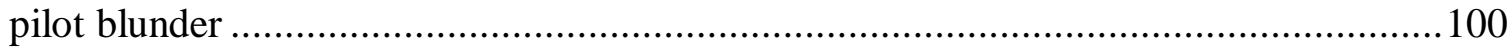

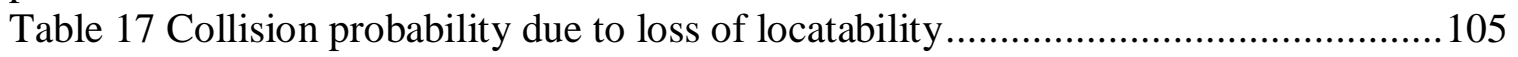

Table 18 Collision probability duel to pilot blunders ........................................... 105

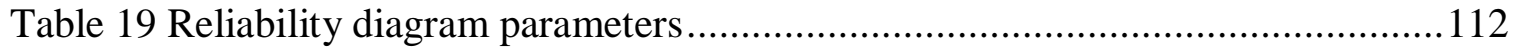

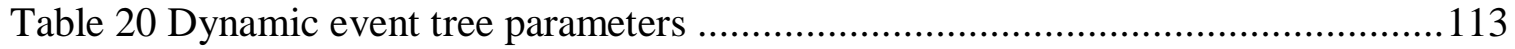




\section{LIST OF FIGURES}

Figure $\quad$ Page

Figure 1 Evolution of route structure .....................................................................

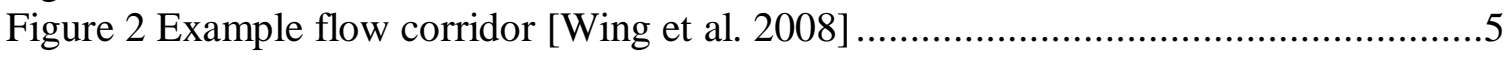

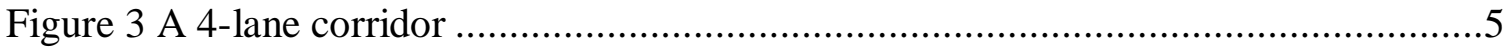

Figure 4 Relation between self separation and automated separation assurance system ....8

Figure 5 Flights comparison during peak hour for 2X demand [Wing et al. 2008] .........19

Figure 6 NAS wide delay reduction with length of flow corridors [Yousefi et al. 2010].20

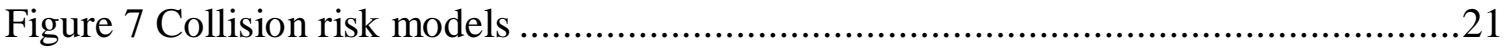

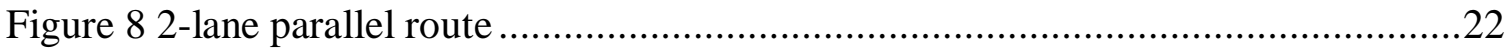

Figure 9 AFM concept diagram [Wing and Ballin 2004] .......................................28

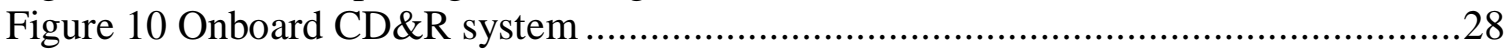

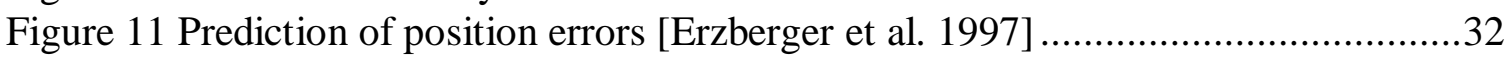

Figure 12 Pre-cursor scenarios to collision..............................................................43

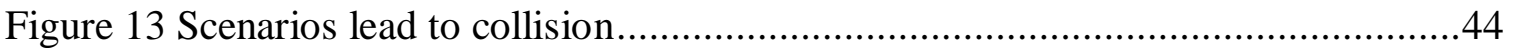

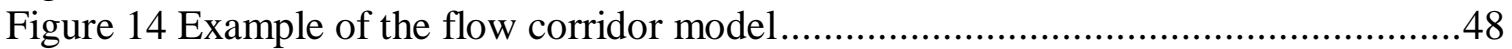

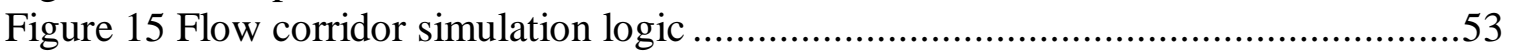

Figure 16 Example of lane change maneuver .....................................................54

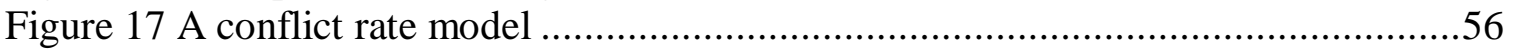

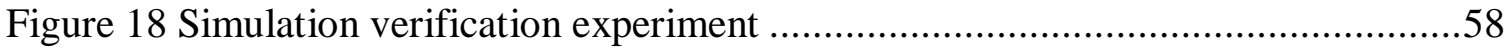

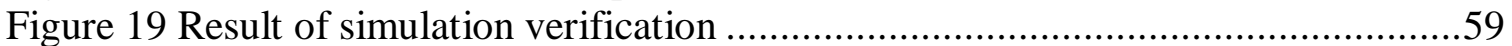

Figure 20 Reliability diagram of locatability function .........................................63

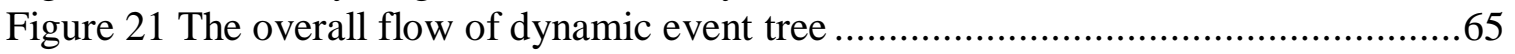

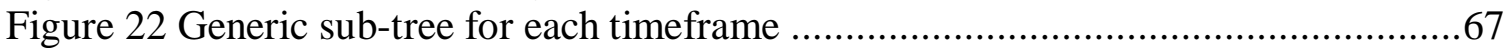

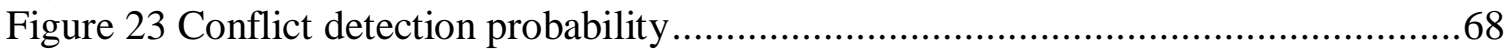

Figure 24 Reliability diagram of strategic intent-based function.................................69

Figure 25 Reliability diagram of tactical intent-based function.................................... 70

Figure 26 Reliability diagram of tactical state-based function ....................................70

Figure 27 Reliability diagram of TCAS function................................................... 71

Figure 28 A simple calculation to estimate the starting point of lane change maneuver ..72

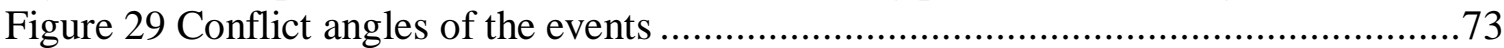

Figure 30 Experiment of varying minimum separation............................................ 82

Figure 31 Experiment of varying fleet mix.......................................................... 83

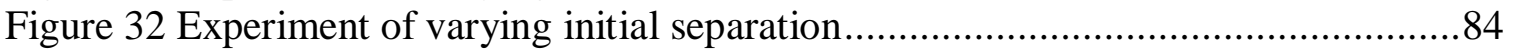

Figure 33 Experiment of varying standard deviation of target speed ...........................85

Figure 34 Experiment of varying relative speed threshold ......................................... 86

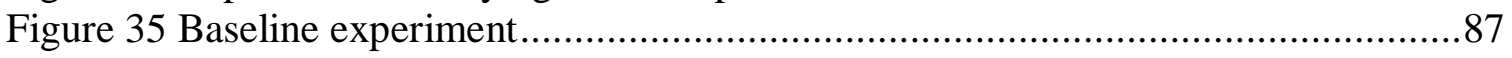


Figure 36 Full factorial analysis

Figure 37 Results of full factorial analysis for collision scenarios (increasing parameter effect on potential NMAC trajectory rate).

Figure 38 Results of full factorial analysis for overtaking scenario (increasing parameter effect on potential NMAC trajectory rate)

Figure 39 Results of full factorial analysis for lane change scenario (increasing parameter

effect on potential NMAC trajectory rate).

Figure 40 Results of full factorial analysis for simultaneous lane change scenario (increasing parameter effect on potential NMAC trajectory rate)

Figure 41 Results of full factorial analysis for simultaneous and lane change scenario

(increasing parameter effect on potential NMAC trajectory rate) ................................94

Figure 42 Failure probabilities of states of functions ..............................................97

Figure 43 Sensitivity analysis of collision scenarios due to loss of locatability ............101

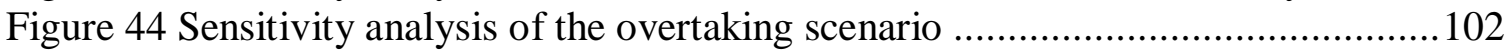

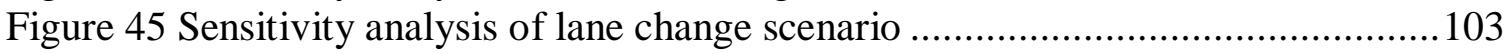

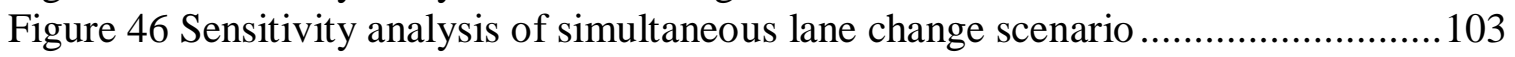

Figure 47 Sensitivity analysis of lane change and overtaking scenario ....................... 104

Figure 48 Trade-off between throughput and collision probability ............................ 107 


\section{LIST OF EQUATIONS}

Equation Page

Equation 1 Expected lateral collisions per flight hour ..............................................23

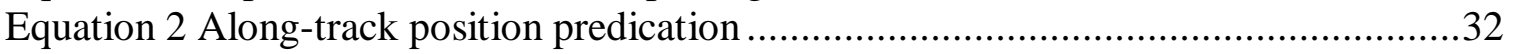

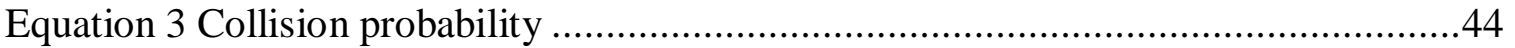

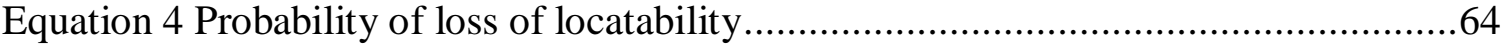




\section{LIST OF ABBREVIATIONS}

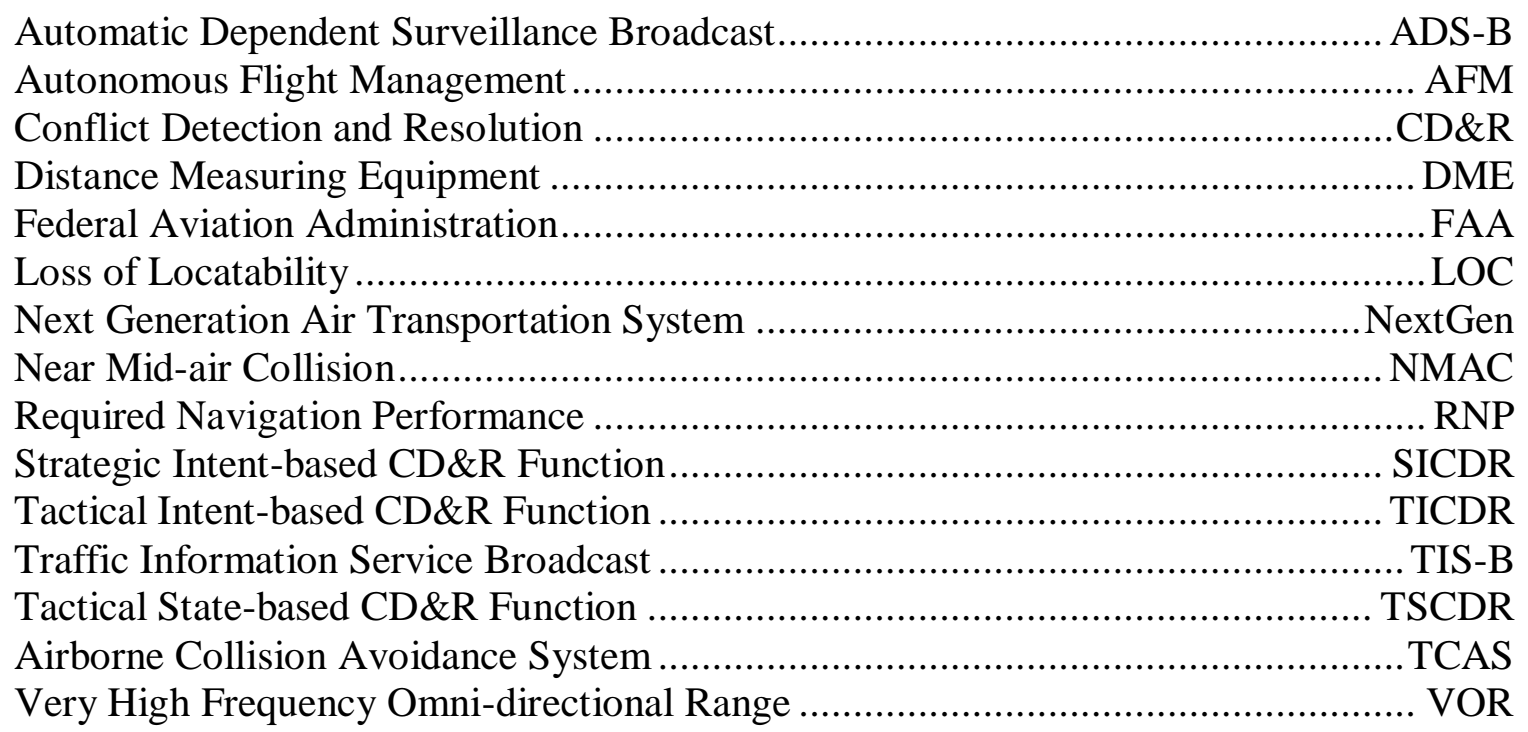




\begin{abstract}
METHODOLOGY FOR COLLISION RISK ASSESSMENT OF AN AIRSPACE FLOW CORRIDOR CONCEPT

Yimin Zhang, Ph.D.

George Mason University, 2014

Dissertation Director: Dr. John Shortle
\end{abstract}

This dissertation presents a methodology to estimate the collision risk associated with a future air-transportation concept called the flow corridor. The flow corridor is a Next Generation Air Transportation System (NextGen) concept to reduce congestion and increase throughput in en-route airspace. The flow corridor has the potential to increase throughput by reducing the controller workload required to manage aircraft outside the corridor and by reducing separation of aircraft within corridor. The analysis in this dissertation is a starting point for the safety analysis required by the Federal Aviation Administration (FAA) to eventually approve and implement the corridor concept.

This dissertation develops a hybrid risk analysis methodology that combines Monte Carlo simulation with dynamic event tree analysis. The analysis captures the unique characteristics of the flow corridor concept, including self-separation within the corridor, lane change maneuvers, speed adjustments, and the automated separation 
assurance system. Monte Carlo simulation is used to model the movement of aircraft in the flow corridor and to identify precursor events that might lead to a collision. Since these precursor events are not rare, standard Monte Carlo simulation can be used to estimate these occurrence rates. Dynamic event trees are then used to model the subsequent series of events that may lead to collision. When two aircraft are on course for a near-mid-air collision (NMAC), the on-board automated separation assurance system provides a series of safety layers to prevent the impending NNAC or collision. Dynamic event trees are used to evaluate the potential failures of these layers in order to estimate the rare-event collision probabilities.

The results show that the throughput can be increased by reducing separation to 2 nautical miles while maintaining the current level of safety. A sensitivity analysis shows that the most critical parameters in the model related to the overall collision probability are the minimum separation, the probability that both flights fail to respond to traffic collision avoidance system, the probability that an NMAC results in a collision, the failure probability of the automatic dependent surveillance broadcast in receiver, and the conflict detection probability. 


\section{CHAPTER 1 INTRODUCTION}

The flow corridor concept is a proposed route structure in en-route airspace to increase en-route capacity [JPDO 2012]. The concept is motivated by the projected growth of en-route demand. According to Federal Aviation Administration (FAA) forecast, the number of traveling passengers will grow from 731 million in 2010 to one billion in 2023. En-route operations will increase 1.8 percent annually, reaching 58.2 million aircraft handled in 2033 [FAA 2012].

The increased en-route demand will result in significant congestion and flight delays. According to an FAA study [FAA 2007], a 20\% increase in en-route demand will result in a 500\% increase in en-route delays (Table 1). A key reason for this increase is the limiting factor of controller workload. Each sector has a maximum number of aircraft that can be handled at one time, called the Monitor Alert Parameter (MAP). With increased demand, many more sectors will be above their MAP values, resulting in significant queuing delays. The flow corridor concept has the potential to increase enroute capacity and reduce delays by reducing the controller workload required to manage aircraft outside the corridor. 
Table 1 En-route delays with 2X demand [FAA 2007]

\begin{tabular}{|c|c|c|c|c|}
\hline Scenario & $\begin{array}{c}\text { En Route } \\
\text { Delays } \\
\text { (mins) }\end{array}$ & $\begin{array}{c}\text { Average En } \\
\text { Route Delays } \\
\text { (mins/Flight) }\end{array}$ & $\begin{array}{c}\text { Number of } \\
\text { Sectors } \\
\text { Exceeding } \\
\text { MAP }\end{array}$ & $\begin{array}{c}\text { Total Minutes } \\
\text { Exceeding MAP }\end{array}$ \\
\hline Baseline (2006) & 3,395 & 0.06 & 111 & 2,130 \\
\hline $20 \%$ Increase & 19,963 & 0.31 & 223 & 6,798 \\
\hline
\end{tabular}

\subsection{Evolution of Route Structure}

Flow corridors are the next step in the progression of the en-route route structure.

Figure 1 shows the historical progression of route structures and their associated surveillance technologies. In the early stages of aviation, there were no specific air routes. Aircraft followed landmarks on the ground, which were typically only visible during the daytime. After the introduction of very high frequency (VHF) omni-directional radio range (VOR), aircraft could stay on course by receiving radio signals transmitted by ground radio beacons. Distance measuring equipment (DME) allowed aircraft to measure ground projected distance by timing the VHF radio signals. With these technologies, an aircraft could follow the transmitted radio signals from one VOR to another, without visual landmarks. This increased capacity by allowing flights during the night time or during low-visibility weather conditions. These routes are called victor routes. Instrument flight rules (IFR) were established to manage flights during these conditions.

In 1956, the Civil Aeronautics Authority (CAA) introduced air-route surveillance radar to aviation. Aircraft equipped with a radar transponder were able to be located more precisely than before, allowing for aircraft to fly faster and closer to each other. With the 
new technology, a new type of route was introduced, called a jet route. Jet routes are located on higher altitudes than victor routes, thus increasing the potential volume of the airspace.

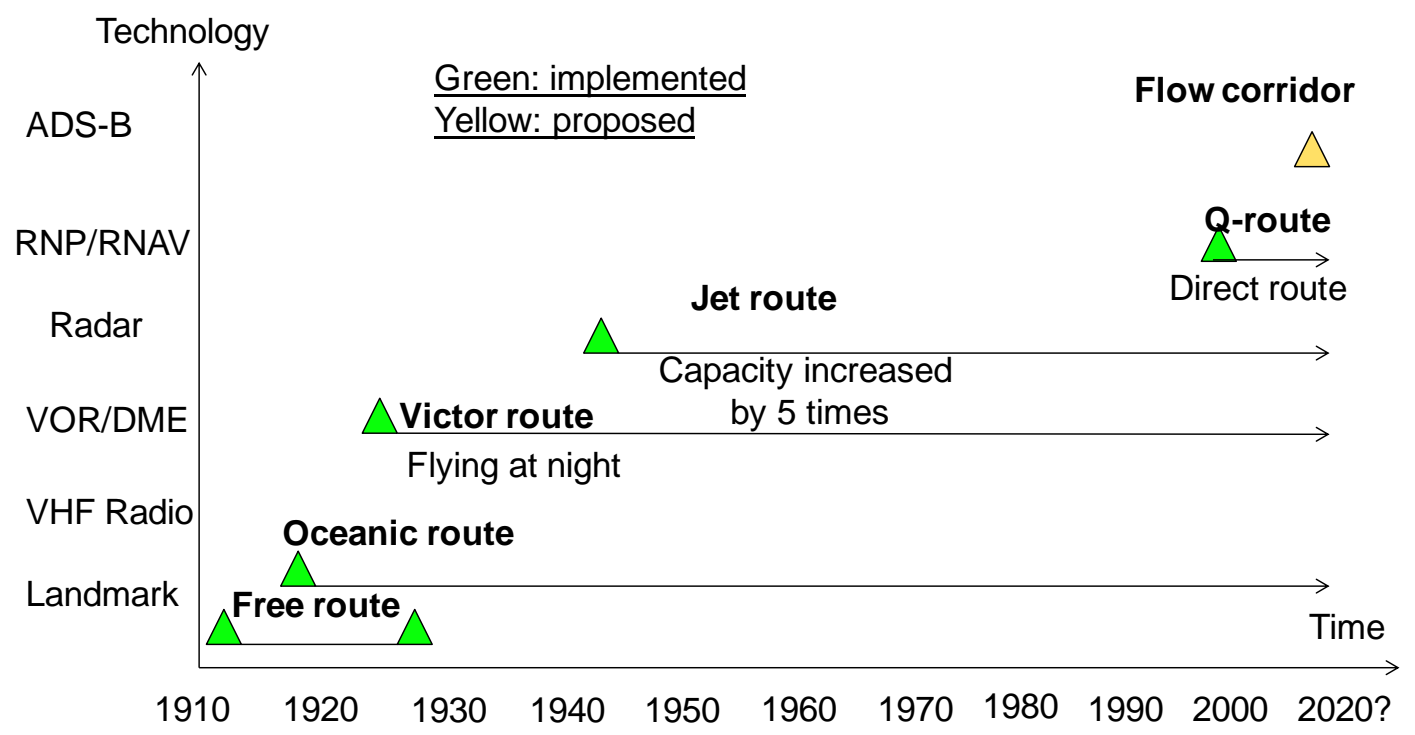

Figure 1 Evolution of route structure

The next step was the transition from jet routes to high altitude routes or Q-routes. Area navigation (RNAV) airborne equipment allows an aircraft to choose any course within a network of navigation beacons, rather than being restricted to navigating directly to and from the beacons. In 2000, a government-industry forum recommended increasing the use of area navigation (RNAV) to allow aircraft to more efficiently navigate within the National Airspace System (NAS). In 2003, the Code of Federal Regulations was changed to allow creation of RNAV routes in domestic airspace without being dependent on ground-based navigational aids (NAVAID). The first high altitude RNAV routes (Q routes) were established on the west coast later that year. 
Flow corridors are the next step in this progression, enabled by a new surveillance technology called automatic dependent surveillance-broadcast (ADS-B). With ADS-B, each aircraft receives its own state information (position and velocity) via a Global Navigation Satellite System such as GPS. Aircraft broadcast their own state information via ADS-B out and receive the state information of nearby aircraft via ADS-B in. Because each aircraft knows the state information of its surrounding traffic, conflict detection and resolution can occur on-board the aircraft without a central controller.

The process of conflict detection and resolution is completed by an automated separation assurance system currently under development. The system will need to detect conflicts and displays resolutions to the pilot. The pilot is then responsible to select and execute one of the candidate resolutions. Shifting separation assurance responsibility from the controllers to the pilots and the automation reduces the number of aircraft that must be separated by controllers during peak hours [Wing et al. 2008]. Capacity can also be increased by reducing in-trail separation between aircraft in the corridor via self separation capability and the onboard automated separation assurance system.

\subsection{Flow Corridor Concept-of-Operations}

A flow corridor is a long tube that contains en-route traffic flow. Figure 2 shows an example of a proposed flow corridor from Newark to Los Angeles [Wing et al. 2008]. Within the tube, the flow corridor consists of multiple closely-spaced parallel lanes. Figure 3 shows an example of 2 by 2 corridor with lanes separated in the lateral and vertical dimensions. (In this dissertation, only lateral configurations will be analyzed.) All fights in the corridor fly in the same direction. The path of the flow corridor can be 
adjusted based on weather information to follow the wind optimal routing. The flow corridor traffic is procedurally separated from other traffic. Non-flow corridor traffic cannot penetrate the corridor. Flights enter and exit the corridor via on-ramps and offramps. An on-ramp is a transition area that provides an extra parallel lane that merges into the corridor traffic flow.

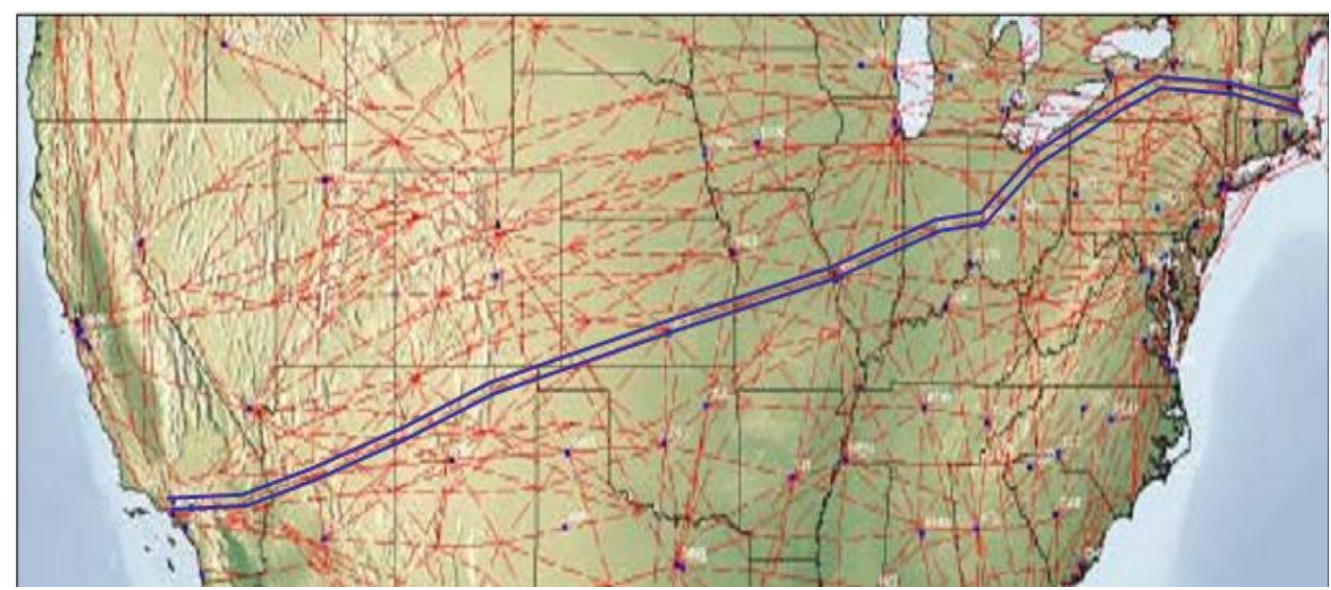

Figure 2 Example flow corridor [Wing et al. 2008]

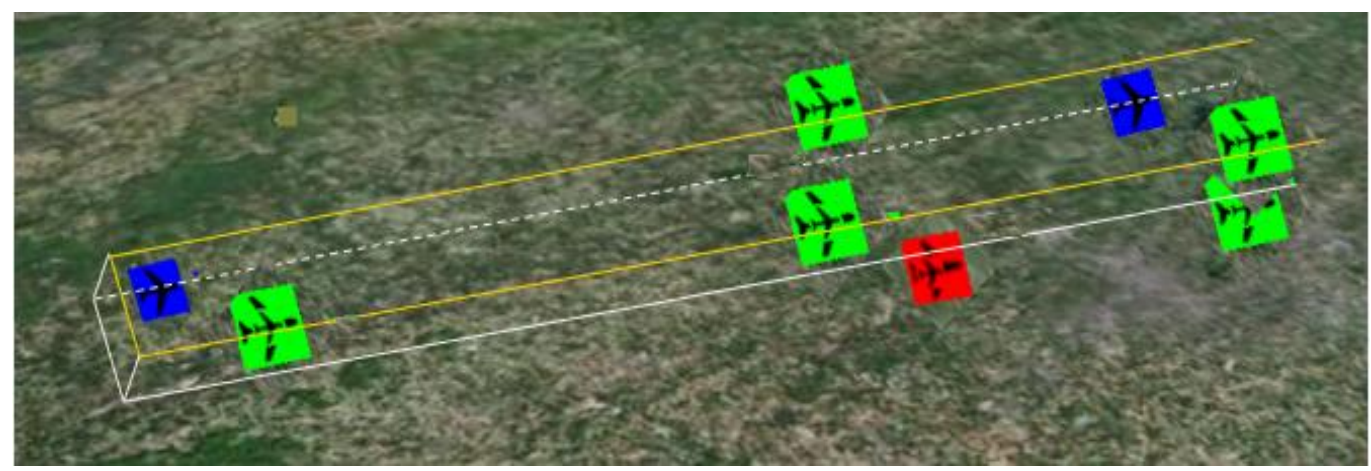

Figure 3 A 4-lane corridor 
To fly in the corridor, an aircraft must be equipped with required navigation performance (RNP), a self-separation capability and an onboard automated separation assurance system. RNP is a capability to fly along a specified path with a given level of lateral precision. Specifically, RNP- $x$ capability implies that an aircraft can fly within $\pm x$ nautical miles $(\mathrm{nm})$ of a centerline $95 \%$ of the time and within $\pm 2 x \mathrm{~nm}$ of a centerline $99.999 \%$ of time. For example, to fly within a 4-nm wide corridor $99.999 \%$ of the time (that is, within $\pm 2 \mathrm{~nm}$ of the centerline in either direction), an aircraft needs to be equipped with RNP-1. A 4-nm wide lane separation is highly reduced in the corridor compared to that of 8 nautical miles or more in current operations [Belle and Yousefi 2010].

Self separation allows aircraft to perform speed adjustments and lane change maneuvers without intervention by a central controller. Speed adjustments allow an aircraft to maintain separation with a leading aircraft in the same lane. The lane change capability allows a faster aircraft to switch lanes in order to pass a slower aircraft. The self-separation function is implemented by means of a locatability function in which ADS-B technology provides position and speed information of nearby flights.

Each flight is also equipped with an onboard automated separation assurance system. The onboard automated separation assurance system is assumed to follow the Autonomous Flight Management (AFM) concept that incorporates four levels of protection against a collision [Wing and Cotton 2011]. The first level, called the strategic intent-based conflict detection and resolution (CD\&R) function (SICDR), is designed to resolve conflicts 3 to10 minutes prior to a near mid-air collision (NMAC). Resolutions at 
this level take the flight plan into account and provide waypoints back to the original track. The second level, called the tactical intent-based CD\&R function (TICDR), is designed to resolve conflicts 2 to 3 minutes prior to NMAC. The tactical intent-based conflict resolution takes advantage of available intent data to provide longer look-ahead horizons compared to the tactical state-based conflict resolution (see below). It does not provide waypoints back to the original track. The TICDR function handles the conflicts that are not detected or resolved by the first level such as pop-up conflicts arising with little time left to solve. The third level, called the tactical state-based CD\&R function (TSCDR), is designed to resolve conflicts 1 to 2 minutes prior to NMAC. The TSCDR function only uses state-vector data for detecting conflicts. The conflict resolutions do not consider flight plans since the primary goal is to prevent collisions under a short time horizon. The last line of defense against a collision is the Traffic Collision Avoidance System (TCAS). TCAS is designed to resolve conflicts within 1 minute prior to the NMAC. Both flights should respond to TCAS resolutions to prevent the collision. TCAS is mandated by the International Civil Aviation Organization to be fitted to all aircraft with a maximum take-off mass of over $12,600 \mathrm{lbs}$.

The onboard automated separation assurance system is responsible to detect conflicts, generate conflict resolutions and display the resolutions to the pilots. It also alerts the pilots using an onboard speaker. The pilots are responsible to maintain safe separation with other aircraft. This includes maintaining situational awareness of nearby aircraft, following standard lane change procedures, and responding to conflict resolutions issued by the onboard automated separation assurance system. 
Figure 4 shows the relation between self separation and the automated separation assurance system. In this figure, aircraft 1 is assumed to be flying 20 knots faster than aircraft 2. Prior to any conflict alerts from the automated separation assurance system, the pilots perform self separation by monitoring the onboard display system. The onboard display system shows the speed and position information of nearby aircraft. At 20 minutes prior to an NMAC, the strategic intent-based function detects the conflict. In this example, 20 minutes corresponds to $6.7 \mathrm{~nm}$ separation. The potential conflict resolutions are to reduce speed or to change lanes to avoid the conflict given the position and speed information of nearby flights. The pilots must choose and execute a conflict resolution to avoid loss of separation.

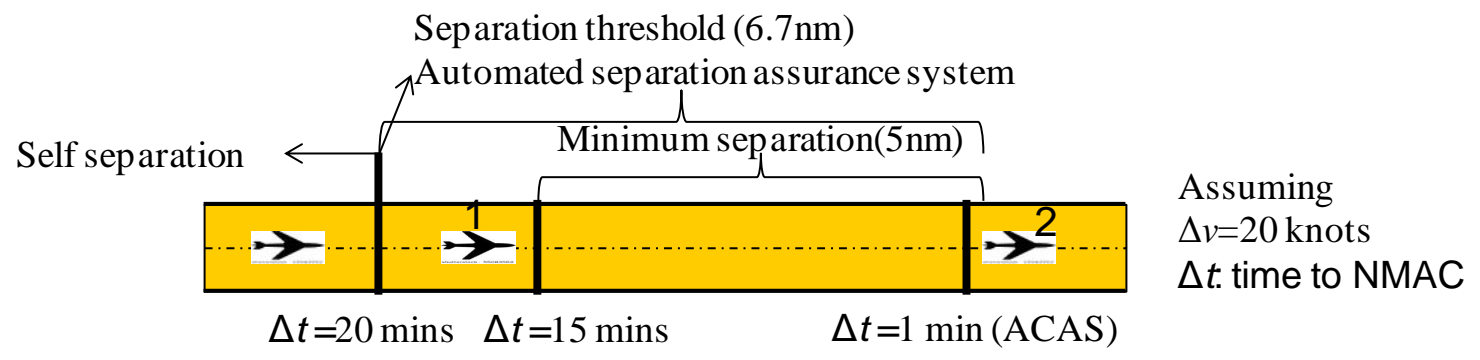

Figure 4 Relation between self separation and automated separation assurance system

\subsection{Problem Statement}

The main objective of this dissertation is to determine if the flow corridor concept-of-operations can meet a target level of safety. A quantitative methodology is needed to estimate the collision probability of the flow corridor concept-of-operations. If the flow corridor concept-of-operations satisfies the target level of safety using current 
separation standards (minimum separation $=5 \mathrm{~nm})$, then a second objective is to determine the minimum separation that satisfies the target level of safety. Key safety vulnerabilities of the flow corridor concept-of-operations are also sought.

Previous studies on the flow corridor concept-of-operations can be summarized into several categories:

1) Concept description, which presents the idea of flow corridors and how they operate [Alipio et al. 2003, Yousefi et al. 2004],

2) Geographical location, which identifies the potential routes for flow corridors [Sridhar et al. 2006, Kopardekar et al. 2007, Hoffman, et al. 2008, Xue and Kopardekar 2009],

3) Aircraft equipage, which discusses the required equipment and capabilities of aircraft in the corridors [Mundra and Simmons 2007, Wing et al. 2008, Yousefi et al. 2009],

4) Regulations, which discusses the rules and procedures that aircraft should follow when flying in the corridor [Mundra and Simmons 2007, Yousefi et al. 2009],

5) Benefit analysis, which shows the capacity gains that can be achieved using corridors [Wing et al. 2008, Yousefi et al. 2010].

However, none of these studies addresses the safety of the proposed corridor concept. References [Zhang et al. 2013, Ye et al. 2014] estimate the collision risk of the flow corridor concept. Zhang et al. [2013] use dynamic event tree to model the automated separation assurance system and consider different collision scenarios in the flow 
corridor. Ye et al. [2014] use a simulation to model aircraft lane changes and self separation in the flow corridor. The main gap is lack of an automated separation assurance system.

Other related safety studies include parallel tracks across the North Atlantic, have a similar parallel route structure. Because of the lack of radar coverage over the ocean, gross navigation errors along the North Atlantic tracks can be more than 25 nautical miles [NAT SPG 2013]. Such deviations may lead to collisions between aircraft on parallel tracks. The Reich collision risk model is widely used to estimate collision probabilities for such parallel tracks [Campos 2001; EUR 2012]. However, the Reich collision model does not account for many aspects of the flow corridor, such as lane change maneuvers, pilot control loops and failure of the onboard automated separation assurance system.

References [Shortle et al. 2012, Yousefi et al. 2012] conduct a safety analysis on the Automated Airspace Concept (AAC) and the AFM concept using dynamic event trees (DET). This dissertation considers the AFM concept as part of flow corridor concept. The difference is that this dissertation considers different types of conflict geometries when conducting safety analysis. Reference [Shortle and Zhang 2013] compares the safety of centralized and distributed automated separation concepts.

\subsection{Unique Contributions}

The dissertation provides an initial safety analysis of the flow corridor concept-ofoperations. This analysis can be used as a starting point for the required regulatory approval process. There are four major contributions. 
1. This research develops a methodology to estimate the collision probability of the flow corridor concept-of-operations. The methodology combines Monte Carlo simulation and dynamic event trees. The methodology considers self separation, lane change maneuvers, and the automated separation assurance system.

2. This research demonstrates that the flow corridor concept-of-operations can meet a target level of safety (5E-9 collisions per flight hour).

3. This research determines the trade-off between corridor capacity and the associated collision probability via reduction of in-trail separation.

4. This research determines key safety vulnerabilities.

\subsection{Summary of Results}

This dissertation estimates the collision rate associated with a flow corridor that has two parallel lanes at the same altitude. The collision rate is decomposed into three parts: the occurrence rate of potential NMAC trajectories, the probability of an actual NMAC trajectory, and the conditional probability of a collision given an actual NMAC trajectory. An actual NMAC trajectory is an event in which two aircraft are on course for an NMAC. A potential NMAC trajectory occurs at the decision point where an erroneous decision would result in an actual NMAC trajectory.

Monte Carlo simulation shows that the occurrence rate of potential NMAC trajectories is affected by minimum separation, fleet mix, initial separation, standard deviation of target velocity, and relative velocity threshold. A sensitivity analysis shows that the minimum-separation parameter has the largest impact on the overall occurrence rate of potential NMAC trajectories in an overtaking collision scenario (when a faster 
aircraft collides with a leading slower aircraft). The fleet-mix parameter has the largest impact on the occurrence rate of NMAC trajectories in a lane-change collision scenario (due to a higher frequency of lane changes with a greater mixture of traffic).

The dynamic event trees calculate the conditional collision probability given that two aircraft are on course for an NMAC. A sensitivity analysis identifies critical factors that have a high impact on the collision probability. These are the probability that both flights fail to respond to TCAS, the failure probability of the ADS-B in receiver, and the conflict detection probability. The trade-off curve between collision probability and throughput suggests that the throughput can be safely increased by reducing separation to 2 nautical miles. The overall collision probability is $8.55 \mathrm{E}-10$ per flight hour using current separation standards (minimum separation $=5 \mathrm{~nm}$ ). The collision-capability tradeoff shows that the collision probability increases by $5 \mathrm{E}-10$ when the throughput increases by 30 aircraft per hour. 


\section{CHAPTER 2 LITERATURE REVIEW}

This chapter discusses literature related to analyzing the safety of the flow corridor concept-of-operations. Section 2.1 describes research specifically related to the flow corridor concept. Section 2.2 discusses collision risk models for parallel route structures. Section 2.3 discusses safety analyses of automated separation assurance systems. Section 2.4 discusses safety analyses of the traffic collision avoidance system. Section 2.5 discusses general safety analysis techniques.

\subsection{Flow Corridor Concept}

Table 2 summarizes research related to the flow corridor concept. The main areas of research are: Concept description, geographical location, aircraft equipage, regulation and attributes design, benefit analysis, and safety analysis. Each area is discussed in more detail in later sub-sections. The main gap in this literature is that little has been done to analyze the safety and collision risk associated with the flow corridor concept. 
Table 2 Flow corridor research areas

\begin{tabular}{|c|c|c|c|c|c|c|}
\hline $\begin{array}{c}\text { Paper/ } \\
\text { Category }\end{array}$ & $\begin{array}{c}\text { Concept } \\
\text { description }\end{array}$ & $\begin{array}{c}\text { Geo- } \\
\text { graphical } \\
\text { location }\end{array}$ & $\begin{array}{c}\text { Aircraft } \\
\text { capacities } \\
\text { and } \\
\text { equipage }\end{array}$ & $\begin{array}{l}\text { Regulation } \\
\text { and } \\
\text { attributes } \\
\text { design }\end{array}$ & $\begin{array}{l}\text { Benefit } \\
\text { analysis }\end{array}$ & $\begin{array}{l}\text { Corridor } \\
\text { safety } \\
\text { analysis }\end{array}$ \\
\hline $\begin{array}{c}\text { Alipio, J. } \\
2003\end{array}$ & $\mathrm{x}$ & & $\mathrm{x}$ & & & \\
\hline $\begin{array}{l}\text { Yousefi, A. } \\
2004\end{array}$ & $\mathrm{X}$ & $\mathrm{X}$ & & & & \\
\hline $\begin{array}{l}\text { Sridhar, B. } \\
2006\end{array}$ & $\mathrm{X}$ & $\mathrm{X}$ & & & & \\
\hline $\begin{array}{l}\text { Kopardekar } \\
\text {, P. } 2007\end{array}$ & $\mathrm{x}$ & & $\mathrm{x}$ & & & \\
\hline $\begin{array}{c}\text { Mundra, A. } \\
2007\end{array}$ & $\mathrm{x}$ & & $\mathrm{x}$ & $\mathrm{X}$ & & \\
\hline $\begin{array}{l}\text { Hoffman, } \\
\text { R. } 2008\end{array}$ & $\mathrm{X}$ & $\mathrm{X}$ & $\mathrm{X}$ & $\mathrm{X}$ & & \\
\hline $\begin{array}{l}\text { Xue, M. } \\
2008\end{array}$ & & $\mathrm{X}$ & & & & \\
\hline $\begin{array}{c}\text { Wing, D. } \\
2008\end{array}$ & $\mathrm{x}$ & $\mathrm{x}$ & $\mathrm{x}$ & $\mathrm{X}$ & $\mathrm{x}$ & \\
\hline $\begin{array}{l}\text { Yousefi, A. } \\
2009\end{array}$ & & & $\mathrm{x}$ & & $\mathrm{x}$ & \\
\hline $\begin{array}{l}\text { Yousefi, A. } \\
2010\end{array}$ & & & & $\mathrm{x}$ & & \\
\hline $\begin{array}{l}\text { Ye, B. } \\
2013\end{array}$ & & & & & $\mathrm{X}$ & $\mathrm{x}$ \\
\hline
\end{tabular}

\subsubsection{Geographical Location}

This body of research tries to identify where flow corridors should be located to accommodate the most traffic within high density airspace.

Yousefi et al. [2004] analyze operations between city pairs in the NAS. A network of High-volume Tube-shaped Sectors (HTS) is proposed. The objective is to reduce sector congestion and increase throughput. Potential locations of the HTS are between busy origin and destination (OD) city pairs with more than 60 operations per day. 
Given a candidate HTS, the optimal routing of the HTS is determined by user-preferred vectoring. Close proximity routes can be combined into a single traffic flow - for example, LAX to BOS and LAX to ORD. The traffic flow between OD pairs is modeled as a fluid. Each flight in the flow is part of the fluid with velocity vectors. The optimal vector along the flow is the optimal routing of the HTS.

Xue and Kopardekar [2008] use a Hough transform to identify the potential routing of flow corridors. This method is widely used in image processing to find lines in a given image. Here, the method is used to find groups of flight tracks that have the highest density in the National Airspace System (NAS). One day of track data $(62,143$ flights) are extracted from FAA's Aircraft Situation Display to Industry (ASDI). The data are transformed into points in Hough space, where each point represents one great circle flight route. Points with close proximity are clustered into grids. The grids with the highest density are the candidates of flow corridors. 60 corridor candidates are identified.

Sridhar et al. [2006] consider tubes that connect the 18 busiest regions as the flow corridor candidates. Each region includes a group of airports. Hoffman et al. [2008] construct a tube network based on user preferred routes and ATC preferred routes. Wing et al [2008] consider a single coast-to-coast routing, linking Newark (EWR) airport with San Francisco (SFO) airport.

\subsubsection{Aircraft Capabilities and Equipage}

This body of research specifies the required equipage and capabilities for aircraft in the corridor. The aircraft shall be equipped with ADS-B to broadcast and receive state information (speed and position); the aircraft in the flow corridor shall be capable of RNP 
and equipped with automated separation assurance system [Alipio et al. 2003, Kopardekar et al. 2007, Hoffman et al. 2008, Wing et al. 2008, Yousefi et al. 2009]. All references specify the same requirements except that Mundra and Simmons [2007] suggest that the flow corridor concept shall be capable of self separation. Self separation allows aircraft to perform speed adjustments and lane change maneuvers without intervention by a central controller. Speed adjustments allow an aircraft to maintain separation with a leading aircraft in the same lane. The lane change capability allows a faster aircraft to switch lanes in order pass a slower aircraft. RNP allows aircraft to fly within the lane $99.999 \%$ of time. The onboard automated separation assurance system is responsible to detect conflicts, generate conflict resolutions and display resolutions to

pilots. Both the self-separation capability and the automated separation assurance system require the locatability function that provides position and speed information of nearby flights. ADS-B in and out can provide such information which is displayed on Cockpit Display Traffic Information (CDTI).

\subsubsection{Design Attributes and Procedures}

This body of research identifies the design attributes of the flow corridor, their alternatives, and associated procedures.

Table 3 shows a set of design attributes for a corridor concept called the dynamic multi-track airway (DMA) [Wing et al. 2008]. For example, two types of track configuration are identified. One is a speed-based configuration in which each track is designated with a nominal Mach number. The other is a speed-independent with passing configuration in which faster aircraft can change lanes in order to pass slower aircraft. 
Simulation results in this study show that the speed independent configuration performs better at lower track loadings, but worse at higher track loadings due to aircraft flying non-optimal speeds.

Table 3 Corridor design attributes and alternatives [Wing et al. 2008]

\begin{tabular}{|c|c|}
\hline Corridor design attributes & Corridor design alternatives \\
\hline Location of entry and exit & $\begin{array}{l}\text { (a) Close to major airports } \\
\text { (b) User preferred location }\end{array}$ \\
\hline Track configuration & $\begin{array}{c}\text { (a) Speed-based } \\
\text { (b) Speed-independent with passing }\end{array}$ \\
\hline Altitude stratification & $\begin{array}{l}\text { (a) Less than six } \\
\text { (b) All upper flight level }\end{array}$ \\
\hline Aircraft capability & $\begin{array}{c}\text { (a) Required navigation performance } \\
\text { (RNP-x) } \\
\text { (b) Automated separation assurance } \\
\text { system (AAC, AFM) }\end{array}$ \\
\hline Separation responsibility & $\begin{array}{l}\text { (a) Sector controller } \\
\text { (b) Corridor controller } \\
\text { (c) Pilots }\end{array}$ \\
\hline $\begin{array}{l}\text { Rerouting for convective weather and } \\
\text { congestion }\end{array}$ & $\begin{array}{l}\text { (a) Uplink rerouted trajectories to all } \\
\text { aircraft } \\
\text { (b) Follow the leader }\end{array}$ \\
\hline
\end{tabular}

The lane change maneuver is a unique procedure in the flow corridor concept-ofoperations. To prevent an operational error during the maneuver, Yousefi et al. [2010] discuss a nominal procedure design for the lane change maneuver. The nominal procedure to change lanes is that: a) The aircraft broadcasts to all other aircraft its intent to change lanes and maintains a constant flight profile during the procedure, b) the aircraft makes a 30 degree course to intercept the target lane, c) when the aircraft reaches the centerline of the target lane, it broadcasts to all other aircraft that it has completed the procedure. When an aircraft performs the lane change maneuver, nearby aircraft should 
maintain their flight status (such as speed) to avoid conflicts. Mundra and Simmons [2007] discuss the procedure of entering a flow corridor. Hoffman et al. [2008] discuss a list of design attributes and their alternatives including on-ramps/off-ramps, separation responsibilities, etc.

\subsubsection{Benefit and Cost studies}

This body of research tries to identify the potential benefits that can be achieved using corridors. Studies have shown that sector loads and delays can be reduced under future traffic conditions by implementing flow corridors connecting major regions [Wing et al. 2008]. Figure 5 shows the reduction of traffic loads of the top 50 en route sectors under a $2 \mathrm{X}$ demand scenario with one implemented flow corridor. The baseline demand is based on a moderate-demand, good-weather day including 57,093 flights. Most of the sector loads are reduced below their sector MAP values. It is expected that further reductions can be achieved by implementing multiple flow corridors. 


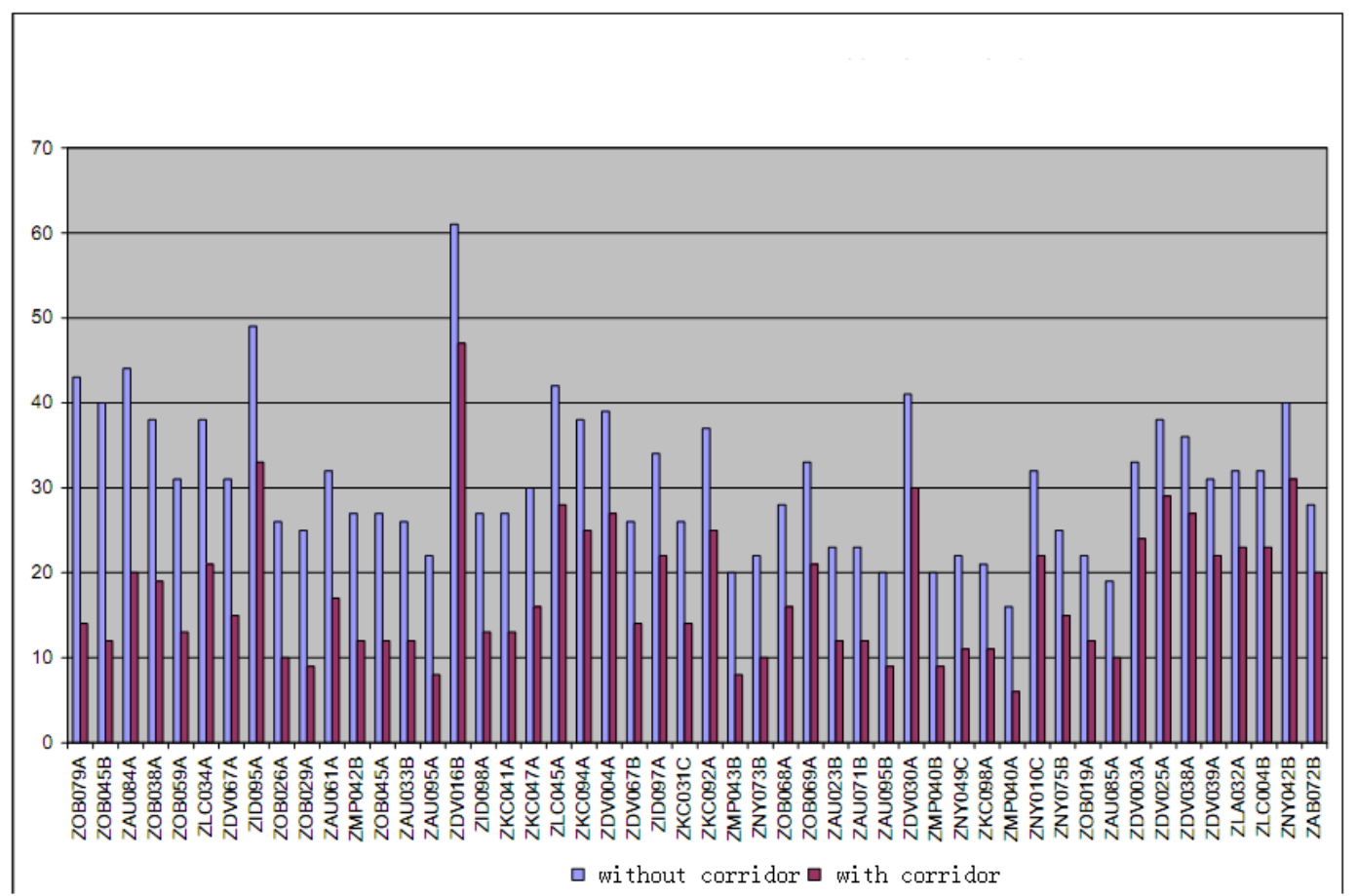

Figure 5 Flights comparison during peak hour for 2X demand [Wing et al. 2008]

In Yousefi et al. [2010], en-route delays under current operations are calculated using the NAS Simulation and Queuing Model. This model uses predetermined sector capacity values and applies airport holding, airborne delay and sector demands. The flow corridor is implemented as a user-preferred route. It assumes that the flow corridor has enough capacity to allow aircraft to fly their optimal speeds. The flow-corridor delays are the extra distance to join and leave flow corridor. Figure 6 shows the relation between the number of corridors and the percentage of delay reduction. NAS wide delays are reduced by $60 \%$ if 10 coast-to-coast corridors are implemented. 


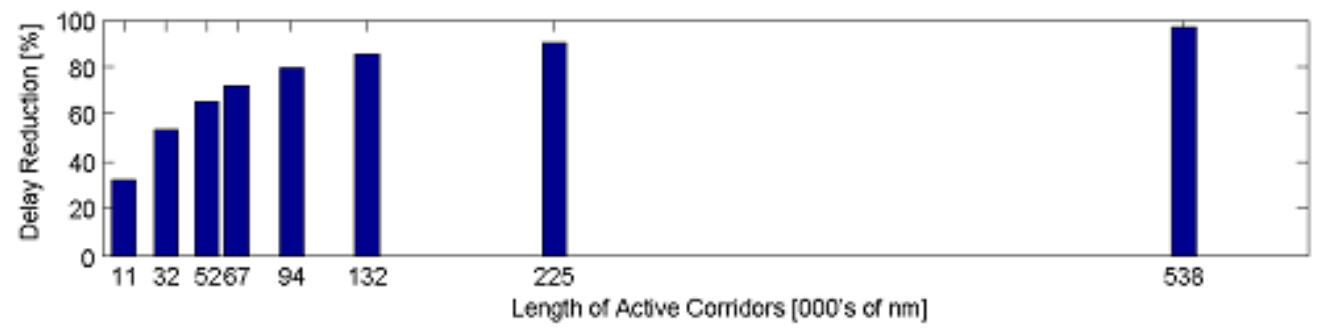

Figure 6 NAS wide delay reduction with length of flow corridors [Yousefi et al. 2010]

\subsubsection{Summary}

While the corridor concept has been studied with respect to a number of topics,

little has been done to address the safety of the corridor. Ye et al. [2013] discuss the trade-off between conflict risk and capacity for a 2-lane flow corridor. This study includes the self separation capability and lane change maneuvers. However, it does not include the automated separation assurance system and does not directly estimate collision probabilities.

\subsection{Collision Risk Models for Parallel Routes}

This section discusses collision risk models for parallel routes. Figure 7 shows that collision risk models have been used on parallel routes, automated separation assurance concepts, and TCAS. Parallel routes have a similar structure as flow corridors. The flow corridor concept requires equipage of the automated separation assurance system and TCAS. 


\begin{tabular}{|c|c|c|c|c|}
\hline & & Paper & $\begin{array}{c}\text { Collision risk } \\
\text { models }\end{array}$ & Limitations \\
\hline \multirow{5}{*}{\multicolumn{2}{|c|}{ Parallel routes }} & Carreno, 2007 & \multirow{5}{*}{ Reich model } & \multirow{5}{*}{$\begin{array}{c}\text { 1. No good for collision } \\
\text { assessement in longitutude direction } \\
\text { 2. No automated separation } \\
\text { assurance system } 3 \text {. No human } \\
\text { behavious }\end{array}$} \\
\hline & & NYVI, 2010 & & \\
\hline & & Moek, 2010 & & \\
\hline & & Blom, 1999 & & \\
\hline & & Everdij, 2006 & & \\
\hline \multirow{5}{*}{$\begin{array}{c}\text { Automated } \\
\text { separation } \\
\text { assurance } \\
\text { concept }\end{array}$} & \multirow{2}{*}{$\mathrm{AFM}$} & Blom, 2006 & \multirow{2}{*}{$\begin{array}{l}\text { TOPAZ, } \\
\text { SafeATAC }\end{array}$} & \multirow{5}{*}{$\begin{array}{c}\text { 1. No lane change maneuvers } 2 \text {. No } \\
\text { conflict geometries with respect to } \\
\text { flow corridor concept }\end{array}$} \\
\hline & & Yousefi, 2012 & & \\
\hline & \multirow{3}{*}{$\mathrm{AAC}$} & Blum, 2010 & \multirow{3}{*}{$\begin{array}{l}\text { Monte Carlo } \\
\text { simulation, } \\
\text { Markov chain, } \\
\text { dynamic event } \\
\text { tree }\end{array}$} & \\
\hline & & Volovoi, 2011 & & \\
\hline & & Shortle, 2012 & & \\
\hline \multirow{2}{*}{\multicolumn{2}{|c|}{ TCAS }} & MITRE, 1984 & \multirow{2}{*}{$\begin{array}{l}\text { Simulation, fault } \\
\text { tree }\end{array}$} & \multirow{2}{*}{$\begin{array}{l}\text { No other features of flow corridor } \\
\text { concept }\end{array}$} \\
\hline & & Kuchar, 2004 & & \\
\hline
\end{tabular}

Figure 7 Collision risk models

\subsubsection{Parallel Routes}

Parallel routes are similar to the flow corridor in terms of the route structure. One example is the set of North Atlantic tracks. These tracks connect the northeast of North America with Western Europe across the Atlantic Ocean. Because of the lack of radar coverage, lateral position and velocity errors are significantly increased. The gross navigation errors can be more than 25 nautical miles. Thus, a basic research question for parallel routes is to determine the safe lateral and vertical separation. Figure 8 shows a 2lane parallel route. A collision might occur when the aircraft on the left lane deviates to the right and the aircraft on the right lane deviates to the left. Analyses have been done to determine the safe distance between the two lanes based on the probability distributions of these deviations. A widely used model is the Reich collision risk model. 


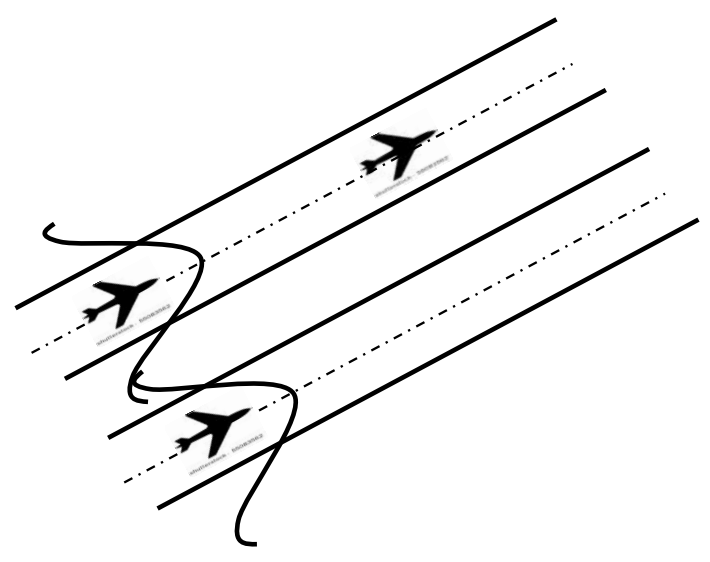

Figure 8 2-lane parallel route

\subsubsection{Reich Model}

The Reich Collision Risk Model, developed by Reich [1966] was originally developed to estimate collision risk for oceanic traffic over the North Atlantic and to determine the appropriate lateral separation between aircraft [Campos 2001, Campos and Marques 2002]. It also has been used to evaluate collision risk and perform numerous safety assessments approved by ICAO. For example, it has been applied to determine appropriate vertical separation for North Atlantic tracks. It has also been used to estimate collision probability for four EUR-SAM corridors in the South Atlantic [EUR 2012]. The fundamental input is the probability distribution of the deviations.

The main assumptions of the Reich model are: 1) The lateral/vertical deviations of aircraft on adjacent tracks are uncorrelated. 2) The lateral/vertical speed of an aircraft is not correlated with its lateral/vertical deviation. 3) The protected zone of an aircraft is a 
rectangular box. 4) There is no corrective action by pilots to avoid the collision. Then collision rate between aircraft on adjacent tracks is given by the following equation.

Equation 1 Expected lateral collisions per flight hour

$N_{\text {ay }}=P_{y}\left(S_{y}\right) P_{z}(0) \frac{\lambda_{x}}{S_{x}}\left\{\mathrm{E}_{\text {ysame }}\left[\frac{|\Delta \bar{v}|}{2 \lambda_{x}}+\frac{|\overline{\dot{y}}|}{2 \lambda_{y}}+\frac{|\overline{\dot{z}}|}{2 \lambda_{z}}\right]+\mathrm{E}_{\text {yopposite }}\left[\frac{2|\bar{v}|}{2 \lambda_{x}}+\frac{|\overline{\dot{y}}|}{2 \lambda_{y}}+\frac{|\overline{\mathrm{z}}|}{2 \lambda_{z}}\right]\right\}$

- $N_{a y}$ is the expected number of accidents per flight hour due to the loss of lateral separation between aircraft flying on tracks with nominal spacing $\mathrm{S}_{y}$.

- $\quad S_{\mathrm{y}}$ is the lateral separation of the track centerlines.

- $P_{y}\left(S_{y}\right)$ is the probability of lateral overlap of aircraft nominally flying on the lateral adjacent paths at the same flight level.

- $P_{z}(0)$ is the probability of vertical overlap of aircraft nominally flying at the same flight level.

- $\mathrm{E}_{\mathrm{ysame}}$ is the same direction lateral occupancy, i.e. the average number of same direction aircraft flying on the laterally adjacent tracks at the same flight level within segments of length $2 S_{\mathrm{x}}$, centered on the typical aircraft.

- $\mathrm{E}_{\mathrm{yopposite}}$ is the opposite direction lateral occupancy, i.e. the average number of opposite direction aircraft flying on laterally adjacent tracks at the same flight level within segments of length $2 S_{\mathrm{x}}$, centered on the typical aircraft.

- $S_{x}$ is the length of the longitudinal window used in the calculation of occupancies.

- $\lambda_{x}$ is the average length of an aircraft. 
- $\lambda_{y}$ is the average width of an aircraft

- $\lambda_{z}$ is the average height of an aircraft

- $|\Delta \bar{v}|$ is the average relative along-track speed of two aircraft flying at the same flight level in the same direction.

- $|\bar{v}|$ is the average ground speed of an aircraft.

- $|\bar{y}|$ is the average lateral cross-track speed between aircraft that have lost their lateral separation.

- $|\bar{z}|$ is the average relative vertical speed of aircraft flying at the same flight level. The main limitation of the Reich model is that it does not account for lane change maneuvers, pilot control loops, or failures of the onboard automated separation assurance system.

Some examples of studies that use the Reich collision model are the following. Moek and Harrison [1992] use the same method on reduced Vertical Separation Minima of South Atlantic tracks. Campos [2001] discuss the collision probability between aircraft with dissimilar position errors using the Reich model. Campos and Marques [2011] compare the lateral collision probabilities given three kinds of distribution for position deviation using the Reich model.

\subsubsection{Reich Model Extensions}

Bakker and Blom [1993] derive a generalized Reich model by removing the assumption that all directions of position and speed deviation are mutually independent. Daams et al. [1999] use the generalized Reich collision model with hybrid state Markov processes and Petri Net models to model a 2 lane parallel route. The model considers 
aircraft performance of navigation, communication, and response times in the control loop. Due to the high complexity of the model, analytical evaluation of the accident risk is not feasible. Monte Carlo simulation is not feasible either in terms of the low probability of collisions. Therefore, the total collision risk is decomposed into different conditions. The Monte Carlo simulation provides the distribution of aircraft position and speed. Then the generalized Reich collision model is used to calculate the collision probability from these distributions.

Shortle et al. [2004] apply the generalized Reich model to terminal areas. The landing process to a non-towered airport is modeled using dynamically colored Petri nets. The simulation provides the distribution for the relative position and velocity of an aircraft pair. Then the collision probability is calculated using the generalized Reich collision model.

\subsubsection{Summary}

The Reich collision risk model is widely used to estimate collision probabilities on parallel tracks with large flight path deviations. The flow corridor concept also has a parallel route structure. However, the flow corridor has additional complexities which are not covered by the Reich model. First, the Reich model does not include lane change maneuvers because it does not account for human behaviors. It mainly emphasizes navigation performance. Second, the Reich model does not include the onboard conflict detection and resolution functions since it is difficult to model the equipment failures. 


\subsection{Automated Separation Assurance Systems}

One of the requirements of the flow corridor concept is an automated separation assurance system. Two automated separation assurance systems are widely studied, the Advanced Airspace Concept (AAC) [Erzberger 2001] and the Autonomous Flight Management concept (AFM) [Wing and Cotton 2011]. The main difference is that the $\mathrm{AAC}$ is a ground-based centralized system and the AFM is an airborne-based decentralized system. The AAC system has a central computer on the ground that detects conflicts and sends conflict resolutions to pilots via data link. The AFM system consists of primarily the onboard equipment. The AFM concept is assumed as part of the corridor concept in this study because the AFM concept does not include the air traffic controller in the control loop. The pilots take responsibility to maintain separation and prevent collisions. This meets the requirement of the flow corridor concept to reduce controller workload.

\subsubsection{Autonomous Flight Management Concept}

AFM is a distributed concept in which each aircraft is equipped with an onboard automation system to provide separation assurance maneuvers without a centralized controller. Associated with AFM is a new flight status called Autonomous Flight Rules (AFR). Flights under AFR have some important differences from Instrument Flight Rules (IFR) and Visual Flight Rules (VFR). For example, flights under AFR are responsible to maintain separation from all other aircraft in the airspace, including IFR, VFR and other AFR flights. In contrast, controllers are responsible for maintaining separation for IFR flights. Second, AFR aircraft may select flight trajectories that more closely match the business case optimum instead of following flight plans and controller instructions like 
IFR flights. AFR flights can also change flight status to IFR when entering a constrained airspace and hand over separation responsibilities to controllers.

The onboard conflict detection and resolution system [Wing et al. 2010] in the AFM concept collects information from different surveillance sources. For example, the ADS-B system on an aircraft provides the position information of the aircraft (ownship) and the other AFR flights around it. The Traffic Information Service - Broadcast (TIS-B) system collects position information of all flights, including non-AFR flights (thus, if TIS-B fails, the onboard CD\&R system is not able to locate non-ADS-B aircraft). The Flight Information Service-Broadcast (FIS-B) system provides meteorological and aeronautical information. The onboard CD\&R system also receives information such as required time of arrival and constrained airspace from Controller-Pilot Data Link Communications (CPDLC). Pilots give input to the onboard CD\&R system through the Mode Control Panel (MCP). With all these data, the onboard CD\&R system is able to detect conflicts that may occur in the near future and provide a conflict free trajectory resolution. An AFM concept diagram is shown in Figure 9 [Wing and Ballin 2004]. 


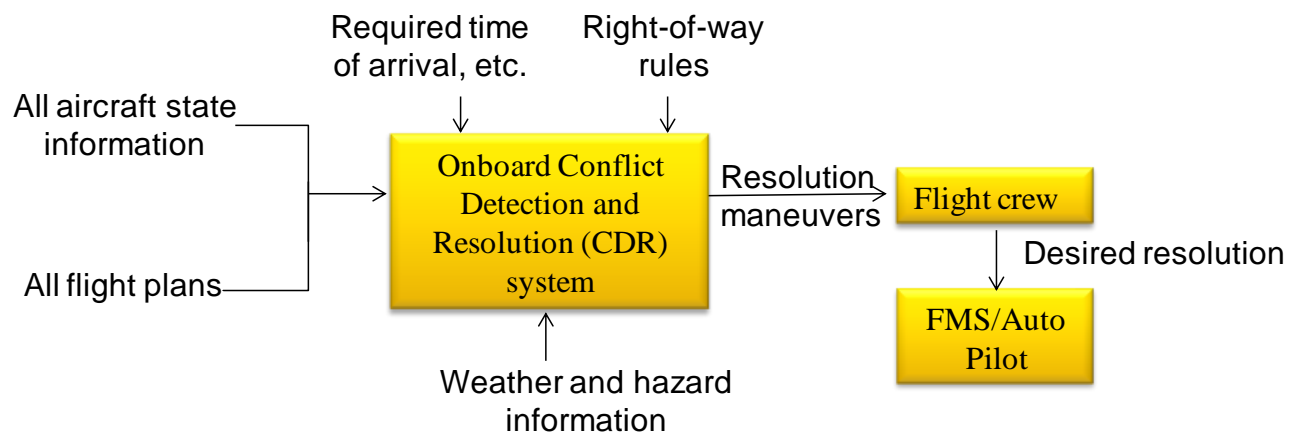

Figure 9 AFM concept diagram [Wing and Ballin 2004]

The onboard CD\&R system contains four functions that are responsible to detect and resolve conflicts in four different time frames (Figure 10). The $x$-axis denotes the time prior to a potential NMAC.

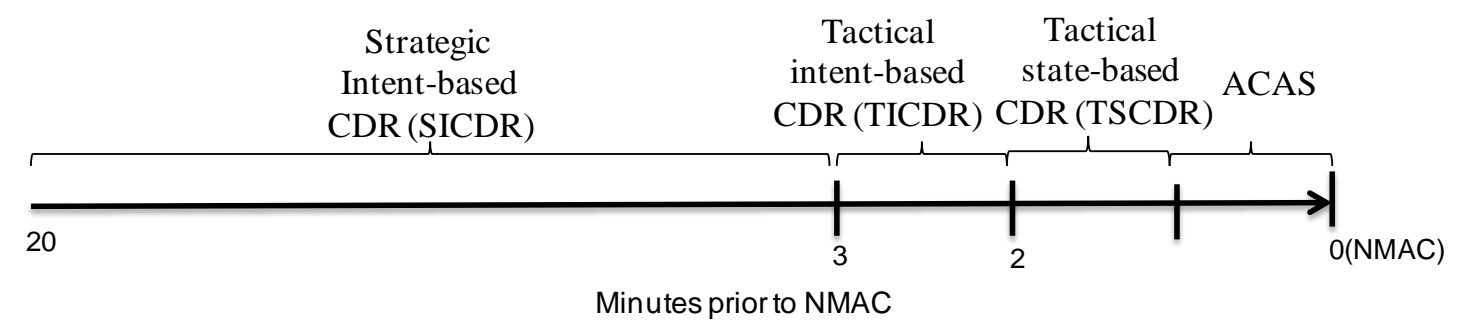

Figure 10 Onboard CD\&R system

Table 4 summarizes the main functions of the AFM concept and the associated systems and components. 
Table 4 AFM functions and components

\begin{tabular}{|c|c|}
\hline Functions & Components \\
\hline Main Surveillance & ADS-B \\
\hline Backup Surveillance & TIS-B \\
\hline Navigation & GPS \\
\hline Communication & FIS-B, CPDLC, voice \\
\hline Conflict Detection & Strategic CD, tactical CD \\
\hline Conflict Resolution & Strategic CR, tactical CR \\
\hline Display & Navigation display \\
\hline
\end{tabular}

\subsubsection{Safety Analysis of AFM Concept}

This dissertation considers the AFM concept as part of the flow corridor conceptof-operations. The literature on safety analysis of the AFM concept provides insights on the collision risk assessment of the flow corridor concept-of-operations. The methodologies used in the literature include Monte Carlo simulation, Petri-net diagrams, dynamic event trees, and reliability diagrams. The basic idea is to model the conflict detection and resolution capability in order to estimate the collision probability.

Blom et al. [2005] estimate the collision risk of the free flight concept using Monte Carlo simulation with Petri Nets (PN). The free flight airspace assumes that the pilots have the responsibility to avoid conflicts, assisted by the navigation system, surveillance processing and equipment displaying conflict-solving trajectories. Three conflict scenarios are analyzed: 1) Two aircraft with a head-on encounter at the same flight level. The results from the simulation show that the conflict detection and resolution function work effectively with an approximate NMAC probability of 1E-9. 2) Eight aircraft. The collision probability increases to $1 \mathrm{E}-6$. The key reason is that uncoordinated resolution maneuvers result in new conflicts. 3) The last scenario 
simulates equipped aircraft flying randomly. The collision probability is $1.6 \mathrm{E}-4$. The main limitation of this analysis is that it is time consuming to simulate a collision in terms of the low occurrence rates of failures.

Consiglio et al. [2007] discuss a simulation to test the safety performance of AFM-equipped aircraft flying in very high density airspace. Aircraft are assumed to fly at the same altitude. The simulation platform includes a simulation manager, a background traffic generator, and an aircraft model that includes a 6 degrees-of-freedom aircraft dynamics model, an FMS model, an ADS-B model, and an airborne separation automation model. The FMS model and ADS-B model are inputs to the airborne separation automation model, which provides flight intent, and speed and position information. Overall, the results show that airborne separation can be safety performed under the assumptions and environment of the simulation. As expected, the rate of conflicts increases with traffic density. The percentage of small angle conflicts is less than other angles. A potential reason could be the limited test airspace and simulation settings. A very low probability is found for downstream secondary conflicts (i.e., events where the resolution of one conflict leads to a new secondary conflict). The main limitations of this work relative to corridor safety are (a) the work does not model the specific geometries and dynamics of the corridor (lane changes, etc.) and (b) it does not consider failures of the CD\&R functions. Dynamic event trees, used in the next reference, can overcome the latter weakness.

Yousefi et al. [2012] use dynamic event trees to model the process of two enroute aircraft resolving a conflict, given trajectories that are on course for an NMAC. The 
time flow of the dynamic event tree is divided into four parts, representing different time frames of the AFM concept (in this paper, strategic conflict detection and resolution, tactical conflict detection and resolution, TCAS, and pilot see and avoid). The results show that the probability of an NMAC is about $1.1 \mathrm{E}-9$, given a sector density of 40 aircraft over a 15 minute time interval. A limitation of this study is that the dynamic event tree analysis only considers a pair of aircraft and not the full state information of all aircraft in a corridor. Thus, it does not model, for example, the relationship between fleet mix and lane changes. Also, the study does not specify the geometry of trajectories that are on course for an NMAC. For example, a head-on encounter may have a higher conflict detection probability, but less time prior to the NMAC. In the flow corridor, it is possible to enumerate all of the conflict geometries.

\subsubsection{Conflict Detection and Resolution Algorithms}

A key element of an automated separation assurance system is the system for detecting and resolving conflicts. This section discusses some of the relevant literature on conflict detection. The conflict detection and resolution algorithm must provide reliable alerts to the pilot. For example, if the automation provides too many false alerts, pilots may not trust the alert. In addition, the conflict alert should provide enough time for the pilot to execute a resolution.

This dissertation uses a widely studied probabilistic method for conflict detection, developed by Erzerger et al. [1997]. The method models the position error with a straightforward modeling of uncertainty. It is also validated by simulations and experimentation on real traffic. To estimate the conflict probability, the prediction error 
for the across-track dimension is treated as a normal random variable with a constant standard deviation between 0.5 and $1.0 \mathrm{~nm}$. Along-track prediction errors are assumed to increase linearly as a function of time, as shown in Equation 2 where $\mathrm{N}(0,1)$ is a standard normal distribution.

Equation 2 Along-track position predication $x(t)=v_{N} t+\operatorname{CtN}(0,1)$

Figure 11 shows the prediction error graphically for two aircraft flying perpendicular to each other. As the along track position uncertainties grow with time, the prediction error transforms from a circle to an ellipse. The goal is to calculate the conflict probability when two ellipses cross each other.

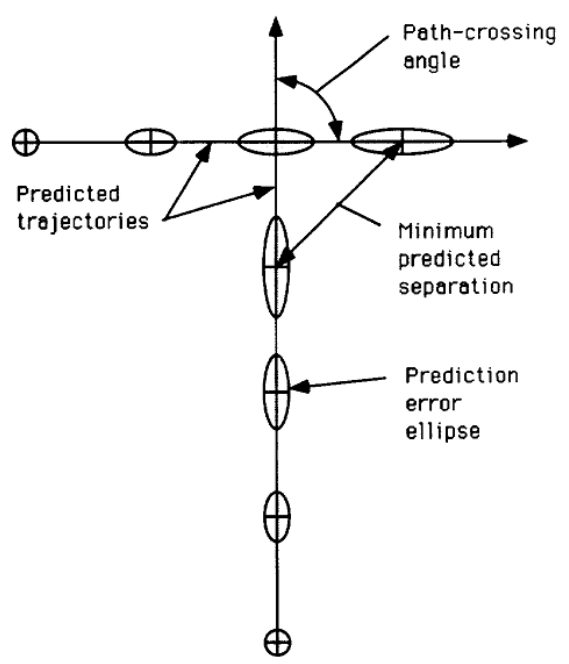

Figure 11 Prediction of position errors [Erzberger et al. 1997] 
One aircraft is treated as a stochastic aircraft whose trajectory prediction error is modeled as a normal distribution with zero mean. The other aircraft is the static aircraft. A covariance matrix is used to combine the trajectory prediction errors for both aircraft into a single distribution. The shape of the resulting prediction error is an ellipse. Then the overlap area of the conflict region with the prediction error is the conflict probability. An effective conflict resolution is to change the relative heading of the two aircraft so that the resulting conflict region is outside of the prediction error region.

Possible maneuvers in the flow corridor concept-of-operations are lane change maneuver and speed changes. The types of maneuver used in different circumstances depend on the predefined algorithm embedded in the onboard conflict detection and resolution system. As shown in section 2.3.1, the onboard conflict detection and resolution system collects the information of an aircraft pair that is on course for an NMAC such as target speed, position and time to first loss of separation. This information decides the priority of the conflict.

Some examples of studies that address conflict resolution algorithms for the AAC concept are the following. Erzberger [2006] discuss a set of preferred resolution and relative conflict maneuvers for different types of conflicts. High priority resolutions are those maneuvers that create less delay and deviate less from the original flight plan trajectory. The Resolution Maneuver Generator (RMG) is developed which contains algorithms that decide the coordinates of the return waypoint given a resolution maneuver. The resolution generator considers constrains such as airspace boundaries, turbulence, etc. Ezberger and Heere [2011] discuss an algorithm for resolving short range conflicts. The 
algorithm provides horizontal resolution trajectories considering limits on bank angle to achieve the required separation at the closest point of approach. Bank angle is emphasized as an important factor in the algorithm because, at short range, the finite turn rate of an aircraft must be considered. An example of the conflict resolution is to make one aircraft turn and the other to go straight. Takeichi et al. [2012] discuss a type of conflict resolution algorithm based on a relative velocity vector. The algorithm is applied on the flow corridor concept. The initial separation when an aircraft enters the flow corridor is equal to the required separation. The speed of each aircraft follows a uniform distribution between $230 \mathrm{~m} / \mathrm{s}$ and $250 \mathrm{~m} / \mathrm{s}$. The initial across-track positions and headings are also random variables. The resolution maneuver is to have both aircraft in conflict make turns to opposite directions. The results show that the algorithm can achieve the conflict free operation with a large traffic throughput.

\subsection{Traffic Collision Avoidance System}

TCAS provides airborne collision avoidance services at short range based on the processing of transponder replies from nearby aircraft. An onboard direction finding antenna is used to measure bearing of the intruder aircraft. Transponders (Mode S or Mode C) are interrogated to provide the position information of the aircraft. When two aircraft with close proximity are on course for an NMAC, TCAS generates traffic advisories and resolution advisories to pilots. The resolution advisory is displayed 20-30 seconds before the closest point of approach. The coordination algorithm assures that complementary resolution advisories increase the separation between the aircraft. For example, if one aircraft has a climb advisory, the other has a descend advisory. 
According to the description of the TCAS concept, it can be decomposed into several components, including the transponder, speaker, and other related components which refer to the resolution advisory generation and display system [Hemm and Busick 2009, Blum, et al. 2010].

Lebron et al. [1983] uses a fault tree analysis to estimate the failure rate of TCAS. The results are that pilot alertness is necessary in visual conditions. Pilots must respond to resolution advisories as soon as they are posted. Another study estimates the failure probability of TCAS by assuming that TCAS fails when any of the three components fail [Blum et al. 2010]. The three components are Mode S transponder, speaker, and other components excluding transponder and speaker. The Mode $\mathrm{S}$ transponder can be further decomposed into the TCAS processor, Mode $\mathrm{S}$ transponder, TCAS control panel and altitude indicator [Hemm et al. 2009]. The mean time between failures for each component gives the failure rate for each component. For example, the failure rate of the TCAS transponder is 9.7E-05 per hour. The failure rate of the speaker is assumed to be 1E-06 per hour. From the component failures, the overall failure probability of TCAS can be calculated.

\subsection{Safety Methods}

This section discusses some widely used safety analysis methodologies in the literature. Hazard analysis techniques are used to identify potential hazards for the system to be studied. For example, Preliminary Hazard Analysis (PHA) identifies hazardous conditions along with hazard causes, effects and corrective actions. Functional Hazard 
Analysis (FHA) assesses the effects of functional failures on the system. Finally Hazard and Operability Study (HazOp) considers human operations.

Fault tree analysis was developed by Bell Telephone Laboratories [Watson and Hill 1961] and has been widely used to analyze the causes of an event with serious consequences. The causes leading to the top of the fault tree are modeled through a graph where links between failures and/or operational error are Boolean operators, such as logic AND/OR gates.

Event tree analysis is used for modeling sequences of events given a trigger event. It can result in many different possible outcomes and the probability of each outcome is calculated. When modeling an entire system, event tree analysis can cover all different elements in the system such as subsystems, components, procedures and human behaviors. As a result, event tree analysis is good for conceptual design or top-level design of complex systems. It also works well with fault tree analysis which connects sequence of events with detailed components. A weakness of event tree analysis is that subtle system dependencies when modeling the events can be overlooked.

An extension of event tree analysis is dynamic event tree (DET) analysis. [Hofer et al. 2004]. The analysis is represented by a tree in which branching can occur at arbitrary discrete points in time. The time of the occurrence of branches is required by the system, thus creating a sequence of events based on the time of their occurrence. Within the timeframe of a function, the next branching may occur in different times but in the same state. The main advantage of the DET methodology over the conventional event 
tree method is that it simulates a probabilistic system evolution in a manner consistent with the conflict model.

Probabilistic risk models deal with sequence of events where time has an influence on the system behavior. For example, Monte Carlo simulation, discrete event simulations, dynamic event tree analysis and hybrid-state Markov processes are all techniques being used to model complex systems. Monte Carlo simulation is able to capture the statistical behavior of the system by including the intermediate state for events. Discrete event simulation is another simulation technique to model the sequence of events given the trigger conditions of events. However, unlike Monte Carlo simulations, it does not require expressions for the transition probabilities, but rather a "what-if" description of the system components. 


\section{CHAPTER 3 METHODOLOGY FOR COLLISION PROBABILITY ESTIMATION OF FLOW CORRIDORS}

This chapter discusses the overall methodology to estimate the collision probability of the flow corridor concept-of-operations. The chapter uses a hybrid method that combines Monte Carlo simulation and dynamic event tree analysis. Simulation and dynamic event trees are two widely used methods to model complex systems. However, collision probabilities are difficult to estimate with standard Monte Carlo simulation because the occurrence rates are so low (e.g., 1E-9 collisions per flight hour). The computer time required to obtain a reasonable estimate may be prohibitive. (For example, it would take about 3 years to obtain an estimate for a 1 in $10^{9}$ event, with a $10 \%$ relative error, assuming 1,000 replications per second.) On the other hand, dynamic event tree analysis itself cannot capture the detailed stochastic behavior of the aircraft (e.g., position and speed as a function of time). For example, it cannot model the dependent relationship between the stochastic variables in the flow corridor (e.g., lane changes maneuvers due to traffic congestion).

As a result, the collision probability is decomposed into two parts. Simulation is used to capture the statistical behavior of aircraft by including the intermediate state such as separation and relative speed. The simulation models the aircraft performing self separation and lane change maneuvers. The objective of the simulation is to estimate the rates of potential conflicts (which are not very rare). The dynamic event tree is then used 
to model the automated separation assurance system given that two aircraft are on course for an NMAC. The automated separation assurance system contains four levels of conflict detection and resolution functions to prevent collision.

The rest of this chapter is organized as follows: Section 3.1 identifies a list of hazards that can occur in the flow corridor operations. This includes functional failures and operational errors. Section 3.2 discusses the hybrid methodology to estimate the collision probability of the flow corridor concept-of-operations.

\subsection{Hazard Analysis}

This section gives a preliminary hazard list for the flow corridor concept. Hazards were obtained through brainstorming sessions and related articles on AFM. Various references on AFM were used as a foundation for identifying hazards [Wing et al. 2010]. Many of the hazards identified in this section are also given in these papers. The hazards are grouped into the following functional categories:

- Navigation

- Communication

- Surveillance

- Conflict detection and resolution

- Operational error 
Table 5 Hazards in flow corridor operations

\begin{tabular}{|c|c|c|c|c|}
\hline Category & Hazard & Consequence & Reference & $\begin{array}{c}\text { Modeled in } \\
\text { the } \\
\text { dissertation }\end{array}$ \\
\hline \multirow[b]{2}{*}{ Surveillance } & ADS-B fails & $\begin{array}{c}\text { ADS-B aircraft lose } \\
\text { awareness }\end{array}$ & \multirow[b]{2}{*}{$\begin{array}{l}\text { [Wing and } \\
\text { Cotton } \\
\text { 2011] }\end{array}$} & Yes \\
\hline & $\begin{array}{c}\text { Radar } \\
\text { surveillance fails }\end{array}$ & $\begin{array}{l}\text { ADS-B aircraft lose } \\
\text { awareness of non- } \\
\text { ADS-B aircraft }\end{array}$ & & Yes \\
\hline \multirow{4}{*}{ Navigation } & $\begin{array}{l}\text { GPS satellites } \\
\text { fails }\end{array}$ & $\begin{array}{c}\text { Reliability of } \\
\text { navigation system } \\
\text { downgrades }\end{array}$ & \multirow{4}{*}{$\begin{array}{l}\text { [Wing and } \\
\text { Cotton } \\
\text { 2011] }\end{array}$} & Yes \\
\hline & $\begin{array}{l}\text { GPS receiver } \\
\text { fails }\end{array}$ & $\begin{array}{l}\text { Reliability of } \\
\text { navigation system } \\
\text { downgrades }\end{array}$ & & Yes \\
\hline & $\begin{array}{l}\text { Radio navigation } \\
\text { system fails }\end{array}$ & $\begin{array}{c}\text { Reliability of } \\
\text { navigation system } \\
\text { downgrades }\end{array}$ & & Yes \\
\hline & $\begin{array}{c}\text { Inertial } \\
\text { navigation } \\
\text { system fails }\end{array}$ & $\begin{array}{c}\text { Reliability of } \\
\text { navigation system } \\
\text { downgrades }\end{array}$ & & Yes \\
\hline \multirow[t]{2}{*}{ Communication } & CPDLC fails & $\begin{array}{c}\text { Controllers are } \\
\text { unable to send } \\
\text { commands via data } \\
\text { link such as corridor } \\
\text { shift }\end{array}$ & \multirow[t]{2}{*}{$\begin{array}{l}\text { [Blum et } \\
\text { al. 2010] }\end{array}$} & No \\
\hline & $\begin{array}{c}\text { Voice } \\
\text { communication is } \\
\text { down }\end{array}$ & $\begin{array}{l}\text { Pilots are unable to } \\
\text { receive audio } \\
\text { conflict resolution }\end{array}$ & & Yes \\
\hline Display & CDTI fails & $\begin{array}{l}\text { Pilots are unable to } \\
\text { locate their own } \\
\text { position. Controllers } \\
\text { are responsible to } \\
\text { maintain separation }\end{array}$ & \multirow{2}{*}{$\begin{array}{l}\text { [Blum et } \\
\text { al. 2010] }\end{array}$} & Yes \\
\hline \multirow[b]{2}{*}{ Conflict CD\&R } & $\begin{array}{l}\text { Conflict CD\&R } \\
\text { fail to provide } \\
\text { resolutions } \\
\text { (hardware failure } \\
\text { or algorithm } \\
\text { flaw) }\end{array}$ & $\begin{array}{l}\text { Dependent on pilots } \\
\text { situation awareness, } \\
\text { may cause conflict }\end{array}$ & & Yes \\
\hline & $\begin{array}{l}\text { Conflict CD\&R } \\
\text { provide a } \\
\text { resolution that }\end{array}$ & $\begin{array}{c}\text { A secondary } \\
\text { conflict occurs }\end{array}$ & & No \\
\hline
\end{tabular}




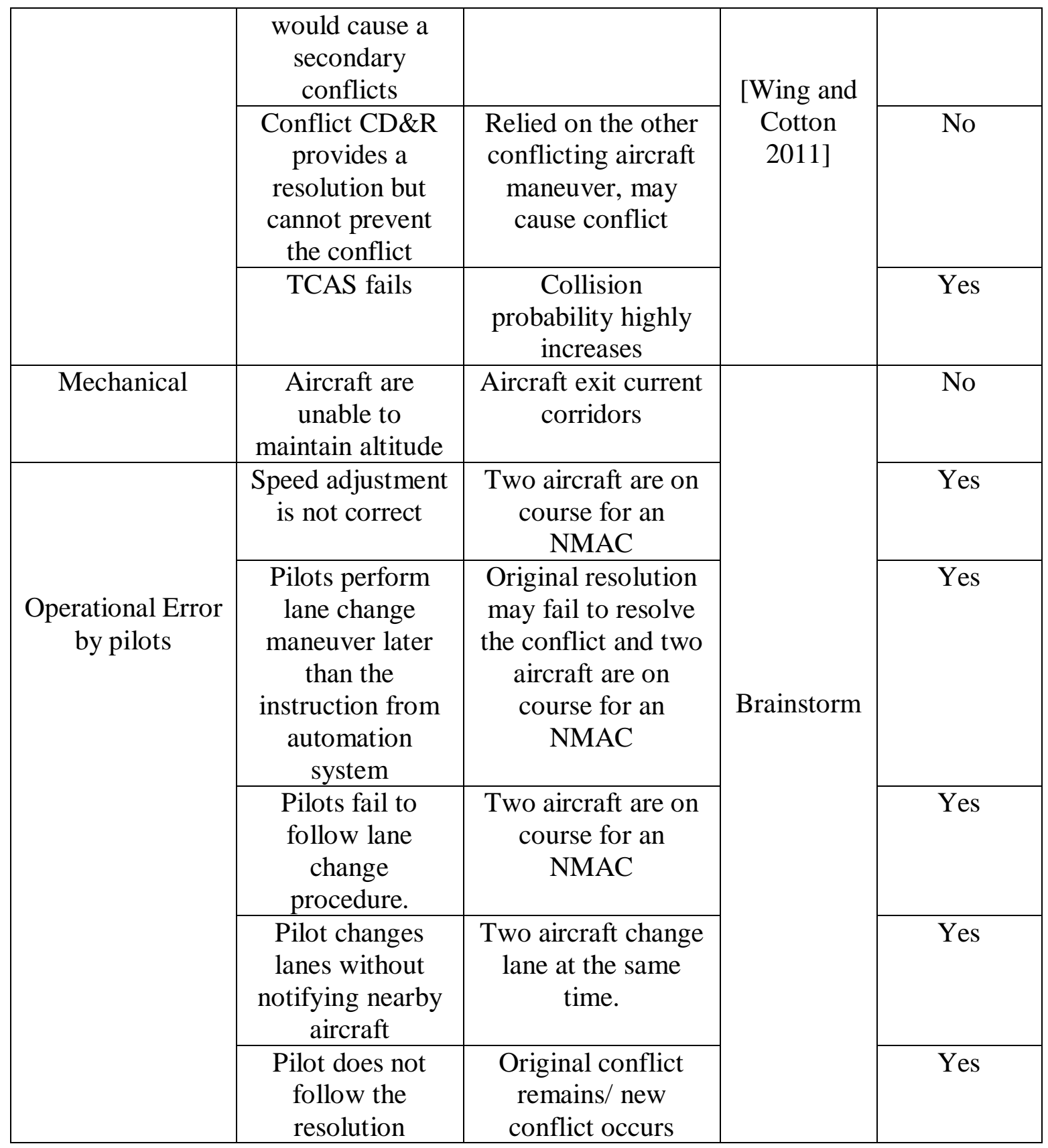

The hazard analysis provides a guideline for the collision analysis. It lists things that can go wrong. Most of the hazards are analyzed in the dissertation. Several hazards are not analyzed. For example, secondary conflicts are not analyzed because it is assumed 
that a conflict resolution, if successfully executed, is conflict free and does not lead to downstream secondary conflicts.

\subsection{Methodology and Framework to Estimate Collision Probability}

This section describes the overall methodology and frame work used to estimate

the collision probability of the flow corridor concept-of-operations. The method is based on decomposing collision events into pre-cursor scenarios and then evaluating the individual probabilities of those scenarios. Figure 12 shows the pre-cursor scenarios. In this paper, a collision is defined as an event in which the horizontal separation between two aircraft is less than 100 feet. An NMAC is defined as an event in which the horizontal separation between two aircraft is less than 500 feet [Kuchar et al. 2004]. The pre-cursor scenarios are: 1) Before a collision occurs, an NMAC occurs; 2) before an NMAC occurs, two aircraft are on course for an NMAC (an NMAC trajectory); and 3) before two aircraft are on course for an NMAC, they are located at positions that can result in being on course for an NMAC if a blunder occurs. We refer to this latter situation as a potential NMAC trajectory. This concept will be described more carefully in the next section. 


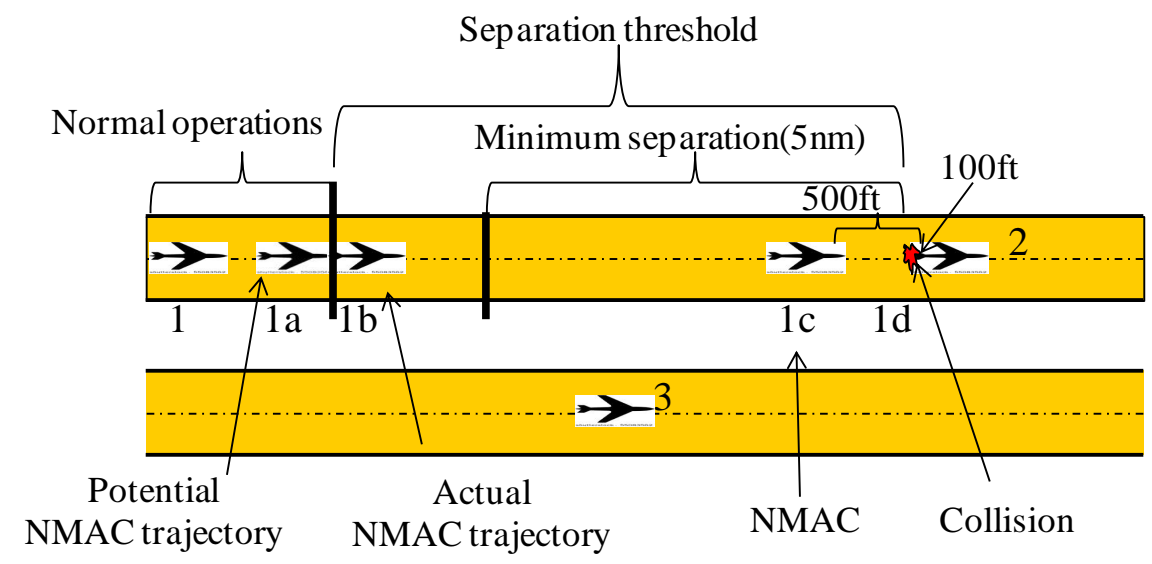

Figure 12 Pre-cursor scenarios to collision

Figure 13 shows four different geometric scenarios in which a collision may occur between two aircraft in the flow corridor (assuming two parallel lanes at the same altitude). 1) Overtaking scenario: the trailing aircraft with greater velocity collides with the leading aircraft in the same lane. 2) Lane change scenario: one aircraft switches lanes resulting in a collision with another aircraft in the adjacent lane. 3) Simultaneous lane change scenario: two aircraft in separate lanes switch lanes almost at the same time and collide in the middle of maneuvers. 4) Lane change and overtaking scenario: one aircraft switches lanes resulting in loss of separation with another aircraft in the adjacent lane and then collides via overtaking (alternatively, if the aircraft switching lanes ends up in front of the aircraft in the adjacent lane, then the collision could occur as a result of overtaking by the trailing aircraft in the adjacent lane). 


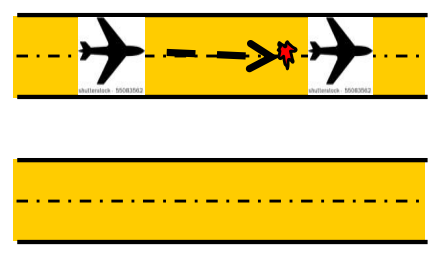

1. Overtaking

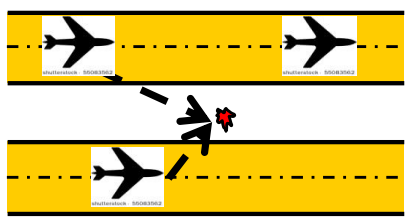

3. Simultaneous lane change

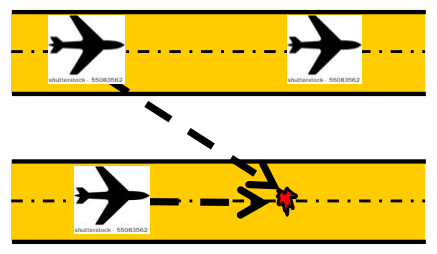

2. Lane change

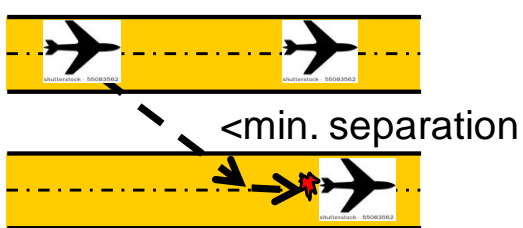

4. Lane change and overtaking

Figure 13 Scenarios lead to collision

Based on the laws of probability theory, the overall probability of a collision can then be written as follows:

$$
\begin{aligned}
& \text { Equation } 3 \text { Collision probability } \\
& \qquad \begin{aligned}
\operatorname{Pr}\{\text { collision }\} & =\sum_{i} \operatorname{Pr}\{\text { potental NMAC trajectory } i\} \\
& * \operatorname{Pr}\{\text { NMAC trajectory } i \mid \text { potential NMAC trajectory } i\} \\
& * \operatorname{Pr}\{\text { collision |potential NMAC trajectory } i \text {, NMAC trajectory } i\}
\end{aligned}
\end{aligned}
$$

where $i(=1,2,3,4)$ corresponds to one of the four scenarios in Figure 13. Since the probabilities of occurrence of the potential NMAC trajectory are not extremely rare (compared to the probability of an actual collision), simulation can be used to estimate 
the occurrence rates. The list below summarizes how each element in Equation 3 is calculated.

- $\operatorname{Pr}\{$ potential NMAC trajectory $i\}$ : This probability is calculated via a Monte-Carlo simulation of the flow corridor concept-of-operations (Chapter 4). Potential NMAC trajectory occurs at certain decision points in the normal operations where an erroneous decision made can result in an NMAC on course. For example, a potential-NMAC-trajectory-1 event (or potential overtaking-event) occurs at the decision point when a faster trailing aircraft must decide to either slow down or switch lanes to avoid overtaking or subsequent loss of separation. At this point, if the trailing aircraft does not see the leading aircraft, then the trailing aircraft will continue overtaking and will subsequently be on course for an NMAC.

- $\operatorname{Pr}\{$ NMAC trajectory $i \mid$ potential NMAC trajectory $i\}$ : This is the probability that a potential NMAC trajectory results in an actual NMAC trajectory as a result of a blunder or function failure. For example, referring to the overtaking scenario, at the decision point to slow down or change lanes, the potential NMAC trajectory becomes an actual NMAC trajectory (that is, two aircraft on course for an NMAC) if the trailing aircraft cannot locate itself or the leading aircraft (loss of locatability). The failure probability is calculated using reliability diagrams as described in Chapter 5.

- $\operatorname{Pr}\{$ collision $\mid$ NMAC trajectory $i$, potential NMAC trajectory $i\}$ : When two aircraft are on course for an NMAC, the CD\&R functions provide safety layers to prevent the impending NMAC or possible collision. The CD\&R process is 
modeled via dynamic event trees. Dynamic event tree analysis is an analytical technique that models the process as a tree in which branching can occur at arbitrary discrete points in time. Reliability diagrams are linked to the dynamic event trees to decompose CD\&R functions into hardware components. Detailed analysis is discussed in Chapter 5.

The overall collision probability is the summation of conditional collision probabilities for each event.

\subsection{Summary}

A hybrid methodology is developed to estimate the collision probability of the flow corridor concept-of-operations. The methodology is based on decomposing collision events into four pre-cursor scenarios and then evaluating the individual probabilities of those scenarios. The probability of each pre-cursor scenario is further decomposed based on the level of risks. The probabilities of the potential NMAC trajectory are calculated via a Monte-Carlo simulation of the flow corridor concept-of-operations to estimate the occurrence rates. The flow corridor simulation is discussed in Chapter 4. The probability that a potential NMAC trajectory results in an actual NMAC trajectory is modeled via reliability diagrams as discussed in Chapter 5. The probability of a collision given an actual NMAC trajectory depends on the performance of the CD\&R functions. The $\mathrm{CD} \& \mathrm{R}$ process is modeled via dynamic event trees, discussed in Chapter 5. 


\section{CHAPTER 4 FLOW CORRIDOR SIMULATION}

The goal of the flow-corridor simulation is to model speed adjustment and lanechange maneuvers of aircraft within the corridor in order to provide the occurrence rates of potential NMAC trajectories, which are related to the level of congestion within the corridor. In the simulation, it is assumed that aircraft strictly follow the rules of selfseparation so that no actual conflicts or collisions occur. Section 4.1 discusses inputs of the flow corridor simulation. Section 4.2 illustrates the basic flow logic of the simulation. Section 4.3 discusses the outputs of the flow corridor simulation. Section 4.4 summarizes the assumption made in the simulation. Section 4.5 discusses experiments to verify the correctness of the flow corridor simulation.

\subsection{Simulation Inputs}

The simulation inputs can be categorized into four types: Corridor-geometry parameters, aircraft parameters, separation parameters and arrival process parameters.

\subsubsection{Corridor-Geometry Parameters}

Figure 14 shows the basic corridor geometry assumed in this research. The

parameters are as follows:

- Lanes: The corridor consists of 2 parallel straight lanes. This is the simplest corridor geometry. 
- Length: The length of each lane is 600 nautical miles. This could connect city pairs like New York City and Chicago.

- Width: The width of each lane is 4 nautical miles. This is consistent with RNP-1 capability in which aircraft are required to fly within \pm 2 nautical miles of the centerline $99.995 \%$ of time.

- Altitude: The altitude of both lanes is 35,000 feet. This is a typical en-route altitude.

- Lane separation: The cross-track distance between the centerlines of the two lanes is 8 nautical miles.

- Lane change angle: The angle between the lane-change maneuver and the center line of the original lane is 30 degrees. This is an assumed regulation that aircraft need to follow.

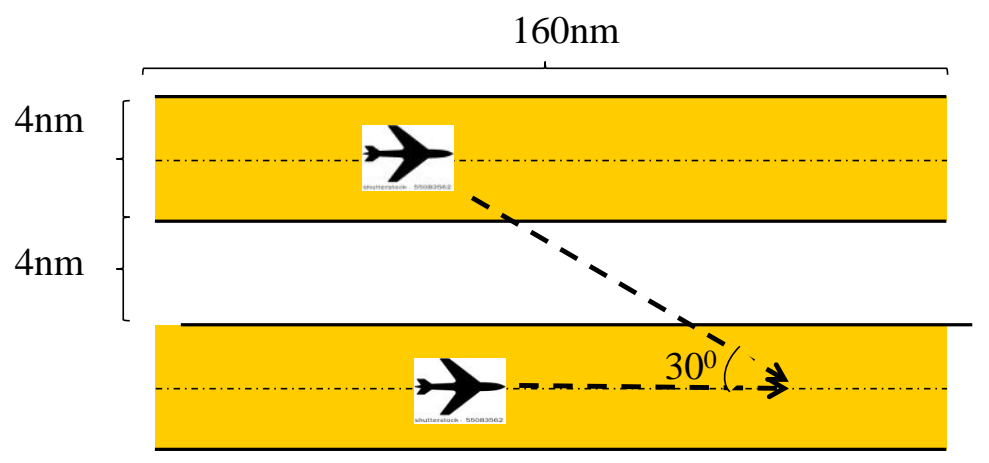

Figure 14 Example of the flow corridor model 


\subsubsection{Aircraft Parameters}

Aircraft parameters include the basic physical characteristics of each type of

aircraft. The values of the parameters are from the Base of Aircraft Data [BADA 2013].

- Maximum speed: This is the highest speed that an aircraft can fly. Each type of aircraft has its own maximum speed.

- Stall speed: This is the lowest speed at which an aircraft can maintain level flight. An aircraft flying at its stall speed cannot climb, and an aircraft flying below its stall speed cannot stop descending.

- Maximum altitude: This is the highest altitude that an aircraft can fly.

\subsubsection{Arrival-Process Parameters}

The arrival-process parameters determine the throughput and congestion of the flow corridor as well as the frequency of occurrence of potential events that lead to a collision.

- Fleet mix: The fleet mix is quantified by the percentage of each aircraft type in the flow corridor. When an aircraft enters the corridor, its type is randomly generated from a discrete probability distribution in an independent and identically distributed manner. Unless stated otherwise, the baseline fleet mix consists of two aircraft types, B737 and B742.

- Target velocity: This is the velocity that an aircraft would like to fly in the flow corridor. Let $T_{i}$ be the target velocity of an arbitrary aircraft of type $i . T_{i}$ is assumed to follow a normal distribution. The target velocity is randomly chosen for an aircraft when it enters the corridor, but it is fixed for that aircraft as it traverses the corridor. 
- Initial separation: This is the initial separation between successive aircraft in the same lane as they enter the corridor. The initial separation equals the minimum separation plus a separation buffer plus a random variable that follows an exponential distribution with a baseline mean of 1 nautical mile.

\subsubsection{Separation Parameters}

Separation parameters determine the assumed separation rules that aircraft need to follow in the flow corridor.

- Minimum separation: In current operations, the required minimum lateral separation between aircraft is 5 nautical miles. In the flow corridor, it may be reduced.

- Separation buffer: This is an extra separation buffer that is applied to the minimum separation requirement. The baseline value is 20 percent of the minimum separation. For example, if the minimum separation is 5 nautical miles, then the added buffer is 1 nautical mile.

- Separation threshold: This is the summation of the minimum separation and the separation buffer. For example, if the minimum separation is 5 nautical miles and the separation buffer is 1 nautical mile, then aircraft are required to maintain 6 nautical mile separation with each other.

- Relative velocity threshold: This value is used to determine whether or not the trailing aircraft attempts to pass a slower aircraft. If the relative velocity between the trailing aircraft and the leading aircraft is smaller than the threshold, the 
trailing aircraft does not attempt to change lanes, but rather reduces its speed to follow the leading aircraft. If the relative velocity is greater than the threshold, the trailing aircraft attempts to switch lanes if possible.

\subsubsection{Parameters Summary}

Table 6 Simulation parameters

\begin{tabular}{|c|c|c|}
\hline Parameters & Baseline value & Varied in the simulation \\
\hline Lanes & 2 & No \\
\hline Length of the lane & $600 \mathrm{~nm}$ & No \\
\hline Width of the lane & $4 \mathrm{~nm}$ & No \\
\hline Altitude of the lane & $35000 \mathrm{ft}$ & No \\
\hline Lane separation & $4 \mathrm{~nm}$ & No \\
\hline Lane change angle & 30 degrees & No \\
\hline Maximum velocity & $0.82 \mathrm{mach}$ & Yes \\
\hline Minimum separation & $5 \mathrm{~nm}$ & Yes \\
\hline Separation buffer & $1 \mathrm{~nm}$ & Yes \\
\hline Separation threshold & $6 \mathrm{~nm}$ & Yes \\
\hline Relative velocity threshold & $15 \mathrm{knots}$ & Yes \\
\hline Fleet mix & $(0.2,0.8)$ & Yes \\
\hline Target Velocity & Norm $\left(350 \mathrm{knots}, 5^{2}\right)$ & Yes \\
\hline Initial separation & $\operatorname{Exp}(7.5 \mathrm{~nm})$ & \\
\hline
\end{tabular}

\subsection{Flow Logic of Simulation}

The goal of the simulation is to model speed-adjustment and lane-change

maneuvers within the corridor. It is assumed that aircraft strictly follow the rules of selfseparation so that no actual conflicts or collisions occur within the simulation. Ye et al. [2014] have done similar work on modeling lane-change maneuvers in flow corridors. The main contribution here is to estimate the rate of potential NMAC trajectories from the simulation for use in the overall calculation of the collision probability. 
Figure 15 shows the main logic used in the simulation. For each aircraft in the corridor, the separation with its leading aircraft is calculated. If the separation is larger than the separation threshold (the minimum separation plus the separation buffer), the trailing aircraft flies its target velocity. An aircraft without a leading aircraft also flies its target velocity. If the separation is smaller than the separation threshold, the simulation checks the relative velocity between the trailing aircraft and the leading aircraft. If the relative velocity is smaller than the relative velocity threshold, the trailing aircraft reduces its velocity and follows the leading aircraft at the exact speed of the leading aircraft. It is assumed that the trailing aircraft can reduce its speed to that of the leading aircraft in one time step. If the relative velocity is larger than the threshold, the trailing aircraft checks if there is an opening to change lanes. If there is an opening, the aircraft performs a lane change maneuver. If not, it reduces its speed to that of the leading aircraft and continues to monitor the opposite lane for availability. These steps are repeated at successive time increments, where the time step is 6 seconds. 


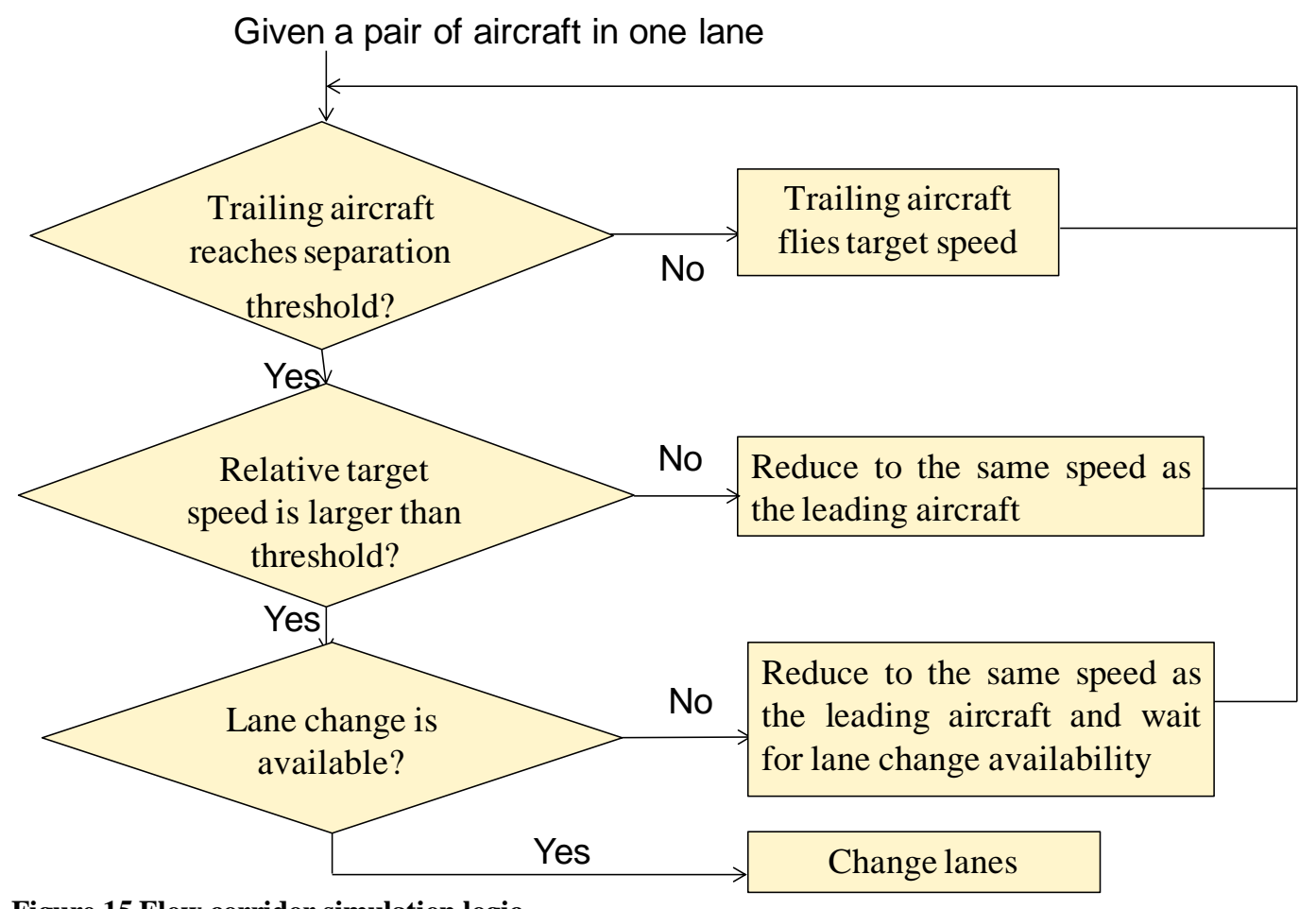

Figure 15 Flow corridor simulation logic

To determine the availability of lane change maneuvers, there are three requirements. Figure 16 shows an example of four aircraft in which aircraft 1 would like to pass aircraft 2. First, the velocity of aircraft 4 must be larger than the velocity of aircraft 2, because otherwise there is no benefit to changing lanes. Second, the projected separation $x_{13}$ between aircraft 1 and aircraft 3 (after the lane change maneuver is executed) must be larger than the required separation, the minimum separation plus the separation buffer. Finally, the projected separation $x_{14}$ between aircraft 1 and aircraft 4 must be larger than the required separation as well. 


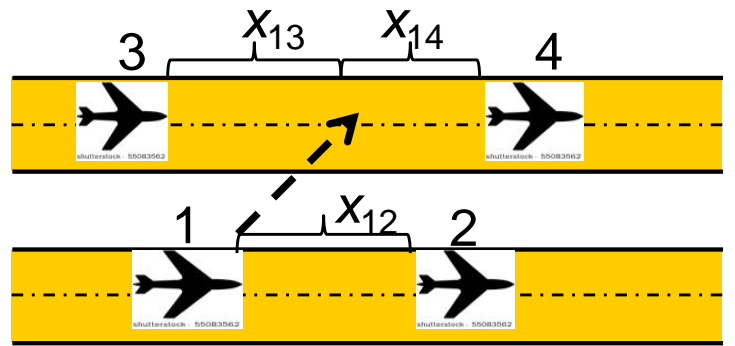

Figure 16 Example of lane change maneuver

\subsection{Simulation Outputs}

The outputs of the simulation are the frequencies of potential NMAC trajectory).

No actual conflicts, NMACs, or collisions occur in the simulation. Rather, the simulation counts events such that - if there were a failure - the event would lead to an NMAC trajectory. The following discussion defines precisely how these potential events are counted in the simulation.

Potential NMAC trajectory 1 (overtaking): The event occurs at the point when the following aircraft must reduce speed or change lanes to avoid a subsequent loss of separation. This occurs (see Figure 16 错误！未找到引用源。) when

1) $x_{12} \leq$ minimum separation + separation buffer

2) $v_{1}>v_{2}$, where $v_{i}$ is the velocity of aircraft $i$.

Potential NMAC trajectory 2 (lane change): This event occurs when aircraft 1 would like to change lanes and the projected separation with aircraft 3 or aircraft 4 is less than $500 \mathrm{ft}$. (Thus, if aircraft 1 fails to see the aircraft in the other lane, then executing the desired lane change would result in an NMAC). This occurs when: 
1) $x_{12} \leq$ minimum separation + separation buffer, $v_{1}-v_{2}>$ relative velocity threshold, $v_{4}>v_{2}$ (aircraft 1 would like to change lanes).

2) $x_{13}<500$ feet or $x_{14}<500$ feet.

The occurrence of this event can only be counted once, at the first instance when the in-trail separation is less than or equal to the separation threshold. The rationale is that the aircraft only needs to "see" the other aircraft at the initial decision point to switch lanes. If a lane change is unavailable, the aircraft is assumed to "remember" the presence of the other aircraft until a lane change becomes available.

Potential NMAC trajectory 3 (simultaneous lane change): This event occurs when aircraft 1 would like to change lanes and aircraft 3 is faster than aircraft 4. (Both aircraft reach the separation threshold at the same time step.) The projected separation between aircraft 1 and aircraft 4 is less than $500 \mathrm{ft}$. (The symmetric case counts as another event.) This occurs when

1) $x_{12} \leq$ minimum separation + separation buffer and $v_{1}-v_{2}>$ relative velocity threshold $v_{4}>v_{2}$ (aircraft 1 would like to change lanes).

2) $x_{34} \leq$ minimum separation + separation buffer and $v_{3}-v_{4}>$ relative velocity threshold

3) $0.5 * x_{13}<500$ feet

Potential NMAC trajectory 4 (lane change and overtaking): This event occurs when aircraft 1 would like to change lanes, the projected separation with aircraft 3 (or aircraft 4) is less than the required separation, and aircraft 1 is faster than aircraft 3 . For example, 
1) $x_{12} \leq$ minimum separation + separation buffer and $v_{1}-v_{2}>$ relative velocity threshold and $v_{4}>v_{2}$.

2) $x_{13}<$ minimum separation and $x_{13} \geq 500 \mathrm{ft}$, or $x_{14}<$ Minimum separation and $x_{14} \geq$ $500 \mathrm{ft}$

3) $v_{1}>v_{4}$

\subsection{Simulation Verification}

This section discusses an experiment designed to verify the correctness of the flow corridor simulation. Figure 17 shows an analytical conflict rate model from Siddiqee [1973]. There are two lanes intersecting at point $\mathrm{P}$ with angle $\alpha$. Lane $\mathrm{AB}$ has a flow rate $f_{1}$ (aircraft / hour). Lane CD has a flow rate $f_{2}$. A conflict is assumed to occur if the distance between the two aircraft, when one is at $\mathrm{P}$, is less than or equal to $w$. Each aircraft is assumed to have a constant velocity $v$.

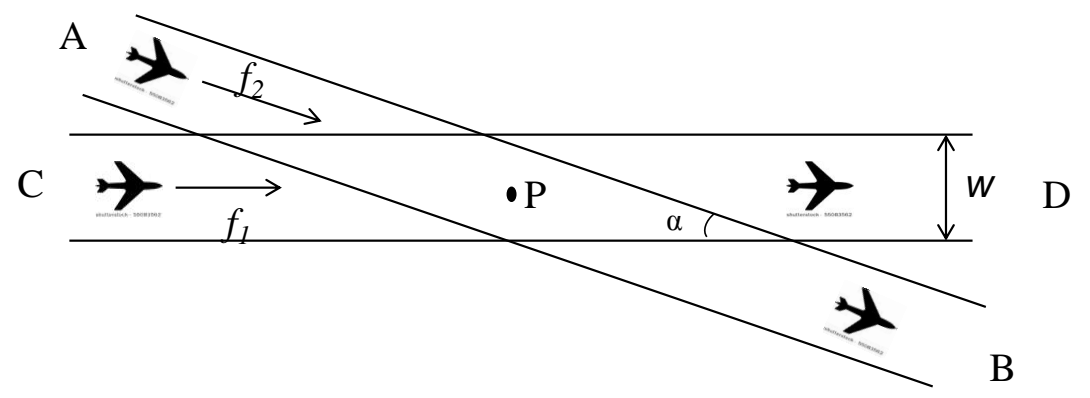

Figure 17 A conflict rate model

The rate of conflicts is found analytically to be [Siddiqee 1973]: 
Conflict rate $=\mathrm{f}_{1} * \mathrm{f}_{2} \frac{2 * \mathrm{NMAC}+\mathrm{w} * \tan (\alpha / 2)}{\mathrm{v}}$

where $\mathrm{NMAC}=500 \mathrm{ft}$

In the flow corridor simulation, the two lanes are parallel. However, the lanechange flight path can be viewed as a crossing lane like CD in Figure 17. A simple simulation experiment is designed to mimic the crossing pattern and verify that the resulting number of conflicts matches the previous equation. In this experiment (Figure 18), the following assumptions are made:

1) All aircraft in lane 2 have the same velocity of 360 knots.

2) The velocity of an aircraft in lane 1 is randomly chosen as either 350 knots or 360 knots (equal chance for each case).

3) The relative velocity requirement for the lane change maneuver is set at less than 10 knots so that all aircraft with a velocity of 360 knots will eventually want to change lanes.

4) The initial separation between aircraft in lane 1 is a fixed value of 7 nautical miles. The initial separation between aircraft in lane 2 is also fixed, but it is increased by 0.5 nautical miles for each simulation experiment. 


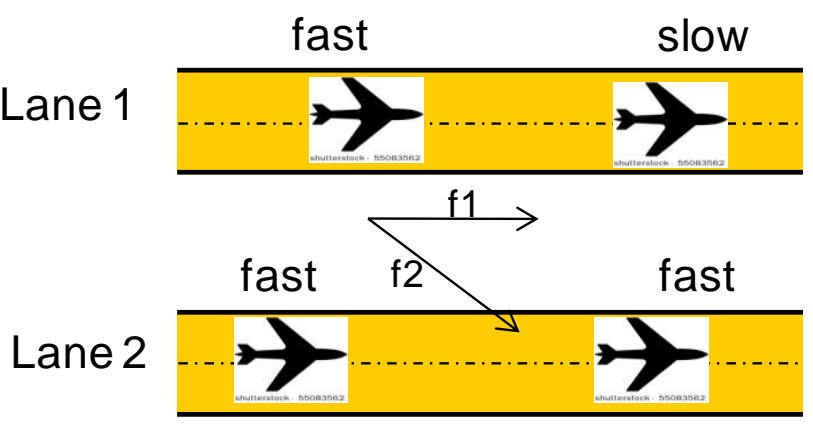

Figure 18 Simulation verification experiment

Comparing to the analytical model, the flow rate of lane 1 is $\frac{360}{7}$ flights per hour and the flow rate of the lane change maneuver is $\frac{360}{14}$ flights per hour. The conflict area is NMAC which is 500 feet (0.08 nautical miles) in length and 500 feet in width. The crossing angle is 30 degrees. Thus, the rate of NMACs is estimated to be:

$$
P(C)=\frac{360}{7} * \frac{360}{14} * \frac{2 * 0.08+0.08 * \tan \left(\frac{30}{2}\right)}{\mathrm{V}}=0.67
$$

Figure 19 shows the conflict rates from the simulation and the analytical equation by varying the flow rate. They are consistent with each other. 


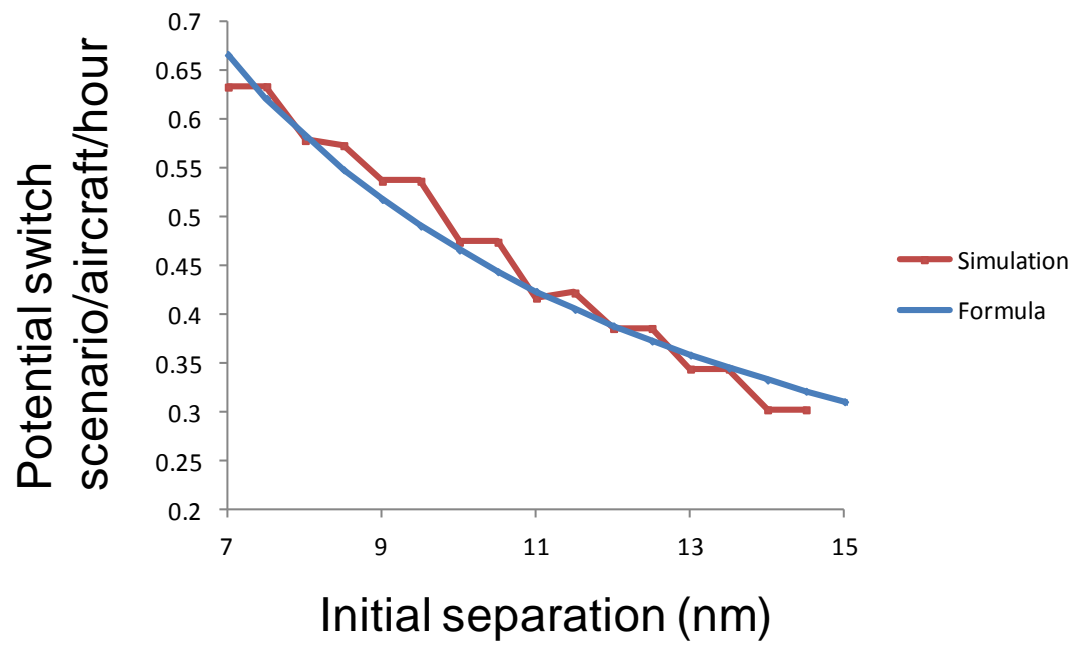

Figure 19 Result of simulation verification 


\section{CHAPTER 5 DYNAMIC EVENT TREE AND FAULT TREE ANALYSIS}

The simulation analysis in the previous chapter gives the rate of potential NMAC trajectories - that is, events in which two aircraft could be on course for an NMAC if a blunder were to occur. This chapter estimates the probability that a potential NMAC trajectory becomes an actual NMAC trajectory and then the probability that an actual NMAC trajectory becomes a collision. Two events that can cause an actual NMAC trajectory are considered: a loss of locatability (a functional failure of one aircraft to see another) and a loss of situational awareness (pilot error). A collision then may result from the failure of the automated separation assurance system. Dynamic event trees are used to model the automated separation assurance system. Reliability diagrams are linked to the dynamic event trees to provide failure probabilities of the CD\&R functions in the automated separation assurance system. The output of the dynamic event tree is the conditional collision probability of a collision scenario.

Section 5.1 gives a list of assumptions made for the analytical method. Section 5.2 discusses events that cause potential NMAC trajectories to become actual NMAC trajectories. Section 5.3 discusses sequences of events (i.e. the failure of CD\&R functions) that cause actual NMAC trajectories to become collisions. Section 5.4 gives sample calculations of the dynamic event trees and reliability diagrams. 


\subsection{Analysis Assumptions}

The methodology has the following assumptions:

- Component failures are independent of each other and identically distributed across flights. Component failures occur at the beginning of the time horizon of the event tree. Failed components remain failed throughout the time horizon of the event tree.

- CD\&R functions work in different time frames (strategic intent-based, tactical intent-based and tactical state-based). The model moves to the next time frame if (a) the CD\&R function fails to detect the conflict in the current time frame, or (b) the CD\&R function fails to provide resolutions in the current time frame, or (c) pilots do not execute the conflict resolution in the current time frame.

- In each time frame, pilots have different response time intervals to execute resolution maneuvers.

- The probability of detecting a conflict depends on the look-ahead time and the conflict geometry.

- If a conflict resolution is executed correctly by a pilot, the conflict is successfully resolved. No downstream secondary conflicts are considered.

- When an aircraft pair is on course for an NMAC in the strategic time frame, the aircraft not flying its flight plan is responsible for resolving the conflict. For the overtaking case, the following aircraft is responsible for resolving the conflict. The case studies have the following assumptions: 
- $\quad$ Aircraft are locatable either via ADS-B or TIS-B. The ADS-B in transponder is used by both the ADS-B and TIS-B systems. TCAS has a separate transponder to locate aircraft.

- The strategic and tactical intent-based conflict resolutions rely on the FMS, but the tactical state-based conflict resolutions do not.

- Strategic intent-based conflict resolutions are communicated via the navigation display.

- Tactical conflict resolutions are communicated via either the speaker or the primary flight display.

- The probability of a pilot blunder is independent of the calculation of the dynamic event tree.

\subsection{Events Leading to Actual NMAC Trajectory}

This section considers two events that cause potential NMAC trajectories to become actual NMAC trajectories: the failure of one aircraft to see another aircraft (loss of locatability) and the loss of situational awareness by pilot (pilot blunder). These events are modeled via reliability diagrams. Other events that might cause NMAC trajectories, such as a pilot exiting the corridor by mistake, are not considered.

\subsubsection{Loss of Locatability}

When the aircraft loses the ability to locate itself or other nearby aircraft, this is referred to as a loss of locatability (LOL). The locatability function is a critical part of CD\&R functions. Figure 20 shows the reliability diagram of the locatability function for one aircraft. In the figure, each box represents a component or set of components. The 
function works if there is at least one path through the diagram (from left to right) with all components working. The top path represents the ADS-B system and the bottom path represents the Traffic Information Service Broadcast (TIS-B) system. In the top path, the navigation system on each aircraft receives its own state information (position and velocity) via a Global Navigation Satellite System such as GPS and via an inertial navigation system. Aircraft 2 broadcasts its information via ADS-B out. Aircraft 1 receives the state information from aircraft 2 via ADS-B in ([Wing and Cotton 2011], p.39). In the lower path, TIS-B is a radar system that sends state information of all aircraft within a certain region (Wing and Cotton 2011], p.40]). Aircraft 1 receives this state information via the ADS-B in transponder. As a conservative assumption, it is assumed that if the locatability function fails for aircraft 1 (that is, aircraft 1 cannot locate either itself and/or aircraft 2 via Equation 4), then the locatability function also fails for aircraft 2 (that is, aircraft 2 cannot locate either itself and/or aircraft 1).

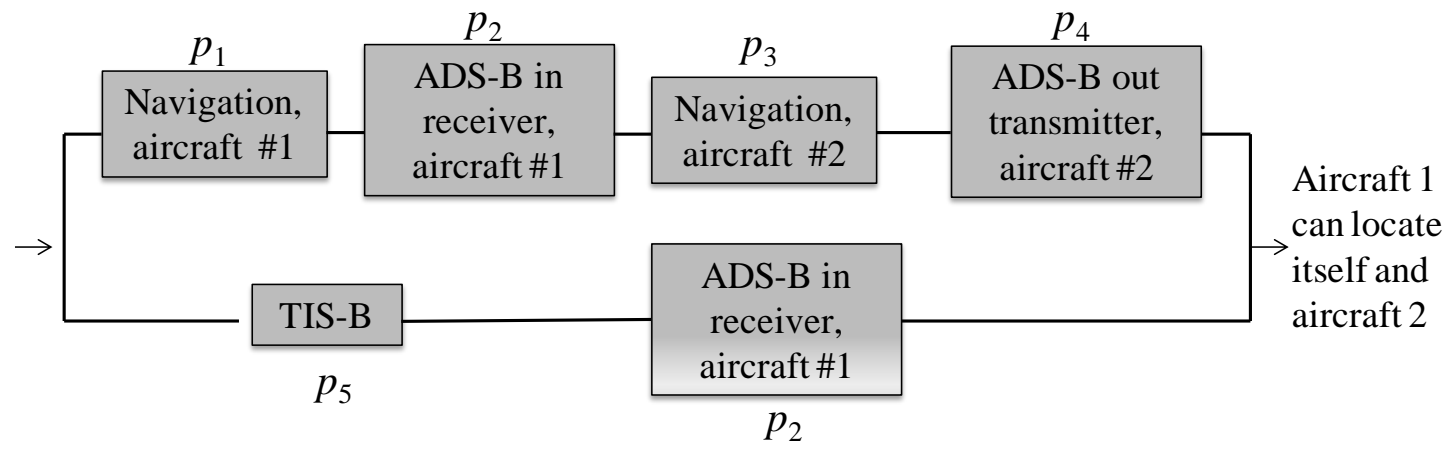

Figure 20 Reliability diagram of locatability function 
Let $p_{i}$ be the failure probability of component $i$. The complete list of components is given in the Appendix. From the reliability diagram,

$$
\begin{aligned}
& \text { Equation } 4 \text { Probability of loss of locatability } \\
& \quad \operatorname{Pr}\{\text { loss of locatability }\}=1-\left[\left(1-p_{1}\right)\left(1-p_{2}\right)\left(1-p_{3}\right)\left(1-p_{4}\right)+\right. \\
& \left.\left(1-p_{2}\right)\left(1-p_{5}\right)-\left(1-p_{1}\right)\left(1-p_{2}\right)\left(1-p_{3}\right)\left(1-p_{4}\right)\left(1-p_{5}\right)\right]
\end{aligned}
$$

\subsubsection{Pilot Blunder}

A loss of situational awareness can cause different types of NMAC trajectories.

For example, if the conflict resolution suggests slowing down to avoid overtaking, the NMAC trajectory is assumed to occur if the pilot loses situational awareness and does not execute the resolution. If the conflict resolution suggests staying in lane and waiting for lane change availability, an NMAC trajectory can also occur if the pilot executes a lane change maneuver before it is available. The probability of such an erroneous decision is assumed to be $1.5 \mathrm{E}-5$ based on the historical data of pilot deviation, which is defined as the actions of a pilot resulting in the violation of a federal aviation regulation [FAA 2012].

\subsection{Event Sequences Leading to Collisions}

This section discusses event sequences that lead from actual NMAC trajectories to collisions. When two aircraft are on course for an NMAC, the automated separation assurance system provides several layers of safety for detecting and resolving the problem. This section models the potential failures of the automated separation assurance system via reliability diagrams and dynamic event trees. 
Figure 21 shows the high level sequence of events. The events are categorized into four timeframes, as described in Section 1.2. The initiating event of the tree is a nonnormal event or blunder in which two aircraft are suddenly on course for an NMAC. Depending on the geometry of the scenario (see Figure 13) and the aircraft speeds, the time to the NMAC varies. Thus, the starting point of the dynamic event tree can be in any one of the four timeframes (strategic, tactical intent-based, tactical state-based, TCAS). The time counts down to zero and the final possible outcomes of the event tree are: (a) resolution of the conflict, (b) occurrence of an NMAC, or (c) a collision.

Two aircraft are on course for an NMAC

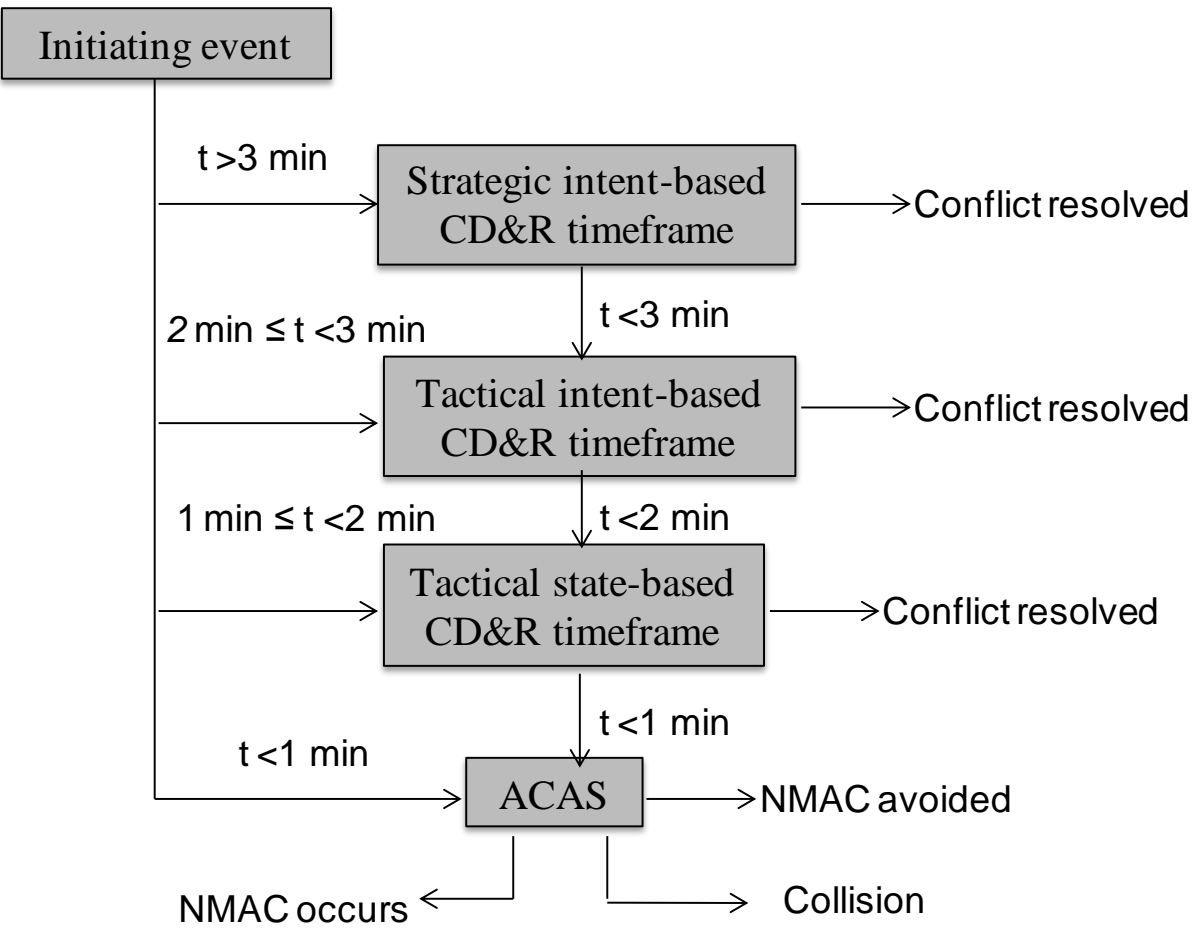

Figure 21 The overall flow of dynamic event tree 
Figure 22 shows the generic sub-tree for each timeframe. Each box represents a particular state at some time $t$ (the time left until the NMAC occurs). Arrows represent possible transitions. The event tree is said to be dynamic because the transition probability depends on both the state of the system as well as the time $t$. Loops indicate the possibility of repeating states over multiple time steps. $\Delta_{i}$ is the assumed time step in timeframe $i$, where $i=1,2,3$, 4. In later time frames (closer to the NMAC), smaller values for $\Delta_{i}$ are used. $T_{i}$ is the time threshold for timeframe $i$. If $t$ is equal or less than $T_{i+1}$, the flow goes to the next timeframe. 


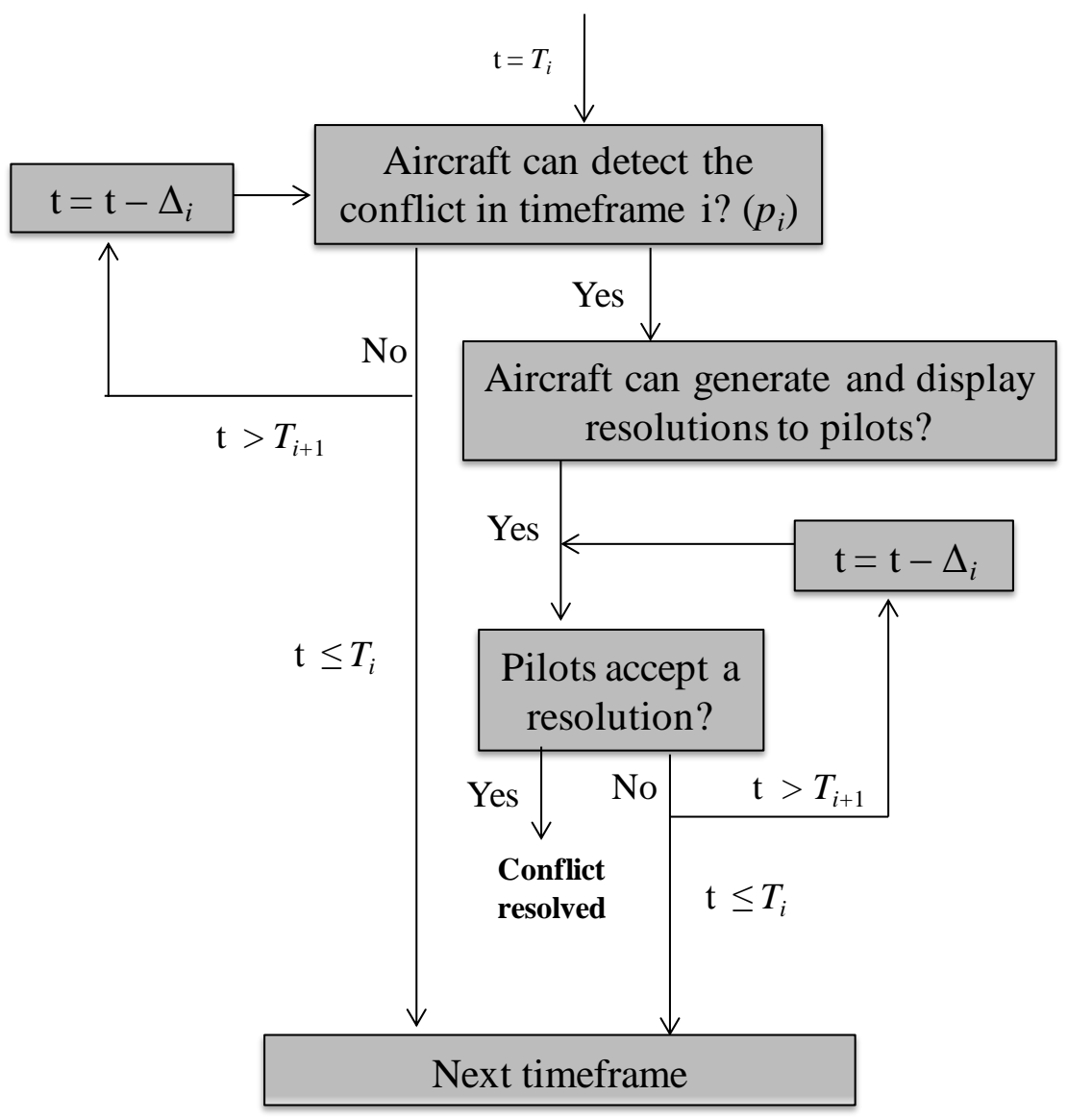

Figure 22 Generic sub-tree for each timeframe

Each sub-tree within a timeframe models the CD\&R function in terms of detection, resolution generation, display (visual and aural), and pilot response. In Figure 22 , the left loop shows the detection performance. The conflict-detection function operates over multiple time steps within the timeframe. To determine the detection probability, a model given by Erzberger et al. [1997] is used. The detection probability is a function of the time prior to the NMAC and the conflict angle. It is assumed that the cross-track position prediction error is normally distributed with constant root mean square in time. The along-track position prediction error is also normally distributed but 
with a root mean square that increases linearly in time. Figure 23 shows sample conflict detection probabilities in time for different conflict angles, based on our implementation of the model in Erzberger et al. [1997], the lane-change event corresponds to the solid line, the simultaneous lane-change event corresponds to the dashed-point line, and the lane change-overtaking event corresponds to the dashed line in which CPA refers to closest point of approach.

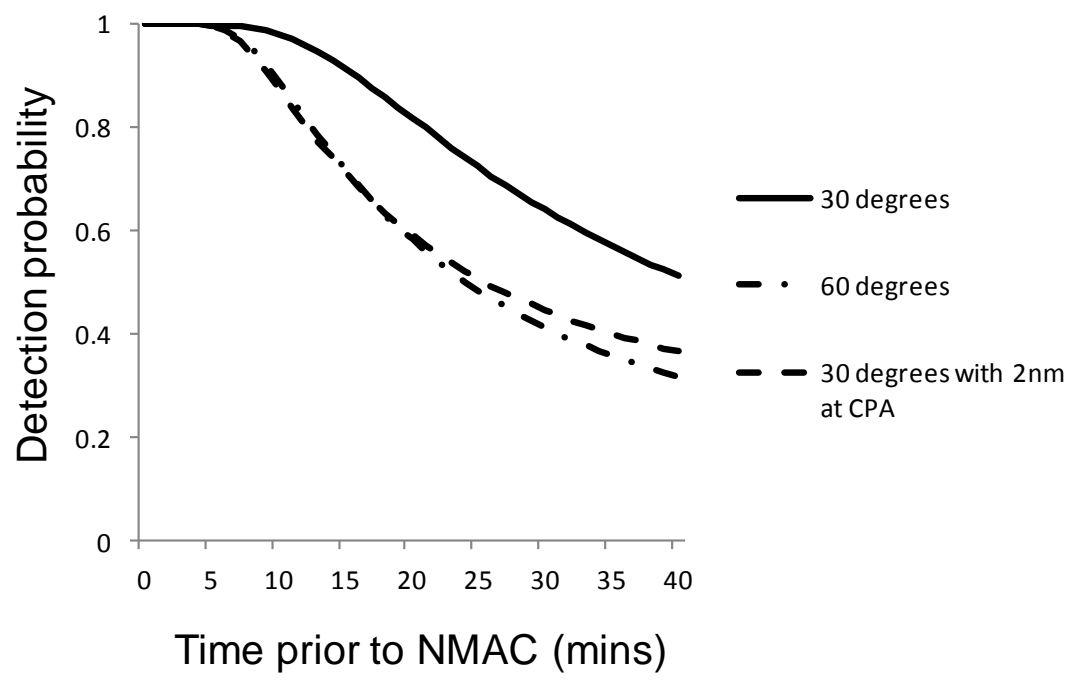

Figure 23 Conflict detection probability

The probabilities of successfully generating a resolution and displaying the resolution to the pilot are modeled via reliability diagrams. Figure 24 shows the reliability diagram of the strategic intent-based function. The output is whether aircraft 1 can generate a resolution and communicate the resolution to the pilot. For the strategic timeframe, it is assumed that only one aircraft, namely the following aircraft is responsible to execute a resolution to avoid the other aircraft. The resolution is provided 
by the strategic intent-based trajectory generator and is updated in the flight management system including time of arrival, fuel burn, etc. The resolution is displayed to the pilot via a navigation display.

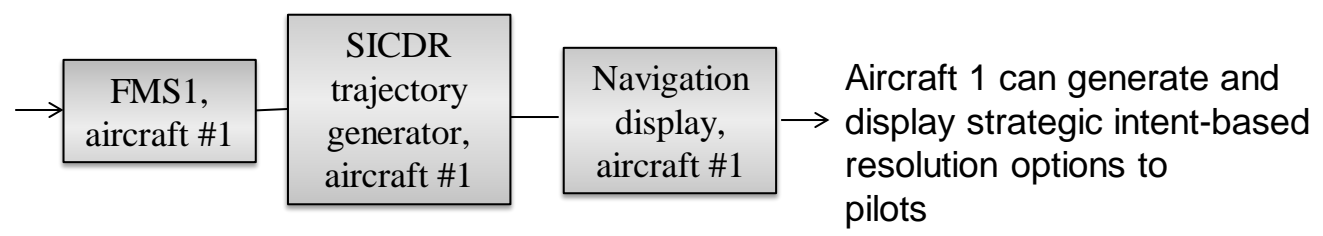

Figure 24 Reliability diagram of strategic intent-based function

Figure 25 shows the reliability diagram of the tactical intent-based function. The tactical intent-based function starts working when the NMAC is predicted to occur in 3 minutes or less. It first generates the planning trajectory prediction of both aircraft based on the position, velocity, flight plan information and flight management requirements for the aircraft. While flight plan information is used in detecting the conflicts, the maneuvers themselves, being tactical in nature, do not return the aircraft back to their original flight plans. The resolutions are communicated to the pilots via both aural and navigation display systems. Unlike the navigation display in the strategic intent-based function, the primary flight display does not show waypoints. It shows airspeed, altitude, and heading, etc. For a pair of aircraft in conflict, aircraft 1 and aircraft 2 , the conflict can be resolved as long as at least one aircraft executes the tactical intent-based resolution. 


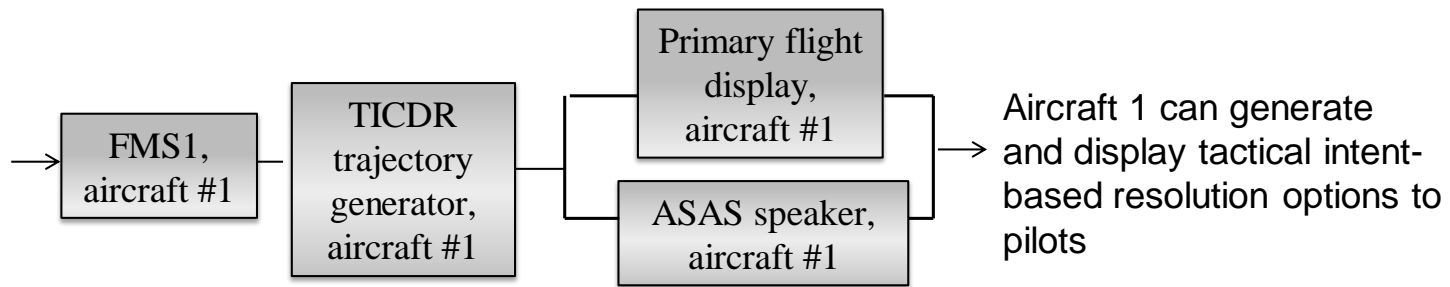

Figure 25 Reliability diagram of tactical intent-based function

The tactical state-based function starts working when the NMAC is predicted to occur in 2 minutes or less. It generates a state-based trajectory prediction (without intent information). The conflict resolution does not need to consider flight plan information or the FMS ([Wing and Cotton 2011], p.41). The primary goal is to resolve the conflict in a short amount of time.

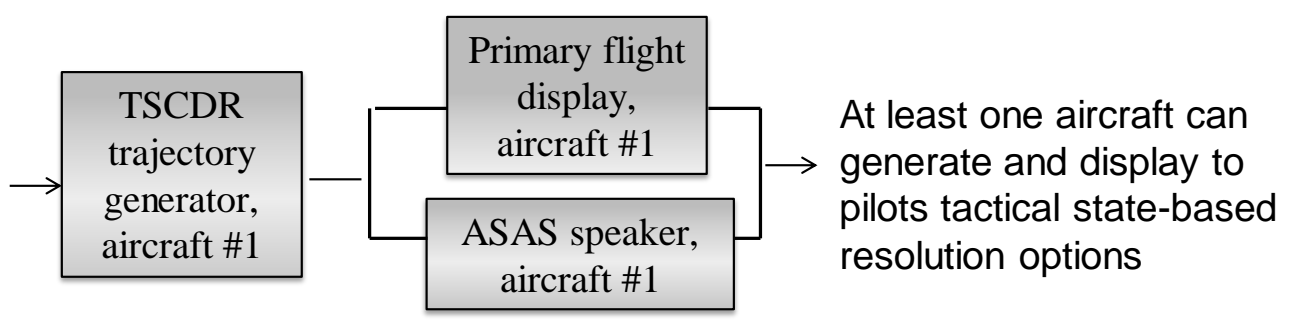

Figure 26 Reliability diagram of tactical state-based function

Figure 27 shows the reliability diagram for the TCAS function. The reliability diagram of TCAS has only one path meaning that all components must be working for the TCAS system to work. This assumes that both aircraft need to execute resolution maneuvers to avoid an NMAC. The components include the transponder, speaker and other components. A similar decomposition was made in Blum et al. [2010]. The failure of any component can cause the failure of TCAS. 


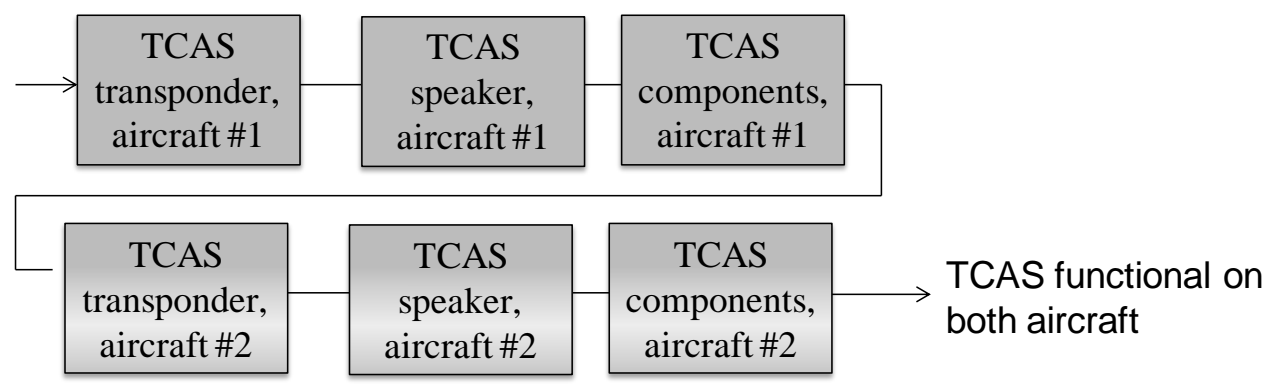

Figure 27 Reliability diagram of TCAS function

\subsection{Difference between Events Sequence Leading to Collisions}

Four dynamic event trees are created corresponding to the four different conflict scenarios in Figure 13. The main differences between the four trees are: 1) The initiating times prior to the NMAC are different, and 2) The conflict angles are different, so the resulting conflict-detection probabilities are different.

For example, the starting time of an overtaking scenario is usually more than 3 minutes prior to the NMAC (Figure 28). If the overtaking scenario occurs at 5 nautical miles separation and the relative velocity between the following aircraft and the leading aircraft is 20 knots, the time prior to the NMAC is 15 minutes at that point. For the lanechange scenario, the distance between the centerlines of two parallel lanes is 8 nautical miles. The speed of the aircraft is 350 knots. Aircraft 1 follows a 30 degree path to perform lane change maneuver. It takes the aircraft 2.7 minutes to have an NMAC with another aircraft in the adjacent lane. In this case, the dynamic event tree starts from tactical intent-based time frame. 


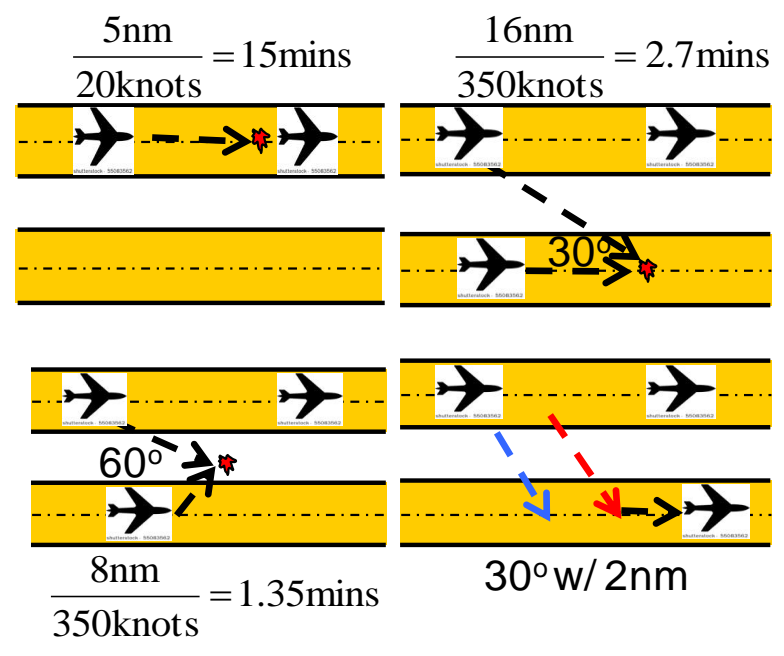

Figure 28 A simple calculation to estimate the starting point of lane change maneuver

Different types of collision scenarios determine the starting onboard conflict detection and resolution function in terms of the time prior to the NMAC (Table 7).

Table 7 Starting functions for collision scenarios

\begin{tabular}{|c|c|}
\hline Collision scenarios & $\begin{array}{c}\text { Starting onboard conflict detection and } \\
\text { resolution function }\end{array}$ \\
\hline Overtaking & Strategic intent-based function \\
\hline Lane change & Tactical state-based function \\
\hline Simultaneous lane change & Tactical state-based function \\
\hline Lane change and overtaking & $\begin{array}{c}\text { Strategic intent-based function or tactical } \\
\text { state-based function }\end{array}$ \\
\hline
\end{tabular}

The second difference between events is the conflict angle (Figure 29). The overtaking event has a 0 -degree conflict angle, the lane change event has a 30 -degree 
conflict angle, the simultaneous lane change event has a 60-degree conflict angle, the lane-change and overtaking event has 30-degree conflict angle. In the latter case, the separation is the Closest Point of Approach (CPA) when the aircraft reaches the center line of the destination lane. In this analysis, it is assumed that the lane-change and overtaking event uses the conflict detection probability in which the CPA is 2 nautical miles (The conflict detection probability is similar to those with other CPAs within 2 nautical miles.)

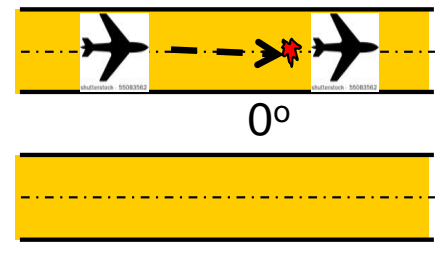

1. Overtaking

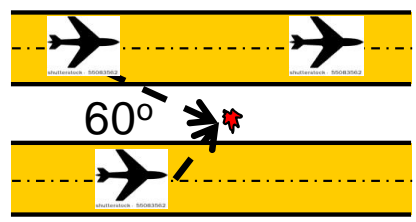

3. Simultaneous lane change

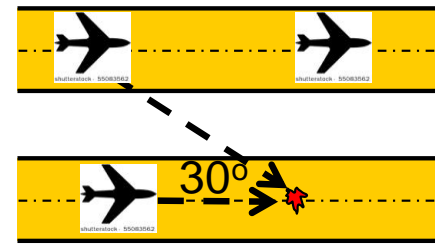

2. Lane change

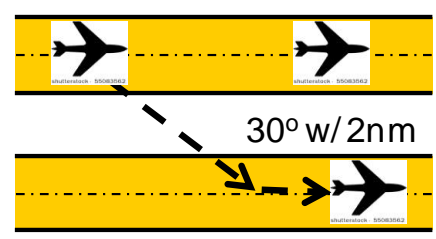

4. Lane change and overtaking

Figure 29 Conflict angles of the events

Erzberger et al. [1997] provide the conflict detection probability as a function of conflict angle as shown in Figure 29. In the dynamic event tree, the conflict detection 
probabilities are different between events for a given onboard conflict detection and resolution function.

\subsection{Sample Calculation}

We first consider a sample calculation in which the NMAC trajectory occurs as a result of a pilot blunder. In this case, Equation 3 can be written:

$$
\text { Pr \{collision }
$$

$$
=\sum_{i=1}^{4} \operatorname{Pr}\{\text { potental NMAC trajectory } i\}
$$

$* \operatorname{Pr}\{$ pilot blunder | potential NMAC trajectory $i\}$

$* \operatorname{Pr}\{$ collision | potential NMAC trajectory $i$, pilot blunder $\}$

The first term, $\operatorname{Pr}\{$ potential NMAC trajectory $i\}$, comes from the simulation. The second term, $\operatorname{Pr}\{$ pilot blunder | potential NMAC trajectory $i\}$, is a fixed value of $1.5 \mathrm{E}-5$ (Section 5.2.2). The calculation of the third term is illustrated here for the lane-change scenario $(i=2)$. The other scenarios are handled similarly.

For the lane-change scenario, the dynamic event tree starts from the tactical intent-based timeframe ( 3 minutes or less to NMAC), due to the proximity between the two lanes (Section 5.4). Now, evaluation of the dynamic event tree depends on the states of the functions in the reliability diagrams (Figure 24 - Figure 27). That is, failures of the reliability functions render certain $C D \& R$ functions non-operational. If the CD\&R function for a particular timeframe is non-operational, then that timeframe is "skipped" in 
the evaluation of the event tree. For example, if aircraft 1 cannot generate or display tactical intent-based resolution options to the pilots (Figure 25), then the CD\&R function is effectively non-operational, so the tactical intent-based timeframe is skipped in the event tree. Table 8 shows the set of relations between the reliability diagram functions and the event trees. The locatability function is critical (Figure 20), because the ability to locate other aircraft is necessary for the $C D \& R$ functions in the first three timeframes. (The TCAS timeframe uses a separate independent transponder interrogation to locate aircraft, so it is not affected by a loss of locatability.)

Table 8 Relationship between reliability diagrams and event trees, for one aircraft $(1=$ working, $0=$ failed $)$

\begin{tabular}{|c|c|c|c|c|}
\hline \multicolumn{2}{|c|}{ Reliability Diagrams } & \multicolumn{2}{c|}{ Event Tree Calculation } \\
\hline $\begin{array}{c}\text { Locatability } \\
\text { (Figure xx) }\end{array}$ & $\begin{array}{c}\text { Tactical } \\
\text { intent-based } \\
\text { (Figure xx) }\end{array}$ & $\begin{array}{c}\text { Tactical } \\
\text { state-based } \\
\text { (Figure xx) }\end{array}$ & $\begin{array}{c}\text { CD\&R } \\
\text { tactical } \\
\text { intent-based } \\
\text { phase }\end{array}$ & $\begin{array}{c}\text { CD\&R } \\
\text { tactical state- } \\
\text { based phase }\end{array}$ \\
\hline 1 & 1 & 1 & Evaluate & Evaluate \\
\hline 1 & 0 & 1 & Skip & Evaluate \\
\hline 1 & 1 & 0 & Evaluate & Skip \\
\hline 1 & 0 & 0 & Skip & Skip \\
\hline 0 & 1 & 1 & Skip & Skip \\
\hline 0 & 0 & 1 & Skip & Skip \\
\hline 0 & 1 & 0 & Skip & Skip \\
\hline 0 & 0 & 0 & Skip & Skip \\
\hline
\end{tabular}

Now, dynamic event trees are evaluated for both aircraft in a pair, since each aircraft can detect and resolve the conflict. Thus, the previous table must be generalized to consider function states for both aircraft. This is shown in Table 9. In the table, $S_{i j}$ represents a scenario in which the tactical intent-based CD\&R is operational on $i(=0,1,2)$ 
aircraft in a pair and the tactical state-based CD\&R is operational on $j(=0,1,2)$ aircraft in a pair. This yields 9 unique event-tree structures. These trees are obtained as modifications to the full event trees (Figure 21and Figure 22) by skipping appropriate timeframes. The full table contains $2^{6}=64$ rows, corresponding to all combination of function states.

Table 9 Example of combinations of function status (2 aircraft)

\begin{tabular}{|c|c|c|c|c|c|c|}
\hline \multicolumn{6}{|c|}{ Reliability Diagrams } & $\begin{array}{c}\text { Event } \\
\text { Tree } \\
\text { State }\end{array}$ \\
\hline $\begin{array}{c}\text { Locatabilit } \\
y \\
\text { (aircraft 1) }\end{array}$ & $\begin{array}{c}\text { Tactical } \\
\text { intent- } \\
\text { based } \\
\text { (aircraft } \\
1)\end{array}$ & $\begin{array}{c}\text { Tactical } \\
\text { state- } \\
\text { based } \\
\text { (aircraft } \\
1)\end{array}$ & $\begin{array}{c}\text { Locatabilit } \\
y \\
\text { (aircraft 2) }\end{array}$ & $\begin{array}{l}\text { Tactical } \\
\text { intent- } \\
\text { based } \\
\text { (aircraft } \\
\text { 2) }\end{array}$ & $\begin{array}{c}\text { Tactical } \\
\text { state- } \\
\text { based } \\
\text { (aircraft } \\
\text { 2) }\end{array}$ & $S_{i j}$ \\
\hline 1 & 1 & 1 & 1 & 1 & 1 & $S_{22}$ \\
\hline 0 & 1 & 1 & 1 & 1 & 1 & $S_{11}$ \\
\hline 1 & 1 & 0 & 0 & 1 & 0 & $S_{10}$ \\
\hline & $\ldots$ & & & $\ldots$ & $\cdots$ & \\
\hline
\end{tabular}

The final conditional collision probability is a calculated as a weighted sum of the 9 event trees:

Pr\{collision | potential NMAC trajectory 2, pilot blunder \}

$=\sum_{\mathrm{i}} \sum_{j} \operatorname{Pr}\left\{\right.$ state $\mathrm{S}_{\mathrm{ij}} \mid$ potential NMAC trajectory 2, Pilot blunder $\}$

$* \operatorname{Pr}\left\{\right.$ collision | potential NMAC trajectory 2 , pilot blunder , state $\left.S_{\mathrm{ij}}\right\}$ 
The next example considers a scenario in which the NMAC trajectory occurs as a result of a loss of locatability. This case is somewhat simpler. If the aircraft loses the ability of locate other aircraft, all CD\&R functions except TCAS fail to work. The baseline dynamic event tree is reduced to a simple tree with only TCAS. In this case, Equation 3 becomes:

$$
\begin{aligned}
\operatorname{Pr}\{\text { collision }\} & =\sum_{i=1}^{4} \operatorname{Pr}\{\text { potential NMAC trajectory } i\} * \operatorname{Pr}\{\text { loss of locatability }\} \\
& * \operatorname{Pr}\{\text { collision } \mid \text { TCAS }\}
\end{aligned}
$$

The dynamic event tree is used to calculate the conditional collision probability $\operatorname{Pr}\{$ collision | TCAS|. This probability can be decomposed as follows:

$$
\operatorname{Pr}\{\text { collision } \mid \text { TCAS }\}=\operatorname{Pr}\{\text { loss of TCAS }\} * p_{f}+(1-\operatorname{Pr}\{\text { loss of TCAS }\}) * p_{w}
$$

where $p_{f}$ is the probability of a collision if TCAS fails and $p_{w}$ is the probability of a collision if TCAS works. If TCAS fails, then an NMAC is assumed to occur with certainty, and the NMAC may also result in a collision by chance with probability $p_{f}$. If TCAS works, the NMAC can still occur if the pilot does not execute the resolution. The failure probability of TCAS is

$$
\operatorname{Pr}\{\text { loss of TCAS }\}=1-\sum_{i=13}^{18}\left(1-\mathrm{p}_{\mathrm{i}}\right)
$$


where components 13 thru 18 are the transponder, the speaker and other sub-components of TCAS (see Appendix for the assumed numerical values).

Table 10 shows part of the transition matrix that is built from the dynamic event tree. It includes start state-time pairs, end state-time pairs and their transition probabilities. The left two columns are the start state-time pairs. The start state-time pair is the current state at a given time step. The third and fourth columns are the end state-time pairs. The end state-time pair is the state that moves from the start state-time pair. The other columns are the transition probability from the start state-time pair to the end state-time pair for different status of conflict detection and resolution functions. The whole transition matrix contains 9 columns of transition probabilities corresponding to 9 states of conflict detection and resolution functions. One start state-time pair can move to several end state-time pairs. For example, if the start state-time pair is tactical intentbased CD at 180 seconds prior to the NMAC, the end state-time pairs include: 1) Tactical intent-based CD override. This means that the tactical intent-based starts working. 2) Tactical state-based CD at 120 seconds prior to the NMAC. This means that tactical intent-based function fails to detect the conflict. 3) TCAS. Both tactical intent-based function and tactical state-based function fail. One end state-time pair can also have several start state-time pairs. By analyzing the relation between state-time pairs, the objective is to calculate the probability of the collision state at time 0 for all states of conflict detection and resolution functions. 
Table 10 Example of dynamic event tree calculation

\begin{tabular}{|c|c|c|c|c|c|}
\hline Start time & Start state & End time & End state & $\mathrm{S}_{22}$ & $\mathrm{~S}_{21}$ \\
\hline 180 & tactical intent-based CD & 180 & tactical intent-based CD override & 1 & 1 \\
\hline 180 & tactical intent-based CD & 120 & tactical state-based CD & $1.00 \mathrm{E}-06$ & $1.00 \mathrm{E}-06$ \\
\hline 180 & tactical intent-based CD & 60 & TCAS & 0 & 0 \\
\hline 180 & tactical intent-based CD override & 160 & Resolved & 0.82307 & 0.82307 \\
\hline 180 & tactical intent-based CD override & 160 & tactical intent-based CD override & 0.17693 & 0.17693 \\
\hline 160 & tactical intent-based CD override & 140 & Resolved & 0.84144 & 0.84144 \\
\hline 160 & tactical intent-based CD override & 140 & tactical intent-based CD override & 0.15856 & 0.15856 \\
\hline 140 & tactical intent-based CD override & 120 & Resolved & 0.85932 & 0.85932 \\
\hline 140 & tactical intent-based CD override & 120 & tactical state-based CD & 0.14068 & 0.14068 \\
\hline 120 & tactical state-based CD & 120 & tactical state-based CD override & 1 & 1 \\
\hline 120 & tactical state-based CD & 60 & TCAS & $1.00 \mathrm{E}-06$ & $1.00 \mathrm{E}-06$ \\
\hline 120 & tactical state-based CD override & 110 & Resolved & 0.83088 & 0.63913 \\
\hline 120 & tactical state-based CD override & 110 & tactical state-based CD override & 0.16912 & 0.36087 \\
\hline
\end{tabular}

The overall collision probability is calculated by summing up all the conditional collision probabilities given the status of conflict detection and resolution functions. 


\section{CHAPTER 6 RESULTS FOR SIMULATION AND DYNAMIC EVENT TREE ANALYSIS}

This chapter gives results for the flow corridor simulation and the dynamic event tree analysis. Section 6.1 discusses the relation between the occurrence rates of potential events and the design parameters. A full factorial analysis is conducted to identify critical parameters that affect the rates. Section 6.2 discusses the calculation of dynamic event trees to estimate the conditional collision probability given the occurrence of events. In terms of the status of the onboard conflict detection and resolution functions, the conditional collision probability is calculated for each combination of function states. Two case studies discussed in Chapter 5 are used to illustrate the process of calculating the overall collision probabilities.

\subsection{Flow Corridor Simulation Results}

This section gives numerical results for the corridor simulation. Five parameters are varied to determine the impact on potential events: 1) minimum separation, 2) fleet mix, 3) initial separation, 4) target speed, and 5) relative speed threshold. Table 5 shows the baseline values and ranges. The experiments vary one design parameter at a time using baseline value for the other parameters. A full-factorial experiment is given later. The experiments assume that only two types of aircraft are in the flow corridor (B737 and B767). 
Table 11 Design of experiments

\begin{tabular}{|c|c|c|}
\hline Design parameters & Baseline value & Value range \\
\hline Minimum separation & $5 \mathrm{~nm}$ & $0.5-6 \mathrm{~nm}$ \\
\hline Fleet mix & $20 \%$ B $737,80 \%$ B767 & $\begin{array}{l}0-100 \% \text { of } B 737 \\
0-100 \% \text { of } B 767\end{array}$ \\
\hline $\begin{array}{c}\text { Initial separation index } \\
\text { (average initial separation }= \\
\text { minimum separation } \times \\
\text { initial separation index) }\end{array}$ & 1.3 & $1.3-2.5$ \\
\hline $\begin{array}{c}\text { Target velocity standard } \\
\text { deviation }\end{array}$ & 5 knots & $2-5 \mathrm{knots}$ \\
\hline Relative speed threshold & $10 \mathrm{knots}$ & 5-30knots \\
\hline
\end{tabular}

Figure 30 shows the impact of minimum separation. As the minimum separation decreases, the potential-event rates increase non-linearly. The overtaking event has the highest occurrence rate. This is because the overtaking event is, by definition, a required condition for the other events. The lane change scenario has a lower occurrence rate than the lane-change-and-overtaking scenario because the former requires an NMAC with the adjacent aircraft, while the latter requires a less restrictive loss of separation followed by an overtaking speed. The simultaneous lane change scenario has the lowest rate because it requires two potential lane change events at roughly the same time and location. 


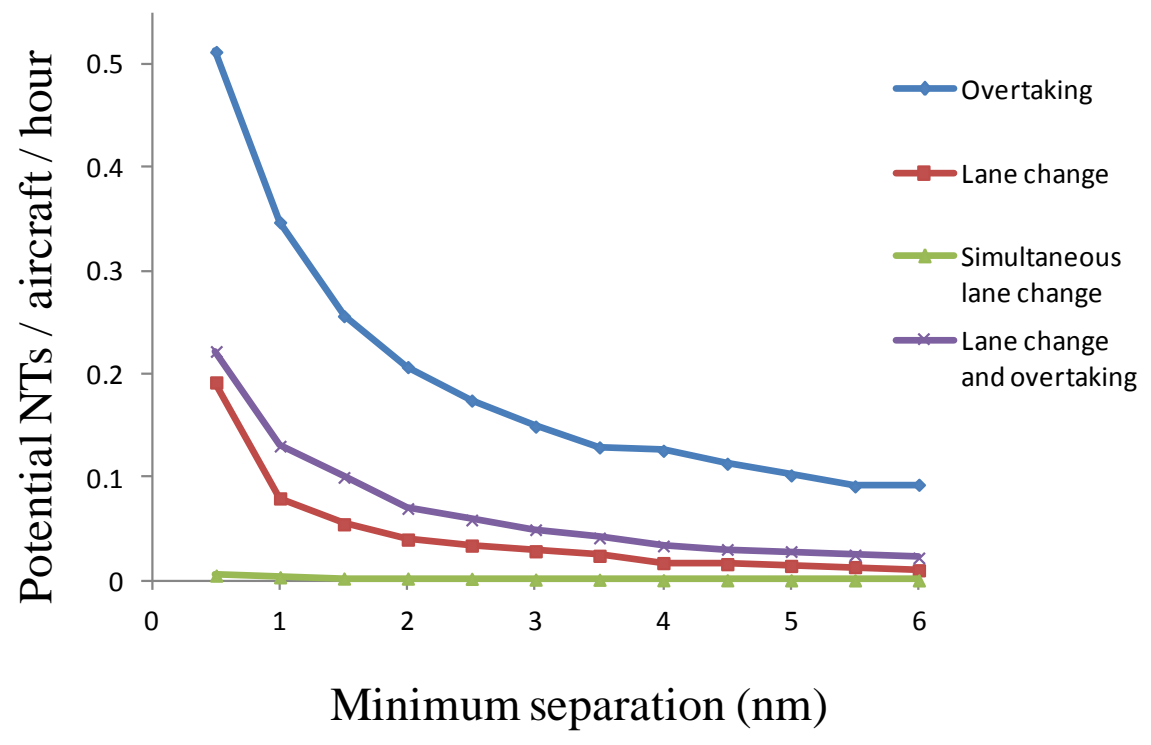

Figure 30 Experiment of varying minimum separation

Figure 31 shows the impact of fleet mix. The rates of potential NMAC trajectories are near zero at the boundaries, because all flights have the same average target speed. The maximum occurs with a fleet mix of roughly 30 percent slower aircraft. Intuitively, the slower aircraft have a higher relative impact in terms of increasing the interactions between slow and fast aircraft. 


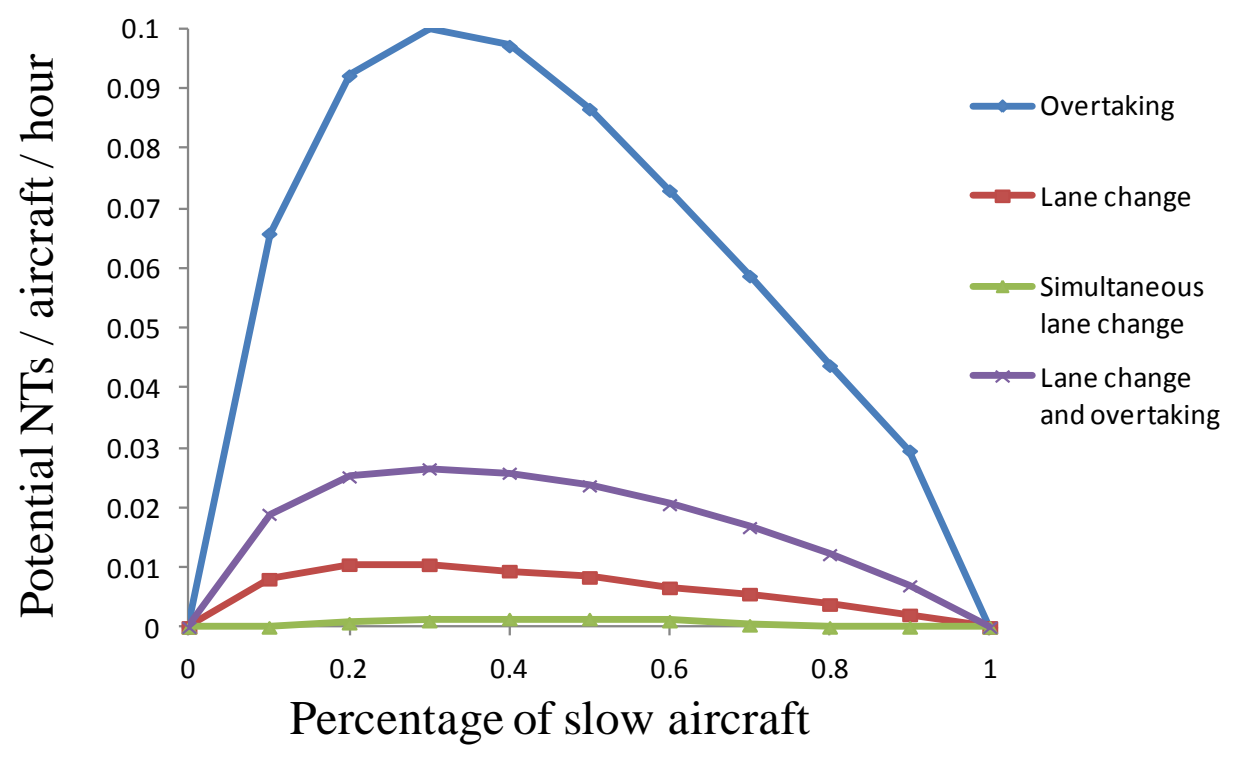

Figure 31 Experiment of varying fleet mix

Figure 32 shows the impact of the average initial separation. The initial separation equals the minimum separation multiplied by the initial separation index plus an exponentially distributed random variable. The occurrence rates increase when the standard deviation increases, because there is more variability in relative speed, resulting in more interactions. However, the overall effect is less than observed for some of the other parameters. 


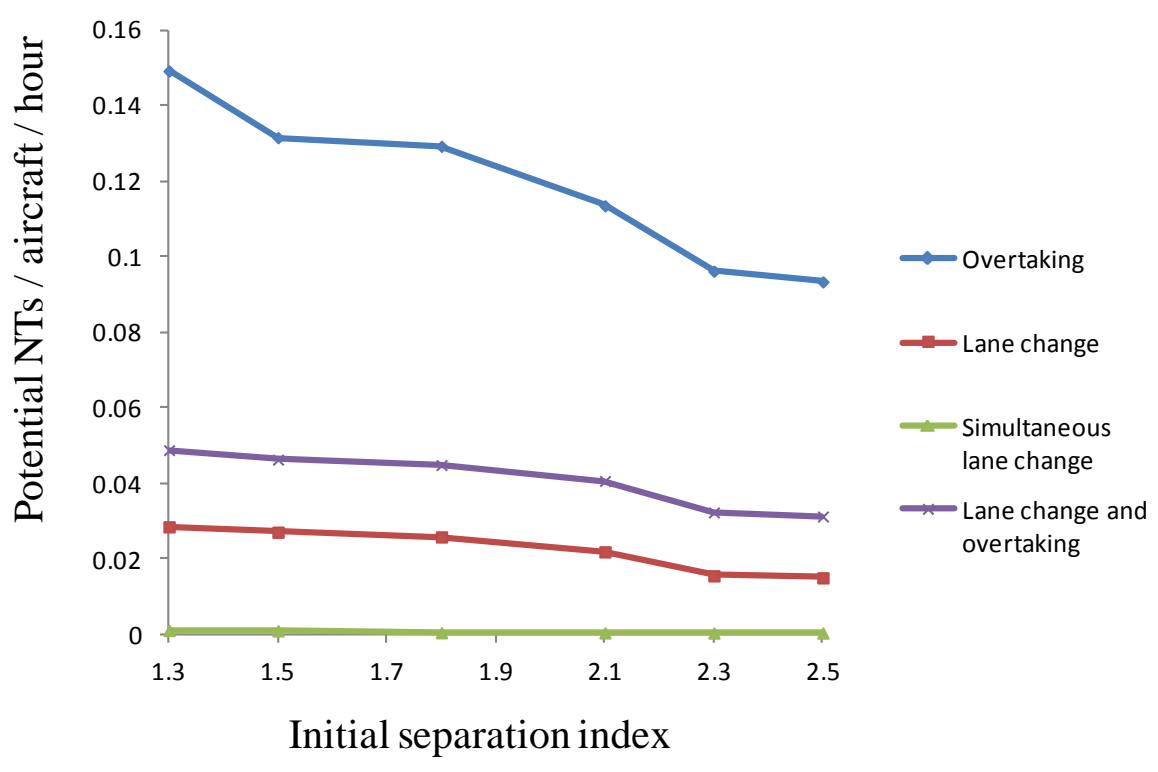

Figure 32 Experiment of varying initial separation

Figure 33 shows the impact of the standard deviation of the target speed. The figure shows that the occurrence rates increase when the standard deviation of the target velocity increases. This is because of the increased relative speed that results in more lane-change maneuvers. However, the overall rate of change is smaller than in previous figures because the mean of target velocities are unchanged. 


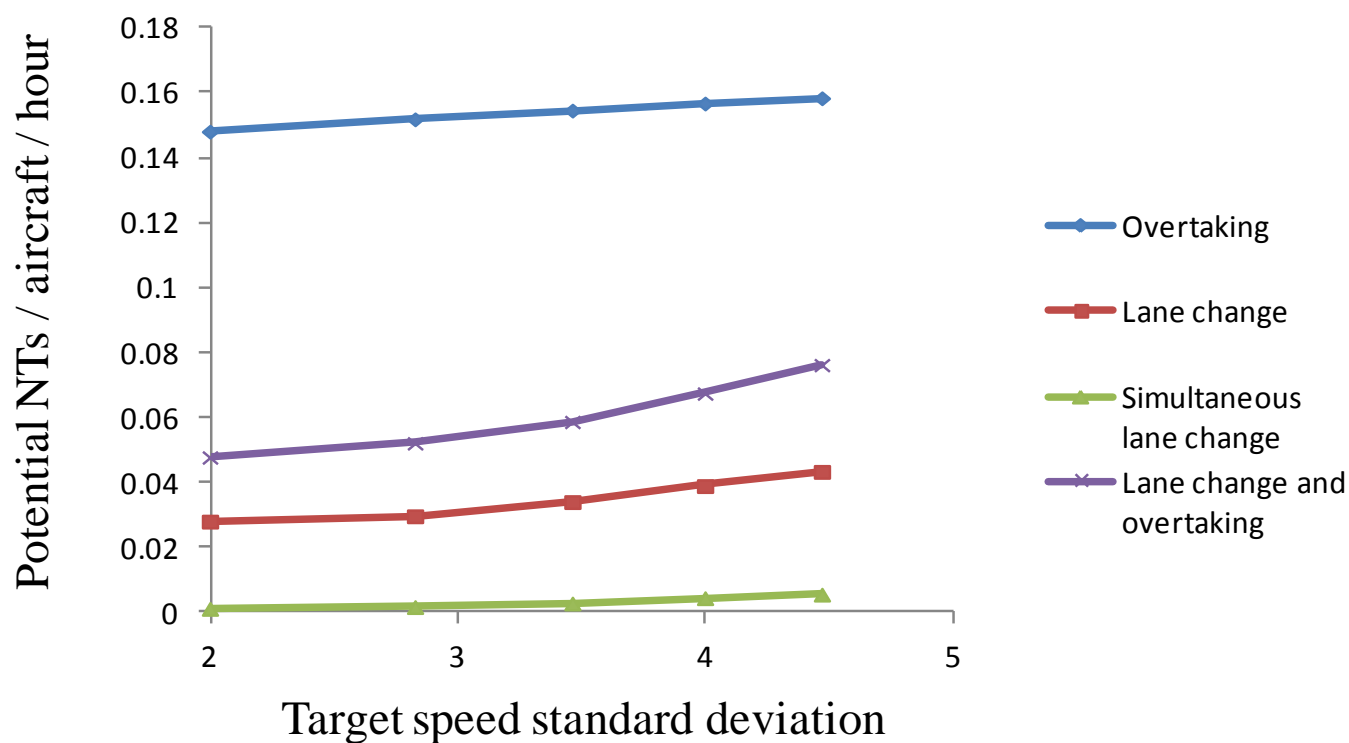

Figure 33 Experiment of varying standard deviation of target speed

Figure 34 shows the impact of the relative speed requirement. When the requirement increases, a higher fraction of faster aircraft behind slower aircraft reduce their speed rather than change lanes. If the relative speed requirement is high enough, virtually all of the aircraft reduce speed rather than change lanes. 


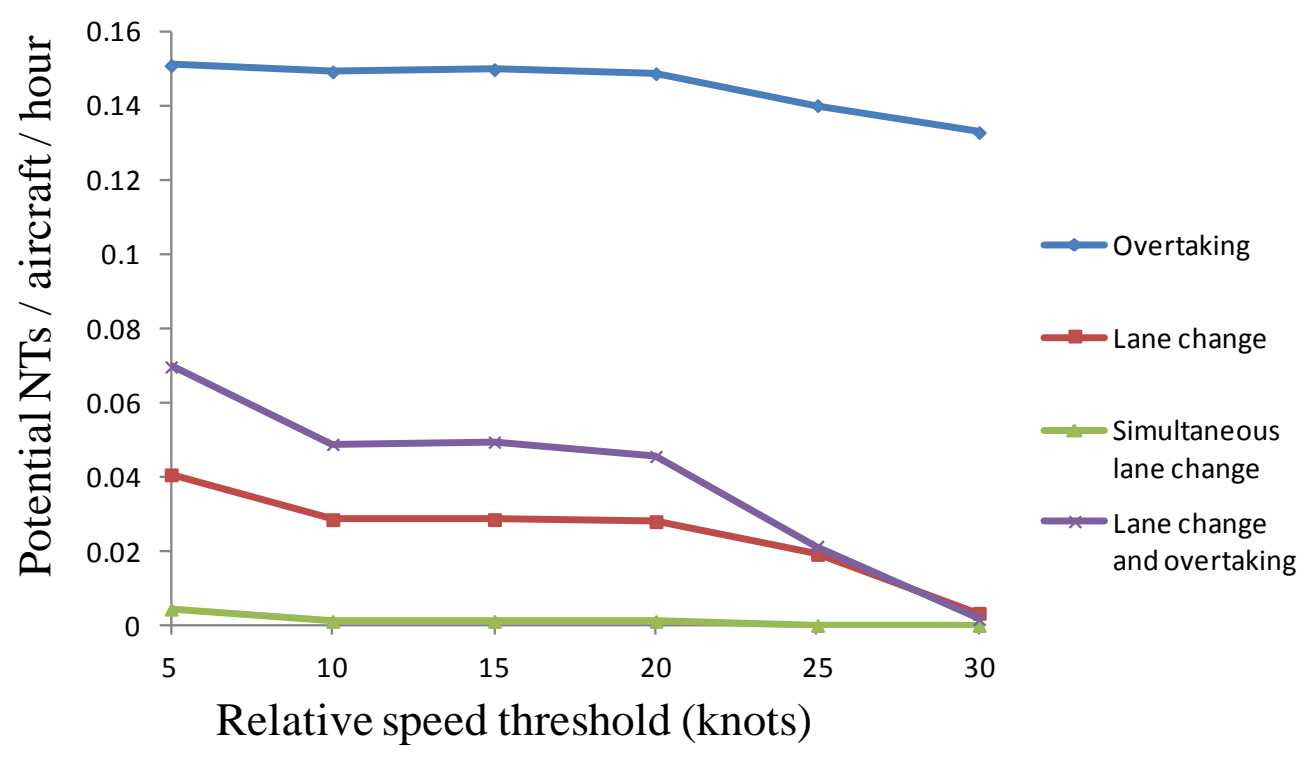

Figure 34 Experiment of varying relative speed threshold

The next experiment gives results using a more realistic fleet mix (rather than assuming two aircraft types). Table 12 shows the top seven aircraft types from one month of landing track data at ORD (2009 data). The percentages are normalized to sum to $100 \%$.

Table 12 Simulation Baseline

\begin{tabular}{|c|c|c|}
\hline Aircraft type & Percentage & $\begin{array}{c}\text { Maximum Mach } \\
\text { Number }\end{array}$ \\
\hline EMB145 & $40 \%$ & 0.78 \\
\hline B757 & $16 \%$ & 0.86 \\
\hline A320 & $11 \%$ & 0.82 \\
\hline B737 & $10 \%$ & 0.82 \\
\hline B767 & $9 \%$ & 0.86 \\
\hline DC10 & $8 \%$ & 0.88 \\
\hline A319 & $6 \%$ & 0.82 \\
\hline
\end{tabular}


Figure 35 shows the effect of decreasing the minimum separation from 6 nautical miles to 1 nautical mile. The figure is analogous to Figure 30, except that it uses 7 aircraft types instead of 2 aircraft types. The occurrence rates have the same shape. However, the values in this figure are less than those in Figure 30 because the speed differences in the two-aircraft case are more extreme (slow / fast aircraft) than in the seven-aircraft case.

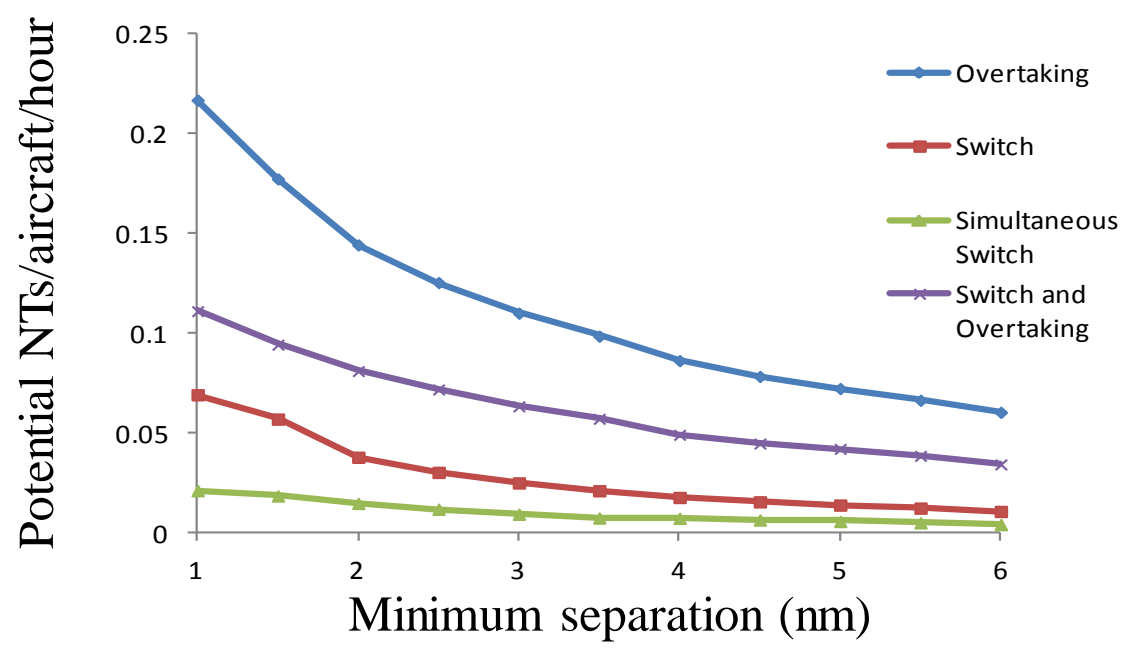

Figure 35 Baseline experiment

A limitation of the approach discussed above is that it does not consider interaction effects. In order for the results to be valid over a range of system parameter values, it must be assumed that the sensitivity of the system to one parameter does not depend on the values of the other parameters over this range (in other words, the response is additive to each parameter). A better way to conduct sensitivity analysis is to vary all parameters in a full factorial experiment. 
The procedure can be summarized as follows:

- Choose a low value (-) and high value (+) for each parameter

- Enumerate all $2^{n}$ parameter combinations, where $n$ is the number of parameters (each parameter can take its low and high value),

- Calculate the system response for each of the $2^{n}$ parameter combinations,

- Calculate the factor effects (described below).

In this case, a 5 factor- 2 level full factorial analysis is conducted to identify how the combination of design parameters affects the occurrence of potential NMAC trajectories. The higher value is represented as a plus sign in Figure 36 and the low value is represented as a minus sign. In total, there are 32 combinations. The last column represents the occurrence rates of potential NMAC trajectories. 


\begin{tabular}{|c|c|c|c|c|c|c|c|c|}
\hline \multirow[b]{2}{*}{ Value } & \multirow[b]{2}{*}{ RUN } & \multirow[b]{2}{*}{ Comb. } & \multicolumn{6}{|c|}{ Factors } \\
\hline & & & Fleet Mix & $\begin{array}{c}\text { Target } \\
\text { Speed } \\
\text { Standard } \\
\text { Deviation }\end{array}$ & $\begin{array}{c}\text { Initial } \\
\text { Separation }\end{array}$ & $\begin{array}{c}\text { Relative } \\
\text { Speed } \\
\text { Threshold }\end{array}$ & $\begin{array}{l}\text { Minimum } \\
\text { Separation }\end{array}$ & $\begin{array}{c}\text { Occurrence } \\
\text { Rates of } \\
\text { Events }\end{array}$ \\
\hline Hign (+) & & & $(0.2,0.8)$ & 5 & minsep*2.0 & 15 & 5 & \\
\hline \multirow[t]{33}{*}{ Low (-) } & & & $(0.8,0.2)$ & 2 & minsep* 1.3 & 5 & 3 & \\
\hline & 1 & -1 & - & - & - & - & - & $x_{1}$ \\
\hline & 2 & $a$ & + & - & - & - & - & $x_{2}$ \\
\hline & 3 & $b$ & - & + & - & - & - & $x_{3}$ \\
\hline & 4 & $a b$ & + & + & - & - & - & $\mathbf{x}_{4}$ \\
\hline & 5 & $\mathrm{C}$ & - & - & + & - & - & $\mathbf{x}_{5}$ \\
\hline & 6 & $\mathrm{ac}$ & + & - & + & - & - & $x_{6}$ \\
\hline & 7 & $\mathrm{bc}$ & - & + & + & - & - & $x_{7}$ \\
\hline & 8 & $a b c$ & + & + & + & - & - & $x_{8}$ \\
\hline & 9 & $\mathrm{~d}$ & - & - & - & + & - & $X_{9}$ \\
\hline & 10 & ad & + & - & - & + & - & $x_{10}$ \\
\hline & 11 & $b d$ & - & + & - & + & - & $x_{11}$ \\
\hline & 12 & abd & + & + & - & + & - & $x_{12}$ \\
\hline & 13 & $\mathrm{~cd}$ & - & - & + & + & - & $x_{13}$ \\
\hline & 14 & acd & + & - & + & + & - & $x_{14}$ \\
\hline & 15 & bcd & - & + & + & + & - & $x_{15}$ \\
\hline & 16 & abcd & + & + & + & + & - & $x_{16}$ \\
\hline & 17 & $\mathrm{e}$ & - & - & - & - & + & $x_{17}$ \\
\hline & 18 & ae & + & - & - & - & + & $x_{18}$ \\
\hline & 19 & be & - & + & - & - & + & $x_{19}$ \\
\hline & 20 & abe & + & + & - & - & + & $x_{20}$ \\
\hline & 21 & ce & - & - & + & - & + & $x_{21}$ \\
\hline & 22 & ace & + & - & + & - & + & $x_{22}$ \\
\hline & 23 & bce & - & + & + & - & + & $x_{23}$ \\
\hline & 24 & abce & + & + & + & - & + & $x_{24}$ \\
\hline & 25 & de & - & - & - & + & + & $x_{25}$ \\
\hline & 26 & ade & + & - & - & + & + & $x_{26}$ \\
\hline & 27 & bde & - & + & - & + & + & $x_{27}$ \\
\hline & 28 & abde & + & + & - & + & + & $X_{28}$ \\
\hline & 29 & cde & - & - & + & + & + & $x_{29}$ \\
\hline & 30 & acde & + & - & + & + & + & $x_{30}$ \\
\hline & 31 & bcde & - & + & + & + & + & $x_{31}$ \\
\hline & $32=2^{5}$ & abcde & + & + & + & + & + & $x_{32}$ \\
\hline
\end{tabular}

Figure 36 Full factorial analysis

The main effect of a parameter is the average value corresponding to the high values of the parameter minus the average value corresponding to the low values of the parameter. For example, the main effect of fleet mix is:

Fleet mix effect: $\frac{x_{2}+x_{4}+\ldots+x_{32}}{16}-\frac{x_{1}+x_{3}+\ldots+x_{31}}{16}$. 
The main effects are similar to varying one parameter at a time. However, because the main effect is obtained over a larger range of the parameter space, the estimate is more representative of the effects due to the parameter.

The interaction effect between two parameters measures the non-additive response of the system to the parameters. For example, a positive interaction means that increasing both parameters results in a collision probability value that is greater than the sum of the increases that would be obtained by varying each parameter individually.

The following example illustrates the calculation of the interaction effect for the two parameters, minimum separation and relative speed threshold. When the minimum separation is high (+), the average effect of the relative speed threshold (that is, the difference between the relative speed threshold-high value and the relative speed threshold-low value, holding the other parameters fixed is $\frac{\sum_{i=25}^{i=32} x_{i}-\sum_{i=17}^{i=24} x_{i}}{16}$. When the minimum separation is low (-), the average effect of the relative speed threshold is $\frac{\sum_{i=9}^{i=16} x_{i}-\sum_{i=1}^{i=8} x_{i}}{16}$. The interaction effect is the difference between the relative speed threshold effect when the minimum separation value is high minus the relative speed threshold effect when the minimum separation value is low, namely:

$$
\frac{\sum_{i=9}^{i=16} x_{i}+\sum_{i=25}^{i=32} x_{i}}{16}-\frac{\sum_{i=1}^{i=8} x_{i}+\sum_{i=17}^{i=24} x_{i}}{16}
$$

Figure 37 - Figure 41 shows the results of the full factorial analysis. Figure 37 is for the occurrence rates of potential NMAC trajectories of all collision scenarios. Figure 38 to Figure 41 correspond to the occurrence rates of potential NMAC trajectories of individual collision scenarios. The $x$-axis shows the main effects and interaction effects 
of the parameters on the occurrence rates of potential NMAC trajectories. A positive value means that increasing the parameter increases the occurrence rate; a negative value means that increasing the parameter decreases the occurrence rate. Longer bars correspond to higher impacts. The minimum-separation parameter has the largest impact on the occurrence rate of potential NMAC trajectories. This might be expected because the occurrence rate increases non-linearly when the minimum separation is reduced. The fleet-mix parameter has the largest impact on the occurrence rate of potential NMAC trajectories for lane change scenario. The fleet-mix parameter affects the number of lane change procedures and has higher impact on the lane change event than the overtaking scenario The relative speed threshold is the most critical design parameter for the occurrence rate of potential NMAC trajectories of the simultaneous lane change scenario. Since the target speed of each aircraft is a random variable, the relative speed threshold also affects the number of lane change procedures. It has a higher impact on the simultaneous lane change scenario than the lane change scenario because the simultaneous lane change scenario requires two pairs of aircraft to perform lane change procedures. The interaction effects between two parameters have relatively minor impacts on occurrence rates of potential NMAC trajectories compared to the main effects of parameters 


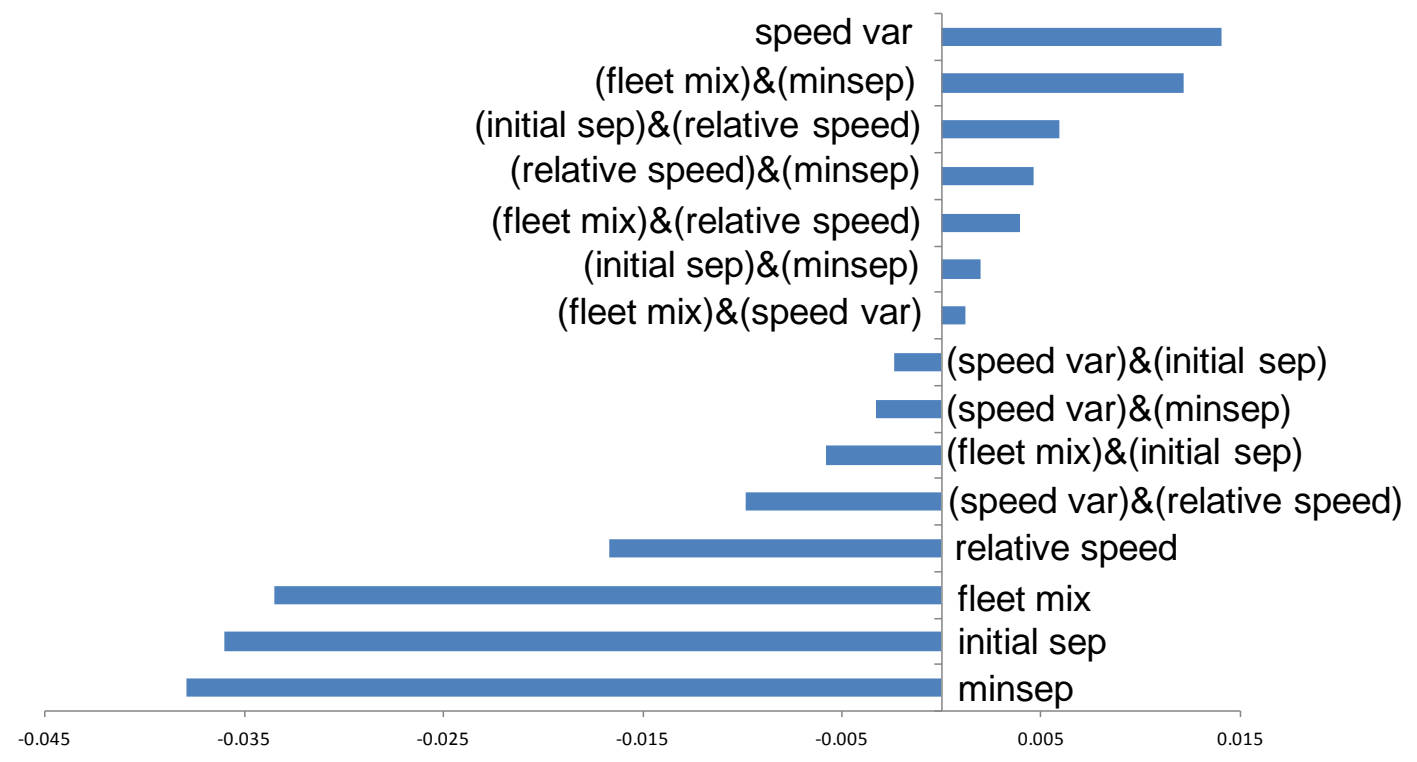

Figure 37 Results of full factorial analysis for collision scenarios (increasing parameter effect on potential NMAC trajectory rate)

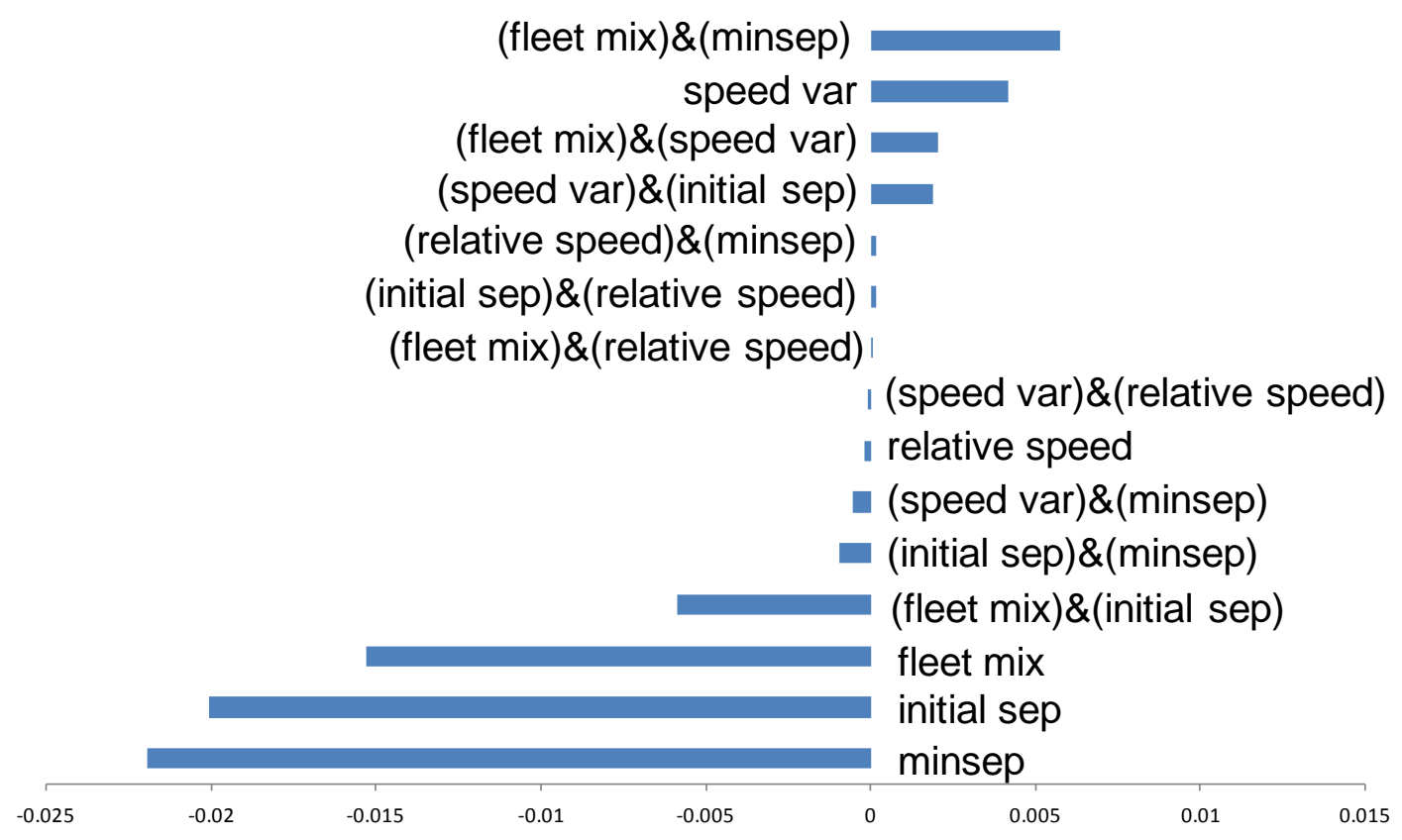

Figure 38 Results of full factorial analysis for overtaking scenario (increasing parameter effect on potential NMAC trajectory rate) 


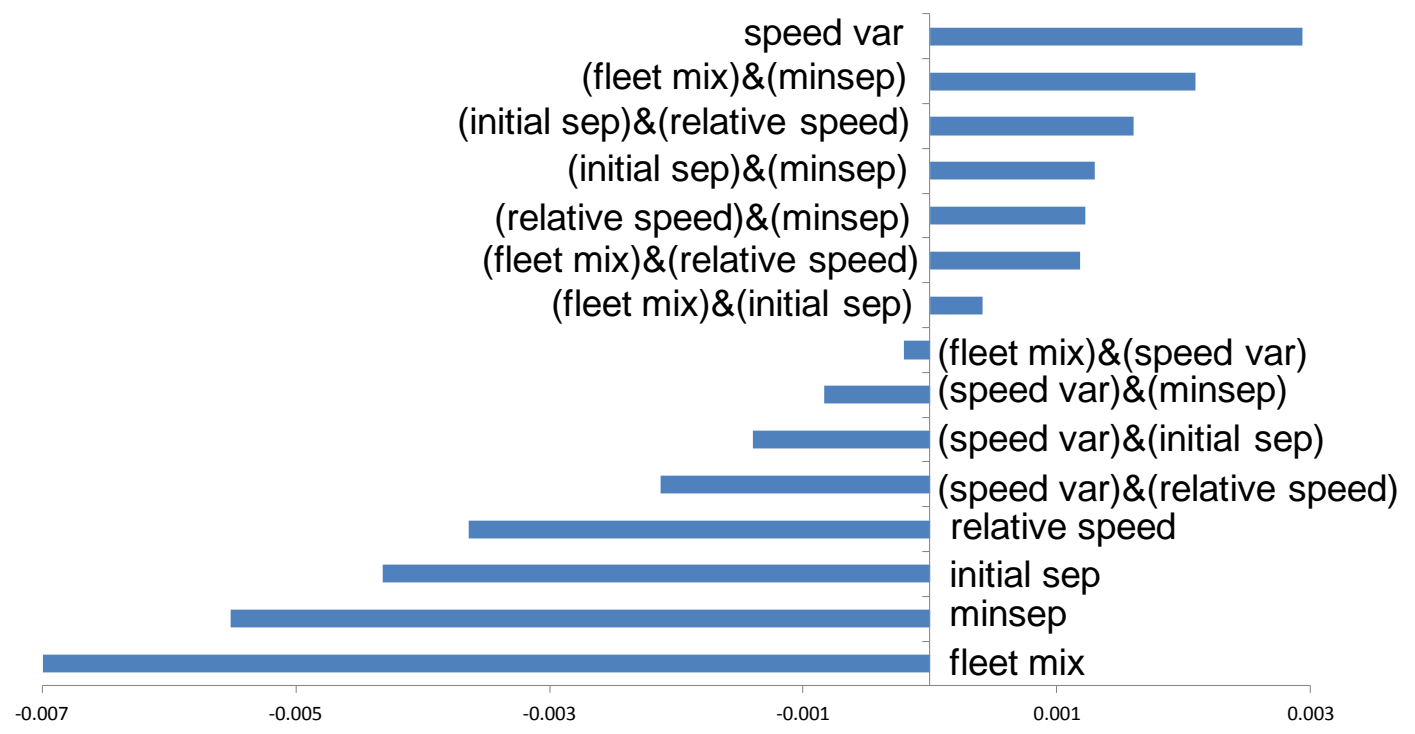

Figure 39 Results of full factorial analysis for lane change scenario (increasing parameter effect on potential NMAC trajectory rate)

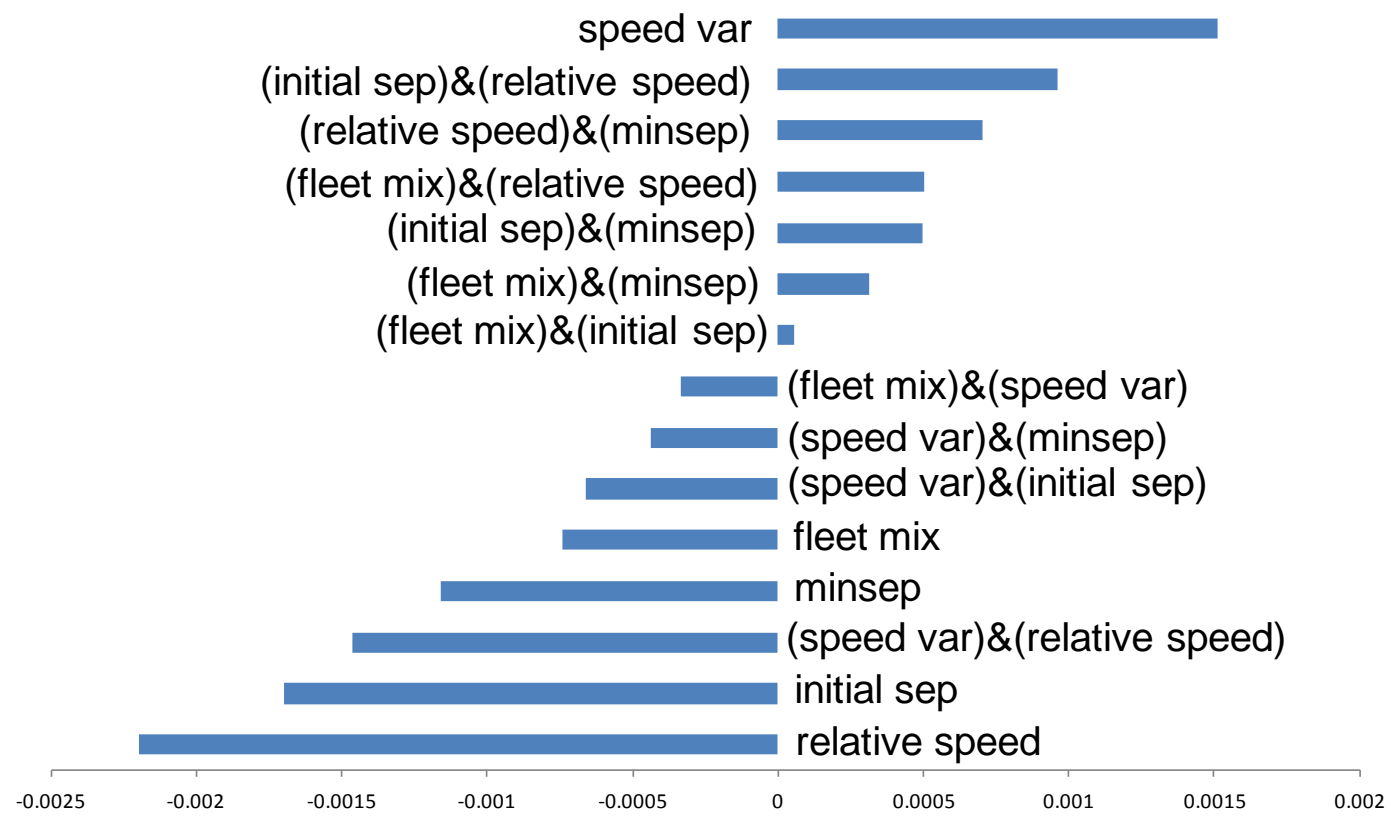

Figure 40 Results of full factorial analysis for simultaneous lane change scenario (increasing parameter effect on potential NMAC trajectory rate) 


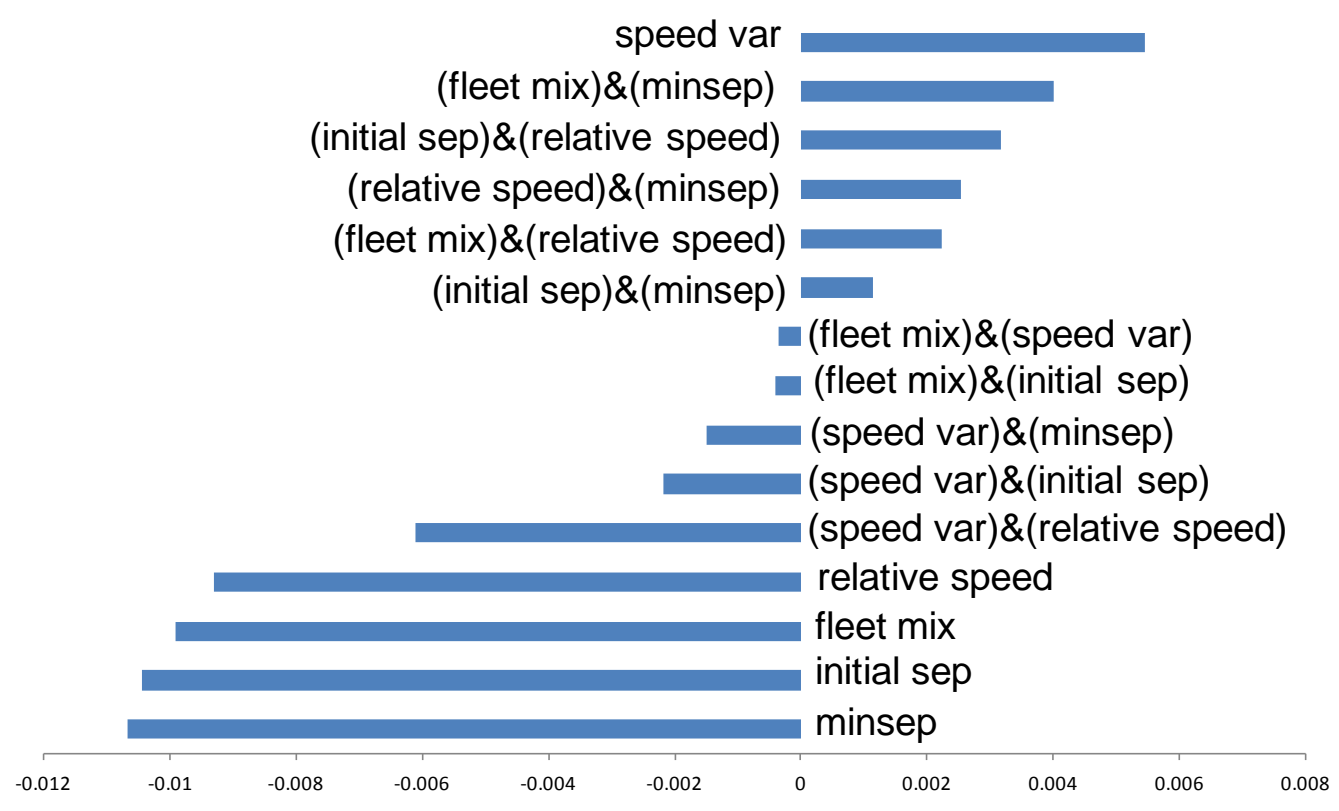

Figure 41 Results of full factorial analysis for simultaneous and lane change scenario (increasing parameter effect on potential NMAC trajectory rate)

\subsection{Results of Dynamic Event Tree Analysis}

This section discusses the conditional collision probability of the two case studies using dynamic event tree analysis discussed in Chapter 5. The conditional collision probability is the third part in Equation 3. The following paragraphs discuss the conditional collision probability of the two case studies.

The first case study involves a loss of locatability. A loss of locatability occurs when neither aircraft can locate the other. If neither aircraft can locate the other, then all onboard conflict detection and resolution functions fail and only TCAS is available to prevent the collision. The dynamic event trees are the same for each of the four collision scenarios (overtaking, lane change, etc.). The conditional collision probability is then calculated to be $2.54 \mathrm{E}-02$. 
The second case study involves pilot blunders. In the case of a pilot blunder, the dynamic event trees are used to model the subsequent $C D \& R$ functions that correct the blunder. The sequence of events in the dynamic event tree depends on the states of three underlying CD\&R functions: the strategic intent-based function (working or failed), the tactical intent-based function (working or failed), and the tactical state-based function (working or failed). Furthermore, since there are two aircraft in a collision pair, the state of each function on each aircraft must be considered. Table 13 shows sample results for the overtaking scenario. The first column lists the possible states of the tactical functions for a pair of aircraft. Specifically, $\mathrm{S}_{\mathrm{ijk}}$ denotes a state where $i(=0,1)$ strategic intentbased function are working, $j(=0,1,2)$ tactical intent-based functions are working and $k(=0,1,2)$ tactical state-based functions are working, as defined by the reliability diagrams in Figure 24, Figure 25 and Figure 26. For the strategic intent-based function, it is assumed that only one aircraft, namely the following aircraft, is responsible to execute a resolution to avoid the other aircraft. The second column shows the probability of observing the aircraft pair in this state. 
Table 13 Conditional collision probability of overtaking scenario due to pilot blunder

\begin{tabular}{|c|c|c|c|}
\hline$S_{i j k}$ & $\operatorname{Pr}\left\{S_{i j k}\right\}$ & $\operatorname{Pr}\left\{\right.$ collision |event $\left.1, S_{m n}\right\}$ & $\operatorname{Pr}\{$ collision|event 1$\}$ \\
\hline S122 & 9.99E-01 & $1.83 E-23$ & $1.83 E-23$ \\
\hline S121 & 1.94E-04 & 8.39E-21 & $1.63 E-24$ \\
\hline S120 & $9.40 \mathrm{E}-09$ & $1.08 \mathrm{E}-17$ & $1.02 E-25$ \\
\hline S112 & 2.34E-04 & $1.72 \mathrm{E}-21$ & $4.02 E-25$ \\
\hline S111 & $9.70 \mathrm{E}-05$ & $7.88 \mathrm{E}-19$ & $7.64 E-23$ \\
\hline S110 & 9.41E-09 & $1.01 \mathrm{E}-15$ & $9.55 \mathrm{E}-24$ \\
\hline S102 & 1.33E-08 & $3.22 \mathrm{E}-19$ & $4.28 E-27$ \\
\hline S101 & 9.41E-09 & 9.31E-17 & $8.76 E-25$ \\
\hline S100 & $9.13 E-13$ & $1.20 \mathrm{E}-13$ & $1.09 E-25$ \\
\hline S022 & 9.79E-05 & $1.68 \mathrm{E}-13$ & $1.65 E-17$ \\
\hline S021 & $1.90 \mathrm{E}-08$ & $7.72 \mathrm{E}-11$ & $1.47 E-18$ \\
\hline S020 & $9.22 \mathrm{E}-13$ & 9.94E-08 & $9.16 \mathrm{E}-20$ \\
\hline S012 & 4.00E-05 & $1.56 \mathrm{E}-11$ & $6.25 \mathrm{E}-16$ \\
\hline S011 & 3.61E-08 & 7.17E-09 & 2.59E-16 \\
\hline S010 & 9.41E-09 & 9.23E-06 & 8.69E-14 \\
\hline S002 & $9.70 \mathrm{E}-05$ & 2.93E-09 & $2.84 \mathrm{E}-13$ \\
\hline S001 & 1.72E-08 & 8.46E-07 & $1.45 \mathrm{E}-14$ \\
\hline SO00 & $1.04 \mathrm{E}-08$ & 2.54E-02 & 2.64E-10 \\
\hline sum & 1.00 & & 2.65E-10 \\
\hline
\end{tabular}

Figure 42 shows the state probabilities ordered from least likely to most likely.

The most likely state is the state in which all functions are working. This is expected

because a function failure requires multiple failures of components. The least likely state is the state in which the strategic intent-based function is working but both tactical intentbased functions and both tactical state-based functions are failed. 


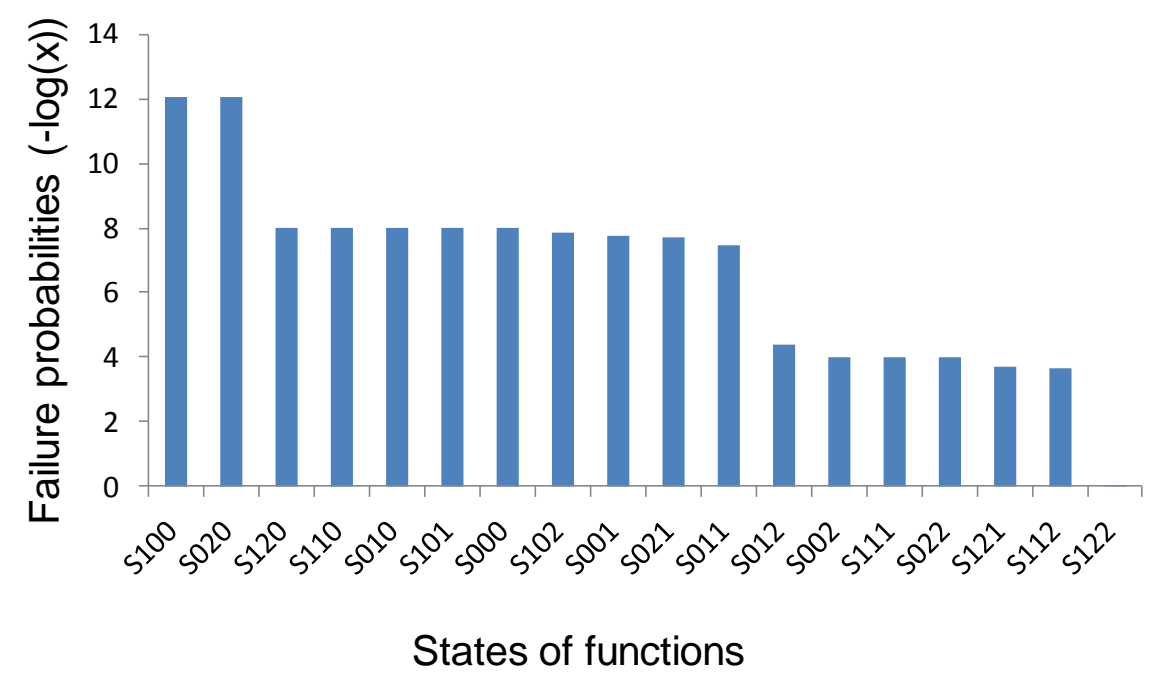

Figure 42 Failure probabilities of states of functions

The third column shows the conditional collision probability given the function states and pilot blunder resulting in an overtaking event. The last column is the conditional collision probability given the occurrence of the overtaking event (the product of columns 2 and 3). The safest case is when all functions are working. This case occurs $99.9 \%$ of time. The conditional collision probability is $1.83 \mathrm{E}-23$. The most dangerous case is when all functions fail. Column 4 also shows that this case dominates the conditional collision probability of the overtaking event even though it does not occur often. The results of state $S_{100}$ and $S_{022}$ suggest that one strategic intent-based function works as well as all tactical functions. This is because the strategic function has longer time to detect and resolve conflicts. Similarly, $S_{011}$ and $S_{002}$ suggest that one tactical intent-based function and one tactical state-based function works as well as 2 tactical state-based functions. 
Table 14 shows the results of the dynamic event tree for the lane change scenario. The structure of the table is the same as Table 13. The dynamic event tree of the lane change scenario contains tactical intent-based function and tactical state-based function.. The most likely state is when all functions are working $\left(S_{22}\right)$; the least likely state is when all functions are failed $\left(S_{00}\right)$. The third column gives the conditional collision probability given state $S_{i j}$ starting from the event where two aircraft are on course for an NMAC in the lane change scenario. Analogous tables are created for the three other collision scenarios as well (not shown). The conditional collision probabilities are evaluated using the dynamic event trees. The lowest collision probability occurs when all functions are working, the highest collision probability occurs when all functions are failed.

Table 14 Conditional collision probability of lane change scenario due to pilot blunder

\begin{tabular}{|l|c|c|c|}
\hline$S_{i j}$ & $\operatorname{Pr}\left\{S_{i j}\right\}$ & $\operatorname{Pr}\left\{\right.$ collision $\mid$ lane change, $\left.S_{i j}\right\}$ & $\operatorname{Pr}\{$ collision $\mid$ lane change $\}$ \\
\hline$S_{22}$ & $9.99 \mathrm{E}-01$ & $7.49 \mathrm{E}-15$ & $7.48 \mathrm{E}-15$ \\
\hline$S_{21}$ & $1.94 \mathrm{E}-04$ & $1.54 \mathrm{E}-12$ & $2.98 \mathrm{E}-16$ \\
\hline$S_{20}$ & $9.40 \mathrm{E}-09$ & $1.09 \mathrm{E}-09$ & $1.02 \mathrm{E}-17$ \\
\hline$S_{12}$ & $2.74 \mathrm{E}-04$ & $7.49 \mathrm{E}-15$ & $2.05 \mathrm{E}-18$ \\
\hline$S_{11}$ & $7.19 \mathrm{E}-08$ & $1.54 \mathrm{E}-12$ & $1.11 \mathrm{E}-19$ \\
\hline$S_{10}$ & $1.88 \mathrm{E}-08$ & $1.09 \mathrm{E}-09$ & $2.05 \mathrm{E}-17$ \\
\hline$S_{02}$ & $1.94 \mathrm{E}-04$ & $8.58 \mathrm{E}-09$ & $1.66 \mathrm{E}-12$ \\
\hline$S_{01}$ & $2.66 \mathrm{E}-08$ & $1.54 \mathrm{E}-06$ & $4.09 \mathrm{E}-14$ \\
\hline$S_{00}$ & $1.04 \mathrm{E}-08$ & $2.54 \mathrm{E}-02$ & $2.64 \mathrm{E}-10$ \\
\hline sum & 1.00 & & $2.66 \mathrm{E}-10$ \\
\hline
\end{tabular}

Table 15 shows the results of the dynamic event tree for the occurrence of simultaneous lane change event. The dynamic event tree for this scenario has only 3 conditions of the status of functions since it only includes the tactical state-based function. 
The most dangerous case is when all functions are working even though all functions failing dominate the third column. This is because $S_{0}$ occurs much less than $S_{2}$. The simultaneous lane change scenario has the highest conditional collision probability of all scenarios. This is because this scenario has the shortest time to an NMAC.

Table 15 Conditional collision probability of simultaneous lane change scenario due to pilot blunder

\begin{tabular}{|c|c|c|c|}
\hline$S_{\mathrm{i}}$ & $\operatorname{Pr}\left\{S_{i}\right\}$ & $\begin{array}{c}\operatorname{Pr}\{\text { collision } \mid \text { simultaneous lane } \\
\left.\text { change, } S_{i}\right\}\end{array}$ & $\begin{array}{c}\operatorname{Pr}\{\text { collision } \mid \text { simultaneous } \\
\text { lane change }\}\end{array}$ \\
\hline$S_{2}$ & 0.99961 & $1.09 \mathrm{E}-09$ & $1.09 \mathrm{E}-09$ \\
\hline$S_{1}$ & $3.88 \mathrm{E}-04$ & $1.09 \mathrm{E}-09$ & $4.22 \mathrm{E}-13$ \\
\hline$S_{0}$ & $3.86 \mathrm{E}-08$ & $2.54 \mathrm{E}-02$ & $9.81 \mathrm{E}-10$ \\
\hline sum & 1.00 & & $2.07 \mathrm{E}-09$ \\
\hline
\end{tabular}

Table 16 shows the results of dynamic event tree for the occurrence of the overtaking and lane change scenario. The dynamic event tree of this scenario has 18 possible function states, similar to the overtaking scenario. The conditional collision probability is slightly higher than the overtaking scenario but lower than the lane change scenario and the simultaneous lane change scenario. 
Table 16 Conditional collision probability of lane change and overtaking scenario due to pilot blunder

\begin{tabular}{|c|c|c|c|}
\hline$S_{i j k}$ & $\begin{array}{c}\operatorname{Pr}\left\{S_{i j k} \mid \text { lane change }\right. \\
\text { and overtaking }\}\end{array}$ & $\begin{array}{c}\operatorname{Pr}\{\text { collision } \mid \text { lane change and } \\
\left.\text { overtaking, } S_{i j k}\right\}\end{array}$ & $\begin{array}{c}\operatorname{Pr}\{\text { collision } \mid \text { lane } \\
\text { change and } \\
\text { overtaking }\}\end{array}$ \\
\hline$S_{122}$ & $9.99 \mathrm{E}-01$ & $4.65 \mathrm{E}-18$ & $4.64 \mathrm{E}-18$ \\
\hline$S_{121}$ & $1.94 \mathrm{E}-04$ & $2.06 \mathrm{E}-15$ & $3.98 \mathrm{E}-19$ \\
\hline$S_{120}$ & $9.40 \mathrm{E}-09$ & $2.51 \mathrm{E}-12$ & $2.36 \mathrm{E}-20$ \\
\hline$S_{112}$ & $2.34 \mathrm{E}-04$ & $5.66 \mathrm{E}-17$ & $1.32 \mathrm{E}-20$ \\
\hline$S_{111}$ & $9.70 \mathrm{E}-05$ & $2.50 \mathrm{E}-14$ & $2.43 \mathrm{E}-18$ \\
\hline$S_{110}$ & $9.41 \mathrm{E}-09$ & $3.06 \mathrm{E}-11$ & $2.88 \mathrm{E}-19$ \\
\hline$S_{102}$ & $1.33 \mathrm{E}-08$ & $4.77 \mathrm{E}-15$ & $6.34 \mathrm{E}-23$ \\
\hline$S_{101}$ & $9.41 \mathrm{E}-09$ & $1.37 \mathrm{E}-12$ & $1.29 \mathrm{E}-20$ \\
\hline$S_{100}$ & $9.13 \mathrm{E}-13$ & $1.67 \mathrm{E}-09$ & $1.53 \mathrm{E}-21$ \\
\hline$S_{022}$ & $9.79 \mathrm{E}-05$ & $3.02 \mathrm{E}-12$ & $2.96 \mathrm{E}-16$ \\
\hline$S_{021}$ & $1.90 \mathrm{E}-08$ & $1.34 \mathrm{E}-09$ & $2.54 \mathrm{E}-17$ \\
\hline$S_{020}$ & $9.22 \mathrm{E}-13$ & $1.63 \mathrm{E}-06$ & $1.50 \mathrm{E}-18$ \\
\hline$S_{012}$ & $4.00 \mathrm{E}-05$ & $3.68 \mathrm{E}-11$ & $1.47 \mathrm{E}-15$ \\
\hline$S_{011}$ & $3.61 \mathrm{E}-08$ & $1.63 \mathrm{E}-08$ & $5.87 \mathrm{E}-16$ \\
\hline$S_{010}$ & $9.41 \mathrm{E}-09$ & $1.99 \mathrm{E}-05$ & $1.87 \mathrm{E}-13$ \\
\hline$S_{002}$ & $9.70 \mathrm{E}-05$ & $3.10 \mathrm{E}-09$ & $3.01 \mathrm{E}-13$ \\
\hline$S_{001}$ & $1.72 \mathrm{E}-08$ & $8.93 \mathrm{E}-07$ & $1.53 \mathrm{E}-14$ \\
\hline$S_{000}$ & $1.04 \mathrm{E}-08$ & $2.54 \mathrm{E}-02$ & $2.64 \mathrm{E}-10$ \\
\hline$s_{1}$ & 1.00 & & $2.65 \mathrm{E}-10$ \\
\hline & & & \\
\hline
\end{tabular}

A sensitivity analysis is conducted for both case studies. The analysis varies one parameter at a time (increasing the value by 10 times) and computes the ratio of the resulting collision probability divided by the baseline value. A value of 1.0 means that the parameter change does not affect the final collision probability, so the parameter is not critical. Parameters to vary include the transition probabilities in the dynamic event tree and the failure probabilities of the components. For example, for case study 1 (loss of locatability), the parameters that can be varied are 1) failure probabilities of components in the TCAS function, 2) the probability that both pilots fail to respond to TCAS, and 3) 
the probability of a collision occurring by chance given an NMAC. Figure 43 shows that the last two parameters for case study 1 are important.

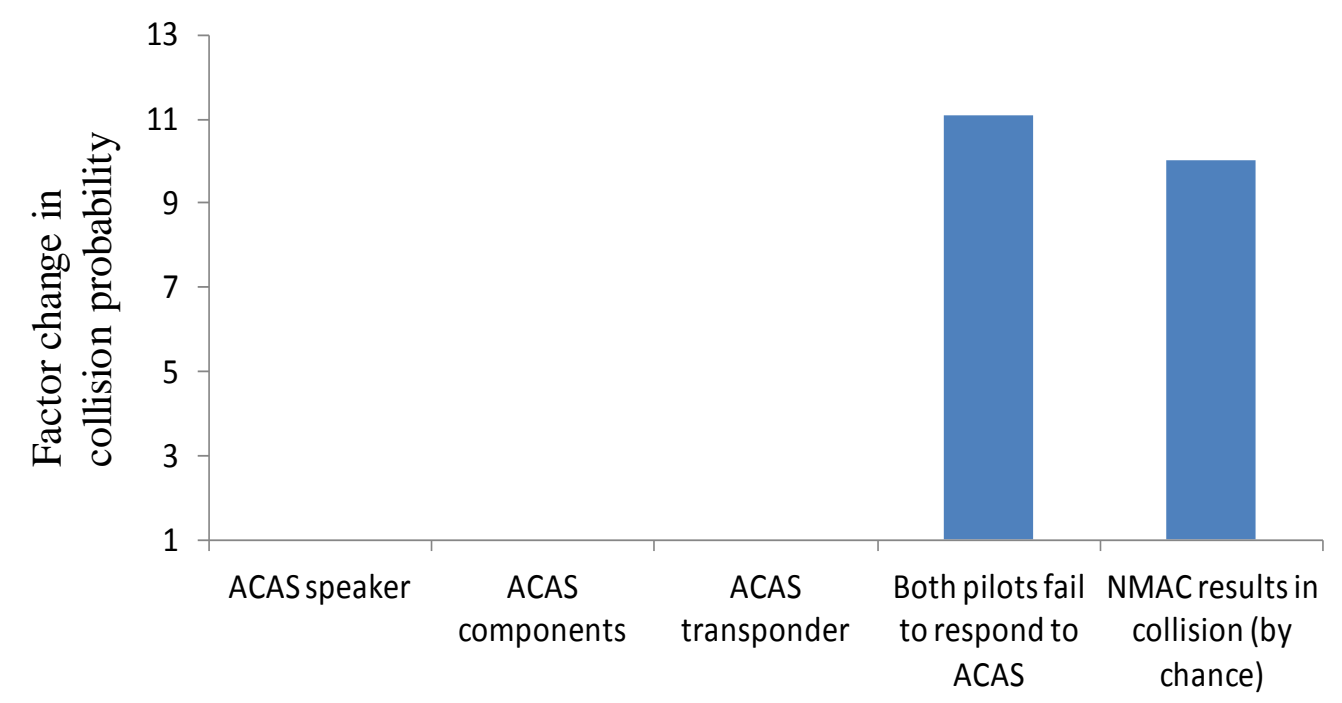

Figure 43 Sensitivity analysis of collision scenarios due to loss of locatability

For the pilot blunder case, Figure 44 shows the sensitivity analysis for overtaking event. The results show that the two parameters discussed above in case study 1 are also the most critical ones here. The ADS-B in transponder failure probability is a third critical parameter that affects the final collision probability. 


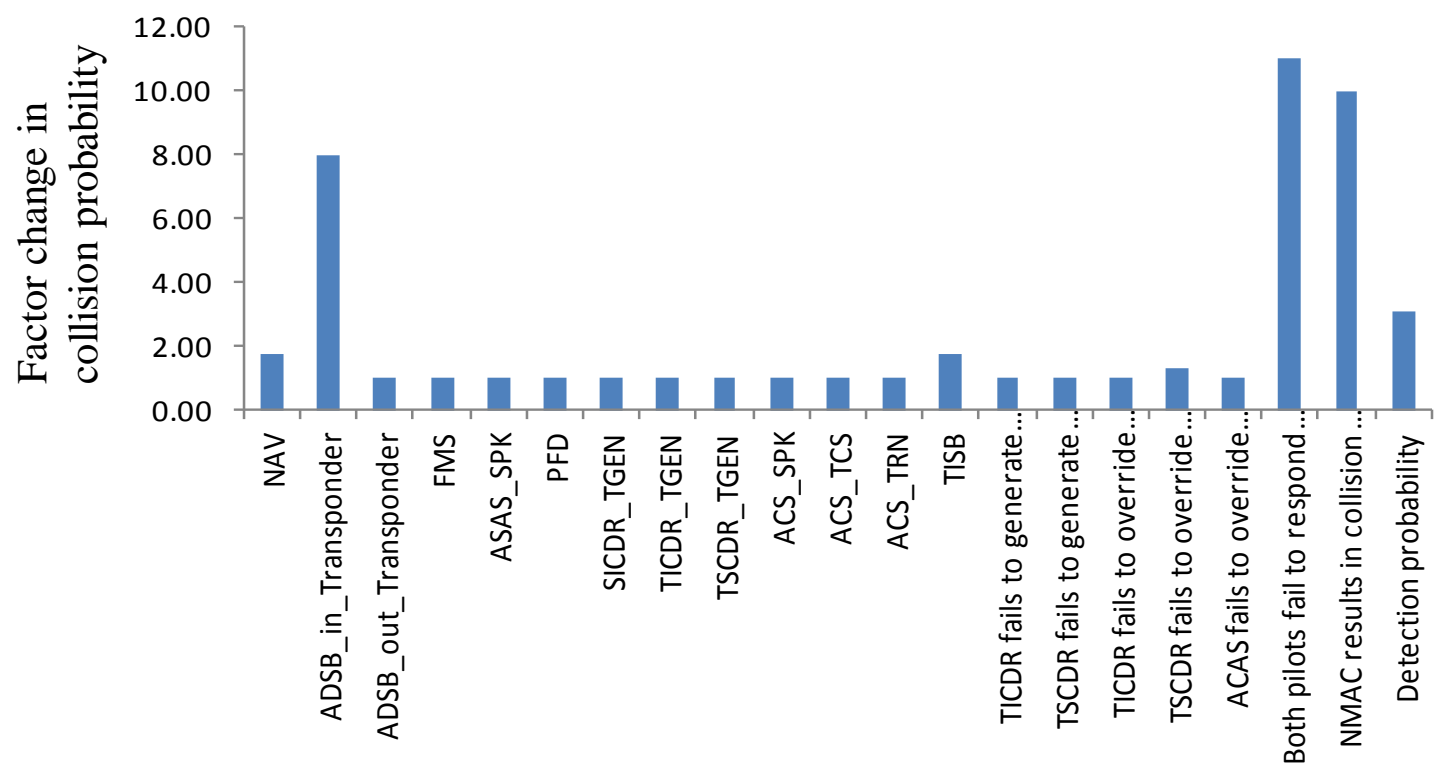

Figure 44 Sensitivity analysis of the overtaking scenario

The sensitivity analysis of lane change event has similar results as shown in Figure 45. However, the detection probability is not as important as it is in overtaking event. The reason is that when two aircraft are close to the NMAC, it is much easier to detect the conflict. The results for simultaneous lane change event in Figure 46 show that the detection probability is not a critical parameter when aircraft are closer to the NMAC. The trajectory generation is important because the key point at this time frame is to generate the resolution and prevent the collision. 


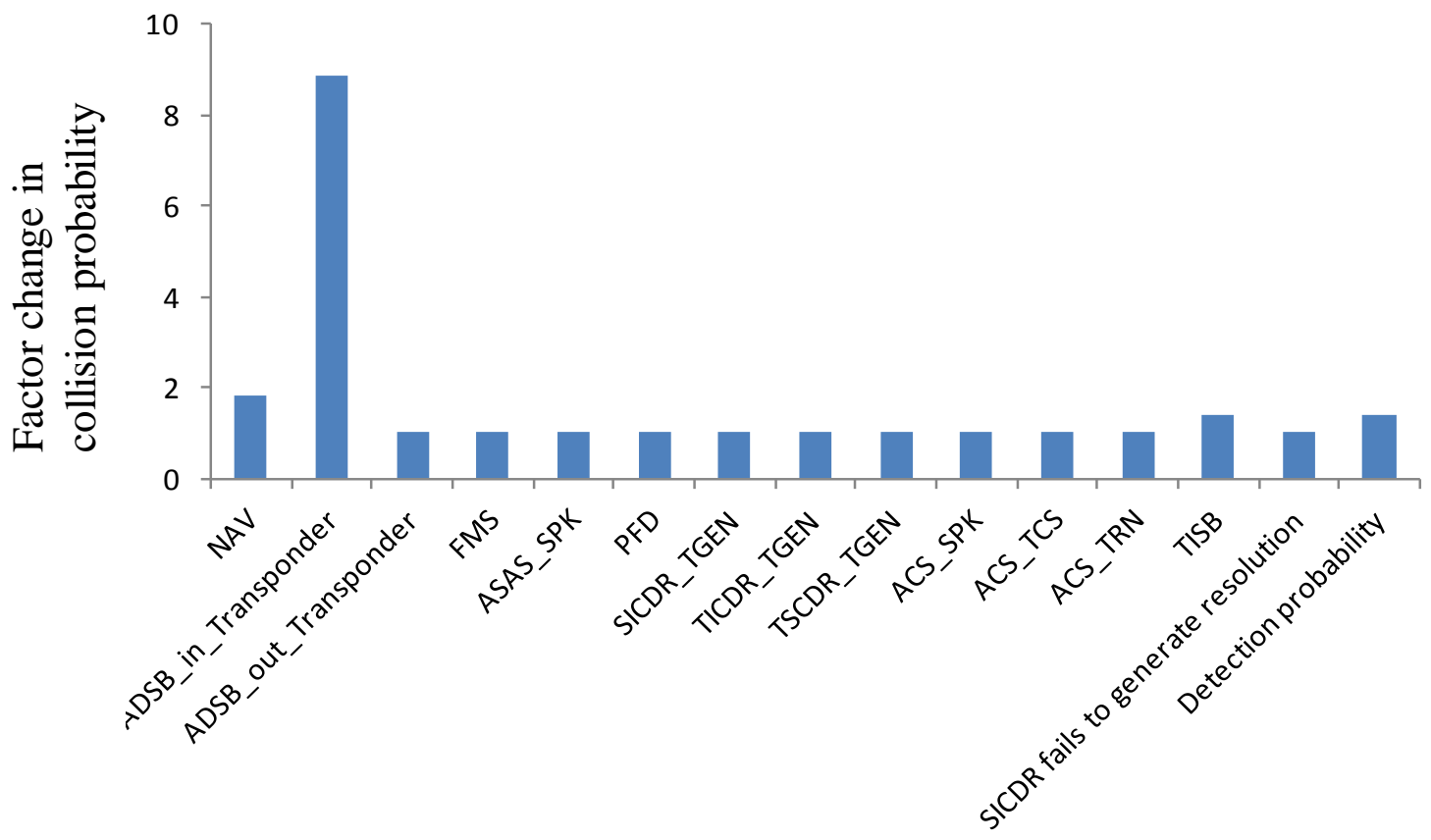

Figure 45 Sensitivity analysis of lane change scenario

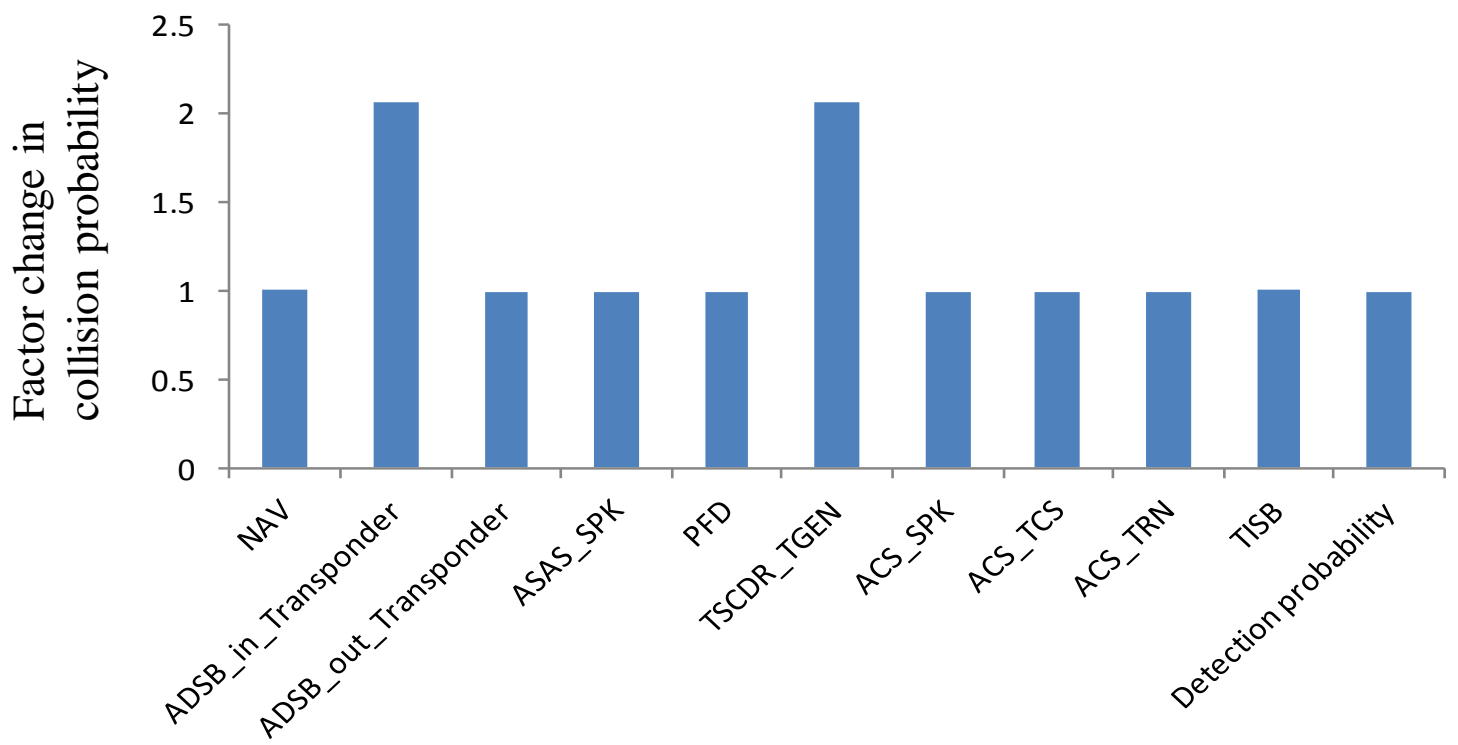

Figure 46 Sensitivity analysis of simultaneous lane change scenario 
The results of sensitivity analysis of event 4 are also similar with that of event 1 .

The impact of detection probability has slightly difference.

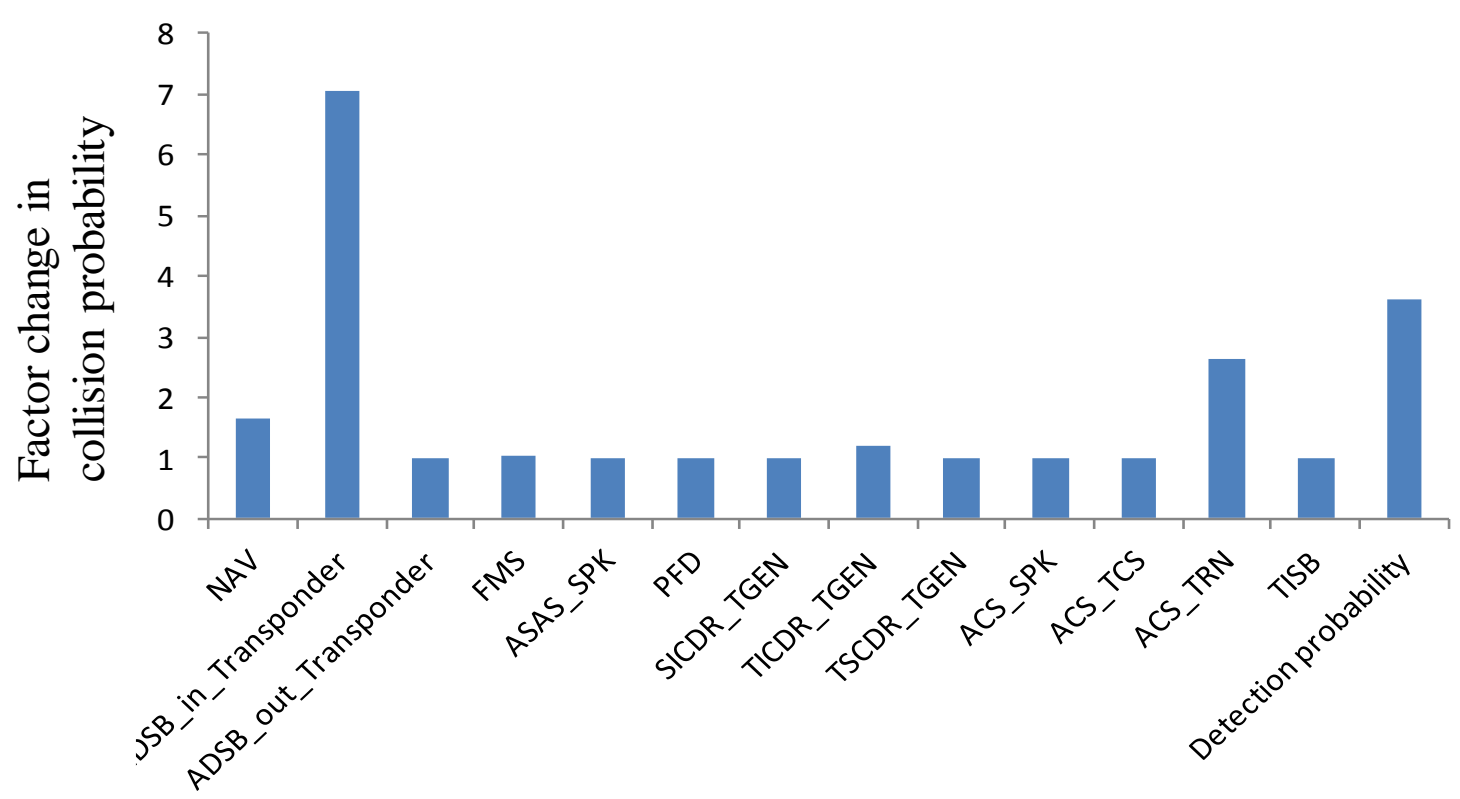

Figure 47 Sensitivity analysis of lane change and overtaking scenario

\subsection{Results of the Case Studies}

Table 17 shows the overall collision probability due to loss of locatability. The collision probability for each event is the produce of columns 2,3 and 4 . The occurrence rates of potential NMAC trajectories are using a minimum separation of 3 nautical miles. All other parameters are set to their baseline values. The probability of losing the locatability function is calculated with a reliability diagram. The conditional collision probability is calculated using the dynamic event tree when only TCAS works. The overall collision probability due to loss of locatability satisfies the current level of safety. 
Table 17 Collision probability due to loss of locatability

\begin{tabular}{|c|c|c|c|c|}
\hline & $\begin{array}{c}\operatorname{Pr}\{\text { potential } \\
\text { NT }\}\end{array}$ & \begin{tabular}{|c|}
$\operatorname{Pr}\{$ LOL $\mid$ potential \\
$\mathrm{NT}\}$
\end{tabular} & $\begin{array}{c}\operatorname{Pr}\{\text { collision } \mid \text { potential } \\
\text { NT, LOL }\}\end{array}$ & $\operatorname{Pr}\{$ collision $\}$ \\
\hline 0vertaking & $1.44 \mathrm{E}-01$ & $2.32 \mathrm{E}-07$ & $2.54 \mathrm{E}-02$ & $8.51 \mathrm{E}-10$ \\
\hline Lane change & $3.81 \mathrm{E}-02$ & $2.32 \mathrm{E}-07$ & $2.54 \mathrm{E}-02$ & $2.24 \mathrm{E}-10$ \\
\hline $\begin{array}{c}\text { Simultaneous lane } \\
\text { change }\end{array}$ & 1. $49 \mathrm{E}-02$ & 2. $32 \mathrm{E}-07$ & 7. $02 \mathrm{E}-02$ & 2. $43 \mathrm{E}-10$ \\
\hline $\begin{array}{c}\text { Lane change and } \\
\text { overtaking }\end{array}$ & 8. $12 \mathrm{E}-02$ & 2. $32 \mathrm{E}-07$ & 2. $54 \mathrm{E}-02$ & 4. $79 \mathrm{E}-10$ \\
\hline sum & & & & 1. $80 \mathrm{E}-09$ \\
\hline
\end{tabular}

The collision probability of the second case study is shown in Table 18 . The probability of the potential NMAC trajectory from the simulation uses the same values as the loss of locatability case. The probability of the operational error is assumed to be 1.5E-05. Simultaneous lane change scenario is the most dangerous case. However, it occurs less frequently that other events. The overtaking scenario dominates the overall collision probability. The overall collision probability due to pilot blunder also satisfies the current level of safety.

Table 18 Collision probability duel to pilot blunders

\begin{tabular}{|c|c|c|c|c|}
\hline & $\begin{array}{c}\operatorname{Pr}\{\text { potential } \\
\text { NT }\}\end{array}$ & $\operatorname{Pr}\{\mathrm{PD} \mid \mathrm{NT}\}$ & $\begin{array}{c}\operatorname{Pr}\{\text { col1ision } \mid \text { potential } \\
\text { NT, PD }\}\end{array}$ & $\operatorname{Pr}\{$ collision $\}$ \\
\hline 0vertaking & $1.44 \mathrm{E}-01$ & $1.50 \mathrm{E}-05$ & $2.65 \mathrm{E}-10$ & $5.74 \mathrm{E}-16$ \\
\hline Lane change & $3.81 \mathrm{E}-02$ & $1.50 \mathrm{E}-05$ & $2.66 \mathrm{E}-10$ & $1.52 \mathrm{E}-16$ \\
\hline $\begin{array}{c}\text { Simultaneous lane } \\
\text { change }\end{array}$ & $1.49 \mathrm{E}-02$ & $1.50 \mathrm{E}-05$ & $2.07 \mathrm{E}-09$ & $4.63 \mathrm{E}-16$ \\
\hline $\begin{array}{c}\text { Lane change and } \\
\text { overtaking }\end{array}$ & $8.12 \mathrm{E}-02$ & $1.50 \mathrm{E}-05$ & $2.65 \mathrm{E}-10$ & $3.23 \mathrm{E}-16$ \\
\hline sum & & & & $1.51 \mathrm{E}-15$ \\
\hline
\end{tabular}




\subsection{Trade-off between Throughput and Collision Probability}

A trade-off curve is established between throughput and collision probability.

This curve is constructed using the baseline simulation experiment (Section 6.1) and the loss-of-locatability case study, varying failure probabilities of components in the reliability diagram and transition probabilities in the dynamic event tree. The loss-oflocatability case is much more dangerous than the pilot-blunder case and dominates the overall collision probability of the flow corridor concept-of-operations. Figure 48 shows that the flow corridor satisfies the target level of safety (5E-9) and throughput can be safely increased to 127 aircraft per hour by reducing the minimum separation to 2 nautical miles. Given the fixed width and lane-change angle of a flow corridor, the collision probability of the lane change scenario does not change much as the minimum separation reduces. The increased overall collision probability is primarily due to the occurrence rate of the overtaking scenario. The error bars represent the $95 \%$ of confidence interval which considers simulation variability, and parameter variability. The limitations of this model are exclusions of wake vortex issues and secondary conflicts. When the minimum separation is reduced to $2 \mathrm{~nm}$, such problems become important to the system. 


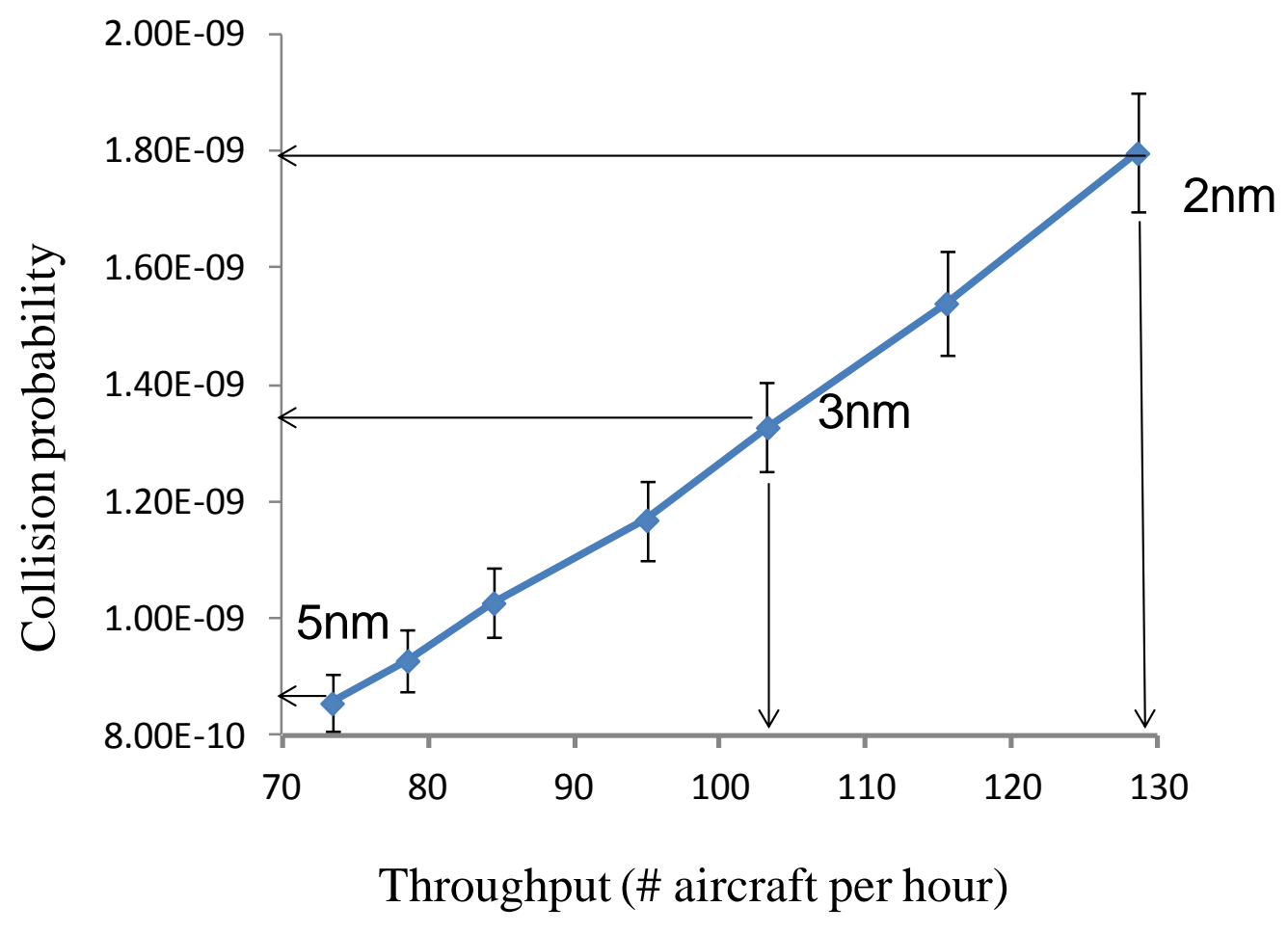

Figure 48 Trade-off between throughput and collision probability

\subsection{Summary}

This chapter discussed the combined results of the Monte Carlo simulation and the dynamic event tree analysis. The overall collision probabilities were calculated using Equation 3. Both case studies satisfy the current level of safety, though the loss-oflocatability case is less safe than the pilot blunder case. The trade-off curve suggests that throughput can be safely increased by reducing separation to 2 nautical miles. However, in regards to the sensitivity analysis, critical parameters are the probability that both pilots fail to respond to TCAS, the probability that an NMAC results in a collision by chance, and the failure probability of the ADS-B in transponder. For example, if the 
failure probability of the ADS-B in transponder increases by a factor of 10 , the overall collision probability violates the $1 \mathrm{E}-9$ level of safety. 


\section{CHAPTER 7: CONCLUSIONS AND RECOMMENDATIONS FOR FUTURE RESEARCH}

\subsection{Conclusions}

The dissertation developed a methodology to estimate the collision probability of the flow corridor concept-of-operations. The methodology decomposes the collision probability into three parts based on the sequence of events leading to a collision. First, a Monte Carlo simulation of the flow corridor dynamics provides the occurrence rates of potential NMAC trajectories. Potential NMAC trajectories occur during normal operations at the decision points where an erroneous decision would lead to two aircraft being on course for an NMAC trajectory. Second, reliability diagrams are used to model the causes that a potential NMAC trajectory becomes an actual NMAC trajectory. These causes include equipment failures leading to a loss of locatability and pilot blunders. Third, dynamic event trees are used to determine the probability of a collision given that two aircraft are on course for actual NMAC trajectory. The dynamic event trees model the onboard conflict detection and resolution functions that provide safety layers to prevent a collision. This dissertation considers the ADS-B system, lane change maneuvers, and the automated separation assurance system which are the main characteristics of the flow corridor concept. 


\subsection{Level of Safety}

This dissertation analyzes a 2-lane flow corridor. The overall collision probability of the 2-lane flow corridor meets the target level of safety. This considers four collision scenarios and event sequences leading to a collision.

\subsection{Throughput}

Throughput can be safely increased to 127 aircraft per hour by reducing the minimum separation to 2 nautical miles. The collision probability still meets the target level of safety.

\subsection{Key Safety Vulnerabilities}

A sensitivity analysis shows that the most critical parameters in the model related to the overall collision probability are the minimum separation, the probability that both flights fail to respond to TCAS, the probability that an NMAC results in collision by chance, the failure probability of the ADS-B in transponder, and the conflict detection probability.

\subsection{Future Work}

\subsubsection{Improved Analysis}

This dissertation can be further improved by extending the analysis to multi-lane flow corridors. For example a $2 \times 2$ flow corridor can include vertical lane change maneuvers.

\subsubsection{Improved Modeling}

This dissertation can be further improved with refined modeling by considering all collision scenarios, secondary conflicts and wake vortex encounters. This dissertation assumes non-flow corridor cannot penetrate the corridor traffic flow. However, a 
collision can be caused by an intruder aircraft. Secondary conflicts and wake vortices model can also be considered when the minimum separation is small. For example, a secondary conflict can increase collision probability. Wake vortex constraints were not considered in this dissertation, and they can be a limitation to reduce the minimum separation. 


\section{APPENDIX}

Table 19 Reliability diagram parameters

\begin{tabular}{|c|c|c|c|}
\hline Components & Failure probability & Baseline values & Reference \\
\hline Navigation, aircraft \#1 & $\mathrm{P}_{1}$ & 0.0005 & {$[\mathrm{Hemm}, 2009]$} \\
\hline $\begin{array}{l}\text { ADS-B in receiver, } \\
\text { aircraft \#1 }\end{array}$ & $\mathrm{P}_{2}$ & 0.000097 & {$[\mathrm{Hemm}, 2009]$} \\
\hline Navigation, aircraft \#2 & $\mathrm{P}_{3}$ & 0.0005 & [Hemm,2009] \\
\hline $\begin{array}{c}\text { ADS-B out } \\
\text { transmitter, aircraft \#2 }\end{array}$ & $\mathrm{P}_{4}$ & 0.000097 & [Hemm,2009] \\
\hline TIS-B & $\mathrm{P}_{5}$ & 0.000001 & Assumed \\
\hline FMS, aircraft \#1 & $\mathrm{P}_{6}$ & 0.00004 & [Blum,2010] \\
\hline $\begin{array}{l}\text { SICDR trajectory } \\
\text { generator, aircraft } 1\end{array}$ & $\mathrm{P}_{7}$ & 0.000001 & Assumed \\
\hline $\begin{array}{c}\text { Navigation display, } \\
\text { aircraft \#1 }\end{array}$ & $\mathrm{P}_{8}$ & 0.000001 & Assumed \\
\hline $\begin{array}{l}\text { TICDR trajectory } \\
\text { generator, aircraft } 1\end{array}$ & $\mathrm{P}_{9}$ & 0.000001 & Assumed \\
\hline $\begin{array}{l}\text { Primary flight display, } \\
\text { aircraft \#1 }\end{array}$ & $\mathrm{P}_{10}$ & 0.000001 & Assumed \\
\hline $\begin{array}{l}\text { ASAS speaker, } \\
\text { aircraft \#1 }\end{array}$ & $\mathrm{P}_{11}$ & 0.00055 & [Blum,2010] \\
\hline $\begin{array}{l}\text { TSCDR trajectory } \\
\text { generator, aircraft } 1\end{array}$ & $\mathrm{P}_{12}$ & 0.000001 & Assumed \\
\hline $\begin{array}{l}\text { TCAS transponder, } \\
\text { aircraft \#1 }\end{array}$ & $\mathrm{P}_{13}$ & 0.000097 & [Hemm,2009] \\
\hline $\begin{array}{l}\text { TCAS speaker, } \\
\text { aircraft \#1 }\end{array}$ & $\mathrm{P}_{14}$ & 0.000001 & {$[\mathrm{Hemm}, 2009]$} \\
\hline $\begin{array}{c}\text { TCAS other } \\
\text { components, aircraft } \\
\# 1\end{array}$ & $\mathrm{P}_{15}$ & 0.000292 & [Hemm,2009] \\
\hline $\begin{array}{l}\text { TCAS transponder, } \\
\text { aircraft } \# 2\end{array}$ & $\mathrm{P}_{16}$ & 0.000097 & [Hemm,2009] \\
\hline $\begin{array}{l}\text { TCAS speaker, } \\
\text { aircraft } \# 2\end{array}$ & $\mathrm{P}_{17}$ & 0.000001 & [Hemm,2009] \\
\hline $\begin{array}{c}\text { TCAS other } \\
\text { components, aircraft }\end{array}$ & $\mathrm{P}_{18}$ & 0.000292 & [Hemm,2009] \\
\hline
\end{tabular}




\section{\#2}

Table 20 Dynamic event tree parameters

\begin{tabular}{|c|c|c|c|}
\hline Components & Failure probability & Baseline values & Reference \\
\hline $\begin{array}{c}\text { Conflict detection } \\
\text { generate resolution }\end{array}$ & $\mathrm{R}_{1}$ & 0.000001 & [Ezberger,1997] \\
\hline $\begin{array}{c}\text { TICDR fails to } \\
\text { generate resolution }\end{array}$ & $\mathrm{R}_{2}$ & 0.000001 & Assumed \\
\hline $\begin{array}{c}\text { TSCDR fails to } \\
\text { generate resolution }\end{array}$ & $\mathrm{R}_{3}$ & 0.000001 & Assumed \\
\hline $\begin{array}{c}\text { TICDR fails to } \\
\text { override SICDR }\end{array}$ & $\mathrm{R}_{4}$ & 0.000001 & Assumed \\
\hline $\begin{array}{c}\text { TSCDR fails to } \\
\text { override TICDR }\end{array}$ & $\mathrm{R}_{5}$ & 0.000001 & Assumed \\
\hline $\begin{array}{c}\text { TCAS fails to } \\
\text { override TSCDR }\end{array}$ & $\mathrm{R}_{6}$ & 0.000001 & Assumed \\
\hline $\begin{array}{c}\text { Both pilots fail to } \\
\text { respond to TCAS }\end{array}$ & $\mathrm{R}_{7}$ & 0.3 & Assumed \\
\hline $\begin{array}{c}\text { NMAC results in } \\
\text { collision (by chance) }\end{array}$ & $\mathrm{R}_{8}$ & 0.12 & [Blum,2010] \\
\hline $\begin{array}{c}\text { Pilot fails to accept } \\
\text { resolution in .5 min }\end{array}$ & $\mathrm{R}_{9}$ & 0.1 & [Consiglio,2010] \\
\hline $\begin{array}{c}\text { Pilot fails to accept } \\
\text { TICDR resolution in } \\
\text { 20 seconds }\end{array}$ & $\mathrm{R}_{10}$ & 0.2 & \\
\hline $\begin{array}{c}\text { Pilot fails to accept } \\
\text { TSCDR resolution in } \\
10 \text { seconds }\end{array}$ & $\mathrm{R}_{11}$ & $\mathrm{R}_{12}$ & 0.3 \\
\hline
\end{tabular}




\section{REFERENCES}

Alipio, J., Castro, P., Kaing, H., Shahd, N., Sheizai, O., Donohue, G., and Grundmann, K. (October 2003) Dynamic Airspace Super Sectors (DASS) As High-Density Highways in The Sky for A New US Air Traffic Management System. AIAA/IEEE Digital Avionics Systems Conference.

Amalberti, R. (1998) Automation in aviation: A human factors perspective. Aviation human factors, 173-192.

Bakker, G.J., Blom H. (1993) Air Traffic Collision Risk Modelling. In $32^{\text {nd }}$ IEEE Conference on Decision and Control , 1993, pp. 1404-1409.

Belle, A. and Yousefi, A. (2010) Analysis of Performance of Q Routes for establishing Future Design Criteria. Integrated Communications Navigation and Surveillance (ICNS) Conference, Virgina.

Belle, A., Shortle J., Yousefi A. (2012) Estimation of potential conflict rates as a function of sector loading. Proceedings of the International Conference on Research in Air Transportation, Berkeley, CA.

Belle, A., Shortle J. (2013) A methodology for estimating probability of secondary conflict due to simultaneous resolution. 32nd Digital Avionics Systems Conference. Syracuse, NY.

Blom, H. A.P., Bakker, G.J., Krystul, J., Everdij, M.H.C., Obbink B., Klompstra M.B. (2005) Sequential Monte Carlo simulation of collision risk in free flight air traffic. HYBRIDGE D9.4

Blum, D, Thipphavong, D., Rentas, T., He, Y., Wang, X., (2010) Safety analysis of the automated airspace concept using Monte Carlo simulation, AIAA Guidance, Navigation, and Control Conference, Aug. 2-5

Boeing. (2008) Summary of Commercial Jet Airplane Accidents. Worldwide Operations, 1959-2007.

Campos, L.M.B.C., (2001), On the probability of Collision Between Aircraft with DissimilarPosition Errors, Journal of Aircraft, 38, pp. 593-599. 
Campos, L.M.B.C. \& Marques, J.M.G., (2002), On Safety Metrics Related to Aircraft Separation, Journal of Navigation, Vol. 55, pp. 39-63.

Consiglio, M., Hoadley, S., Wing D., and Baxley B. (2007) Safety performance of airborne separation: preliminary baseline testing. 7th AIAA Aviation Technology, Integration and Operations Conference (ATIO), Belfast, Northern Ireland.

Campos, L.M.B.C. \& Marques, J.M.G., (2011) On the Probability of Collision for Crossing, Aircraft Engineering and Aerospace Technology: An International Journal, Volume 83, Number 5, 2011 , pp. 306-314(9)

Daams, J., Bakker, G.J., Blom, H.A.P. (1999). Safety evaluation of encounters between free flight equipped aircraft in a dual route structure, NLR-TR-99577

Department of Research and Definition of Air Navigation Advanced Systems Navigation and Surveillance Division (2012) EUR/SAM Corridor:2010 Collision Risk Assessment. NYVI-IDSA-INF-003-1.0/12

Erzberger, H., Paielli, R., Isaacson D., Eshowl M. (1997) Conflict detection and resolution in the presence of prediction error. 1st USA/Europe Air Traffic Management R\&D Seminar, Saclay, France.

Erzberger, H. (2001) The automated airspace concept. 4th USA/Europe Air Traffic management R\&D Seminar, Santa Fe, NM.

Erzberger, H. (2006) Automated conflict resolution for air traffic control. $25^{\text {th }}$ International congress of the aeronautical sciences.

Erzberger, H., Heere, K. (2011) Algorithm and operational concept for resolving shortrange conflicts. Proceedings of the Institution of Mechanical Engineers, Part G: Journal of Aerospace Engineering.

FAA aerospace forecasts fiscal years $2010-2033$

FAA report, National Airspace System (NAS) En Route Airspace Assessment, 2007.

Gupta, G., Sridhar, B., and Mukherjee, A. (2008) Freeways in the Sky: Exploring Tube Airspace design through Mixed Integer Programming INFORMS Annual Meeting, Washington, D.C.

Hemm, R. and A. Busick, (2009) Safety analysis of the separation assurance function in today's national airspace system, LMI Research Institute, report NS801T2. 
Hofer E., Kloos M., Krzykacz-Hausmann B., Peschke J. and Sonnenkalb N. (2004) Dynamic Event Trees for Probabilistic Safety Analysis. GRS

Hoffman, Robert, Joseph P. (2008) Principles of Airspace Tube Design for Dynamic Airspace Configuration. Anchorage, Alaska, AIAA-ATIO Conference

Joint Planning and Development Office (June 2012) Concept of Operations for the Next Generation Air Transportation System. Version 3.2.

Kopardekar, P., Bilimoria, K., and Sridhar, B. (2007) Initial Concepts for Dynamic Airspace Configuration. 7th AIAA Aviation Technology, Integration and Operations Conference (ATIO), Belfast, Northern Ireland.

Kotecha, P. and Hwang, I. (2009) Optimization based Tube Network Design for the Next Generation Air Transportation System (NEXTGEN). AIAA Guidance, Navigation, and Control Conference and Exhibit, Chicago, IL.

Kuchar J. and Yang L. (2000) A Review of Conflict Detection and Resolution Modeling Methods. IEEE Transactions on Intelligent Transportation Systems, Vol. 1, No. 4, pp. 179-189.

Kuchar J., Andrews J., Drumm A. et al. (2004) A safety analysis process for the traffic alert and collision avoidance system (TCAS) and see-and-avoid systems on remotely piloted vehicles. AIAA 3rd "Unmanned Unlimited Technical Conference, Chicago.

Lebron, J., et al. (1983) System Safety of Minimum TCAS II. The MITRE Corporation. MTR 83W241

Moek, G. and Harrison D., (1992) European Studies to Investigate the Feasibility of Using 1000 ft Vertical Separation Minima Above FL290, Part II, Precision Radar Data Analysis and Collision Risk Assessment, Journal of Navigation, Vol.45, no 1, 1992, pp 91-106.

Mundra A. and Simmons E. (2007) Self-Separation Corridors. Digital Avionics Systems Conference, Dallas, TX.

North Atlantic systems planning group (2013) ICAO European and North Atlantic Office

Reich, P.G., (1966), Analysis of Long-range Air Traffic Systems: Separation Standards. Journal of Navigation, pp. 88-98, pp. 169-186, pp. 331-347. 
Shortle, J.F., Xie, Y., Chen, C.H. \& Dunohue, G.L. (2004) Simulating Collision Probabilities of Landing Airplanes at Non-towered Airports. Simulation, pp. 2131.

Shortle, J., Sherry L., Yousefi A., Xie R. (2012) Safety and sensitivity analysis of the advanced airspace concept for NextGen. Proceedings of the Integrated Communication, Navigation, and Surveillance Conference, Herndon, VA.

Shortle, J., Zhang Y. (2013) Safety comparison of centralized and distributed automated separation concepts. IEEE Transactions on Reliability, Vol. 63, issue 1.

Siddiqee W. (1973) A mathematical model for predicting the number of potential conflict situations. Transportation Science, vol. 7 no. 2 158-167

Sridhar, B., Grabbe, S., Sheth, K., and Bilimoria, K. (2006) Initial Study of Tube Networks for Flexible Airspace Utilization. AIAA Guidance, Navigation, and Control Conference and Exhibit, Keystone, Colorado.

Takeichi N., Nakamura Y. and Fukuoka K. (2012) Fundamental characteristics of decentralized air traffic flow control in high density corridor. 28th Congress of the International Council of the Aeronautical Sciences, Australia.

Watson H. A., Hill M. (1961) Launch Control Safety Study. Section VII, Vol 1 Bell Labs.

Wing, D., and Ballin M. (2004) Pilot in command: A feasibility assessment of autonomous flight management operations. 24th International Congress of the Aeronautical Sciences.

Wing, D. J., Smith, J. C., and Ballin, M. G. (2008) Analysis of a Dynamic Multi-Track Airway Concept for Air Traffic Management. Tech. Rep. NASA/TP-2008-215323, Langley Research Center, Hampton, Virginia.

Wing, D., Murdoch J., Chamberlain J., Consiglio M., Hoadley S., Hubbs C., and Palmer M. (2010) Function allocation with airborne self-separation evaluated in a piloted simulation. NASA Langley Research Center Technical Report NF1676L9177.

Wing, D.J. and Cotton, W.B., (2011) Autonomous Flight Rules: A Concept for Self Separation in US Domestic Airspace. NASA Technical Paper

Xue, M. and Kopardekar, P. (2009) High-Capacity Tube Network Design using the Hough Transform. Journal of Guidance, Control, and Dynamics, Vol. 32, No. 3, pp. 788-795 
Xue, M. (2009) Design Analysis of Corridors-in-the-sky. AIAA Guidance, Navigation, and Control Conference and Exhibit, Chicago, IL.

Ye, B., Hu M., Shortle J. (2014) Collision risk-capacity tradeoff analysis of an en-route corridor model. Chinese Journal of Aeronautics, Vol. 27 pp 124-135.

Yousefi, A., Donohue, G., and Sherry, L. (2004) High-Volume Tube-Shape Sectors(HTS): A Network of High Capacity Ribbons Connecting Congested City Pairs. Proceedings of the 23rd Digital Avionics Systems Conference, Salt Lake City, CT.

Yousefi, A., John T., Ben S., and Frederick J. (2009) Operational Procedures for the Corridors-in-the-Sky. Dulles, VA, Technical Report NNA08BA50D to NASA Ames, Metron Aviation Inc.

Yousefi, A., Zadeh A., and Tafazzoli, A. (2010), Dynamic Allocation and Benefit Assessment of NextGen Flow Corridors. Fort Worth, Texas, In Proceedings of 10th AIAA-ATIO Conference.

Yousefi, A., Lard J., Timmerman J. (2010) NextGen flow corridors initial design, procedures, and display functionalities. 29th Digital Avionics Systems Conference

Yousefi, A., Xie R., Krishna S., Shortle J., Zhang Y. (2012). Safety analysis tool for automated airspace concepts (SafeATAC). Proceedings of the 31st Digital Avionics Systems Conference, Williamsburg, VA, 4C2-1 - 4C2-13.

Zhang, Y., Shortle J., Sherry L. (2013) Methodology for collision risk assessment of flow corridor concept. Proceedings of the Integrated Communication, Navigation, and Surveillance Conference, Herndon. VA. 


\section{BIOGRAPHY}

Yimin Zhang graduated from Harbin Engineering University, China, in 2007. He received his Master degree from George Mason University in 2009. 